
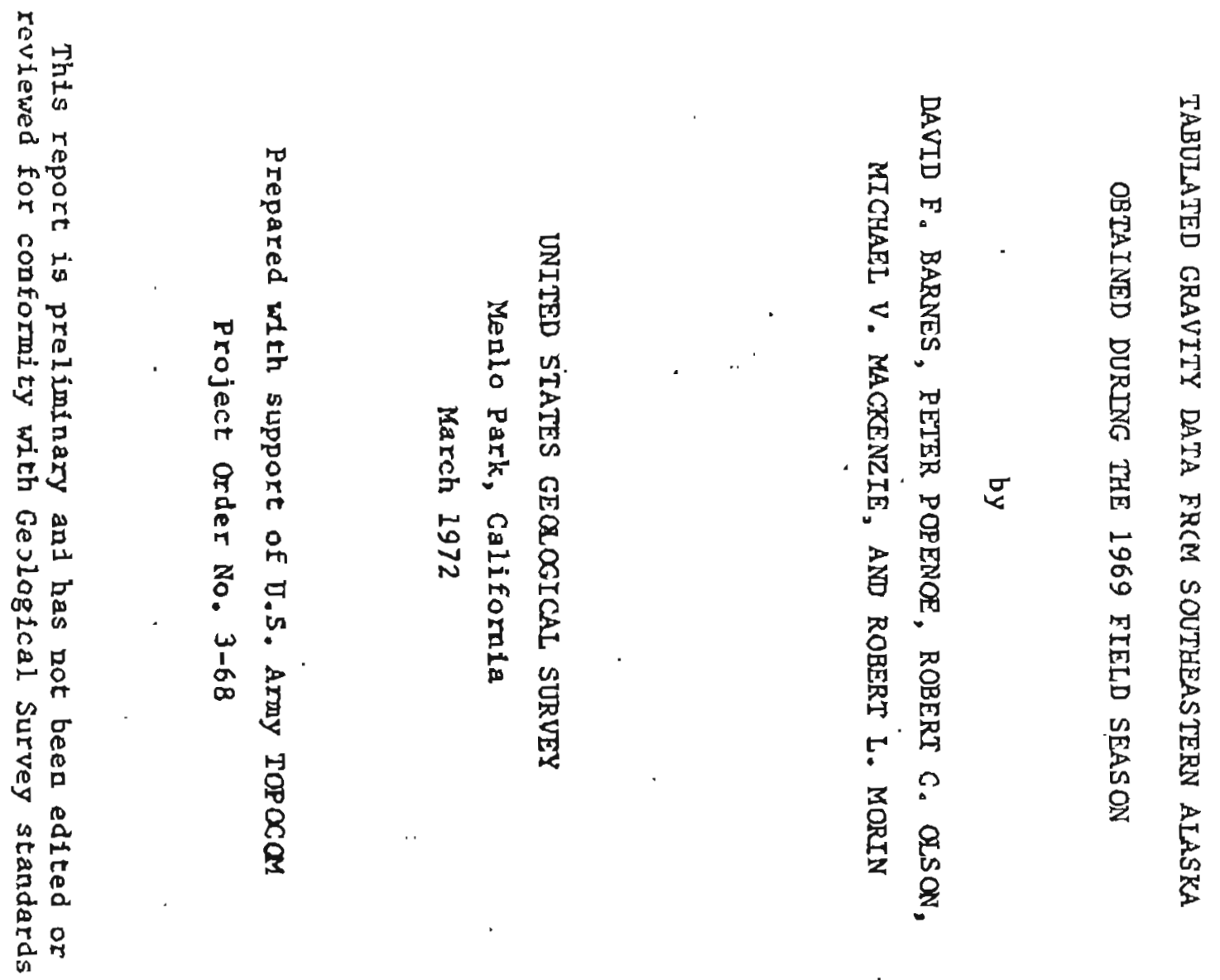


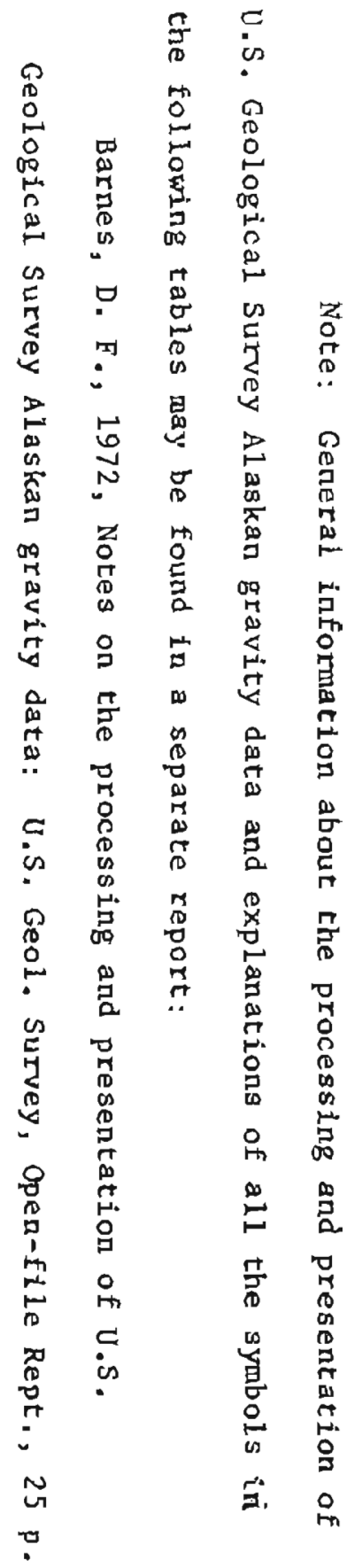




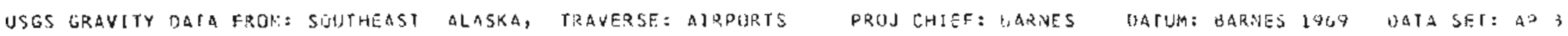

STAT. VOS.

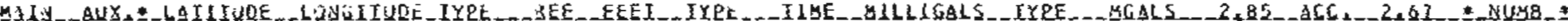

\begin{tabular}{|c|c|c|c|c|c|c|c|c|c|c|c|c|c|c|c|c|c|c|c|c|}
\hline $1 \mathrm{KY}$ & DESE & $\# 51$ & 2.99 & 135 & 20.25 & A & 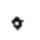 & 0 & 23 & [ & $\neq$ & 0850 & 981707.12 & $\mathrm{~B}$ & $*$ & 22.7 & 21.9 & 4 & 22.0 & - $57 K$ \\
\hline$T K B$ & BASE & $* 57$ & 3.12 & $1 \pm 5$ & 21.75 & $a$ & * & 0 & 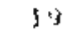 & $\xi$ & $\$$ & 0924 & 981708.28 & $\mathbf{A}$ & - & 23.5 & 22.8 & 4 & 22.9 & - SIKG \\
\hline & BASE & $\begin{array}{l}-56 \\
\end{array}$ & 49.40 & 132 & 56.11 & 3 & * & 0 & 100 & $\mathrm{~F}$ & * & 1024 & 901652.40 & $\theta$ & * & -4.4 & -6.1 & 7 & $-7 * 8$ & * PETS \\
\hline PETH & QASE & * 56 & 48.82 & $1 \pm 2$ & 57.30 & $A$ & * & 0 & 16 & $\mathrm{C}$ & * & 1059 & 981659.01 & $A$ & * & -6.3 & -6.9 & 4 & -6.8 & * PETH \\
\hline TY & Tol1 & * 56 & 48.83 & 132 & 57.33 & a. & 4 & 0 & 16 & A & * & 1102 & 991659.90 & A & $*$ & -6.4 & -7.0 & 4 & -7.0 & * PETI \\
\hline ETH & BASE & *56 & 48.87 & 132 & 57.30 & $A$ & \# & 0 & 16 & $c$ & $*$ & 1440 & 981659.01 & A & 4 & -6.3 & -6.9 & 4 & $-6+8$ & * PETH \\
\hline$T N$ & BASE & $* 58$ & 48.40 & 132 & 50.12 & $\beta$ & $*$ & 0 & 100 & $\mathrm{E}$ & \# & 1459 & 781052.40 & B & * & -4.4 & $-R+1$ & 4 & -7.8 & * $P \subseteq T$ \\
\hline RiN & GASE & $* 56$ & 29.17 & 152 & 22.64 & 4 & $\Rightarrow$ & 0 & 6,3 & e & \# & 1516 & 981615.45 & a & \# & -10.4 & -20.6 & 4 & -20.5 & $\# W M T$ \\
\hline NET & BASE & $\neq 55$ & 2.24 & 131 & 34.60 & A & * & 0 & 219 & $E$ & * & 1628 & 981527.52 & A & $*$ & 20.9 & 16.6 & 4 & 16.2 & * ANE \\
\hline VEH & AASE & +55 & 2.29 & 131 & 34.60 & $\mathbf{A}$ & * & 0 & 119 & $E$ & * & 2840 & 981.527 .63 & a & * & 21.0 & 16.7 & 4 & 16.9 & - ANE \\
\hline IEF & FUTD & - 55 & 2.30 & 131 & 34.61 & $A$ & a & 0 & $11 \%$ & $E$ & - & 1652 & 986527.41 & $c$ & $*$ & $20 . a$ & 16.4 & 4 & 16.7 & - AXEF \\
\hline AQE T & BASE & $* 55$ & 2.29 & 131 & 34.60 & A & * & 0 & 119 & $E$ & - & 1656 & 981527.52 & A & * & 20.9 & 16.6 & 4 & 16.8 & \# ARKE \\
\hline $\operatorname{tef}$ & BASE & 755 & 20.73 & 131 & 39.45 & $\Delta$ & * & 0 & 16 & C & $*$ & 1751 & 781543.64 & $A$ & - & 1.3 & 0.7 & 4 & 0.8 & * KET \\
\hline
\end{tabular}

QAIB_SLHABYY

VUMAER OF STATHNS: 13

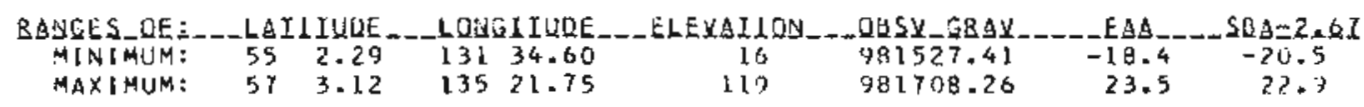

USGS GRAVITY DATA FROK: SOUTHEAST ALASKA, TRAYERSE: LULU-NOYES gROJ CHIEF: BARAS DATUM: BARNES I9TI DATA SET: GPOS

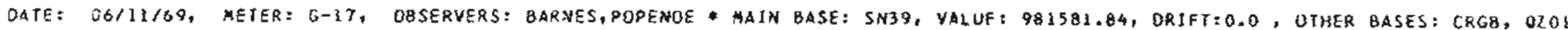

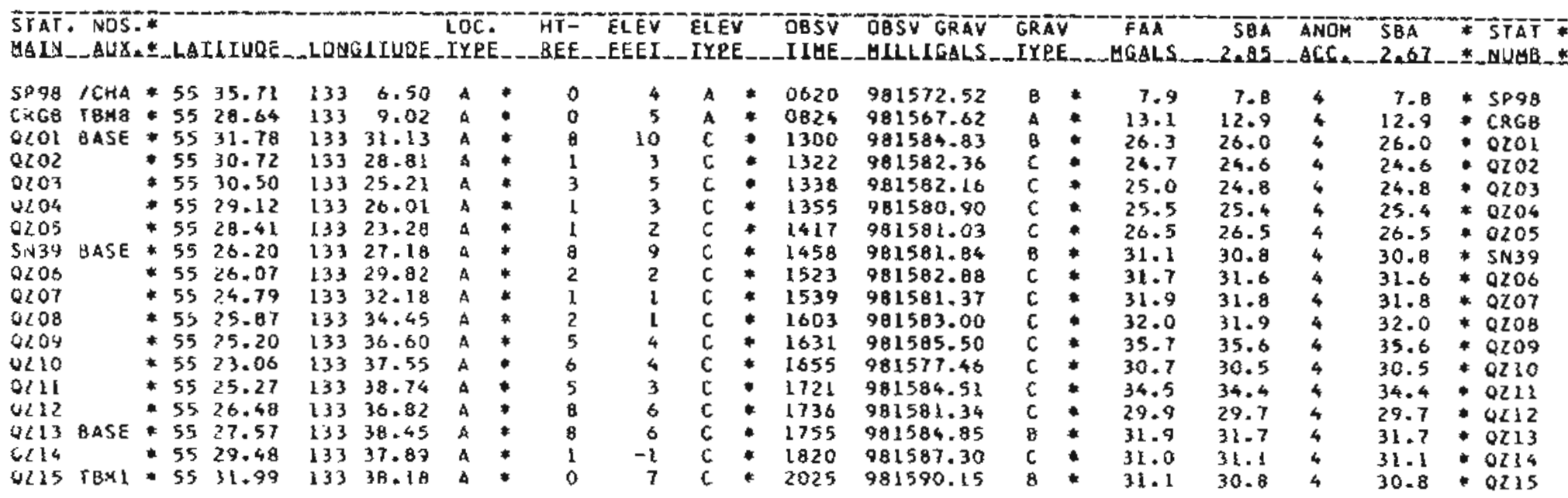




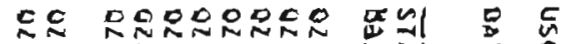

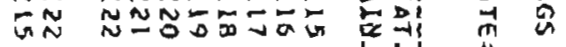

a

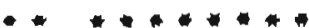

un unumunu

(1)

๘"

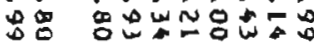

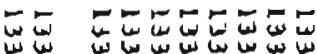

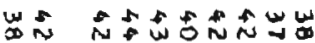

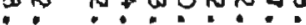

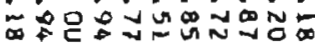

等

窟

D国D吅D

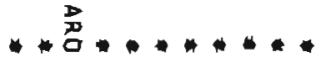

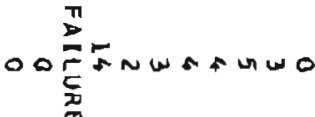

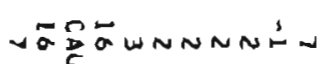

c

pronanomant

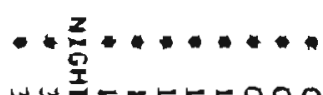

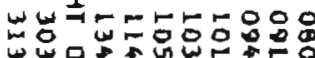

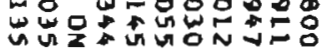

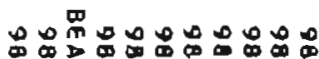

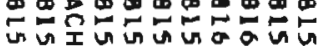

品

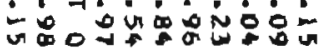

的是

!

品品

ank

鲴

的最

w出

:

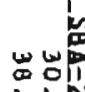

is

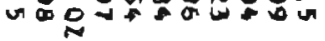
$00 \stackrel{N}{N} 00000000$

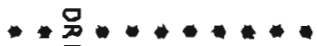

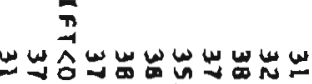

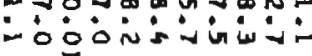

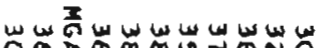

$\because \geqslant 0.0 \%$

$\infty$ in - wa:Na

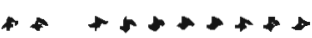

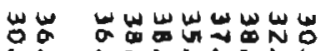

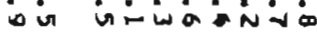

- H. H.

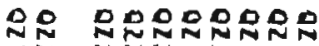

UN NNNOTENTE

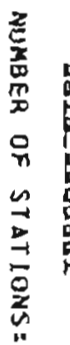

5

焉

뎐

m?

$x$

mi

$m \frac{m}{m}$

$\rightarrow<$

$4 m$

空

造密

to $z-$

$5 \approx$

2:

to

然

L

策

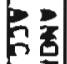

궁훙

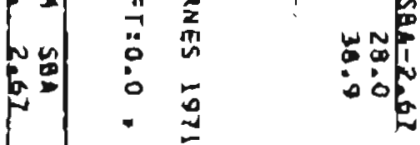

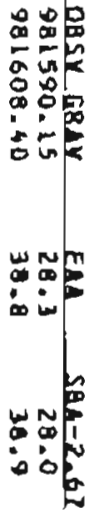

:

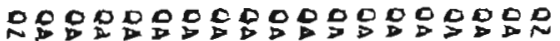

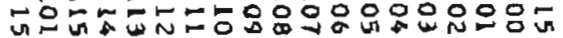

至 $\overrightarrow{\underline{X}}$

资

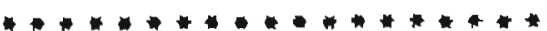

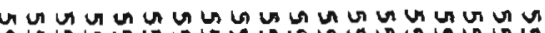

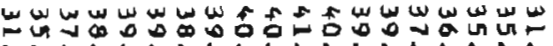

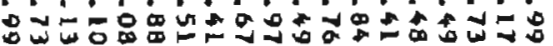

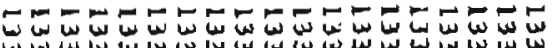

出

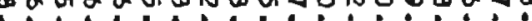

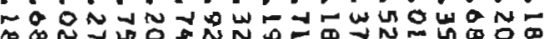

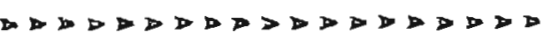

$\ldots \ldots \ldots \ldots \ldots \ldots \ldots$

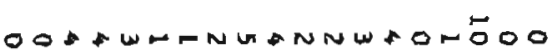

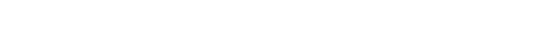

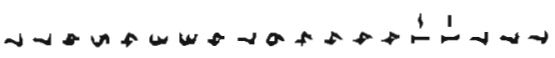

monannammannannan

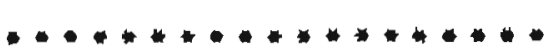

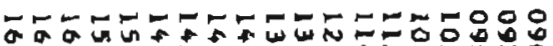

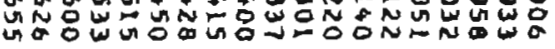

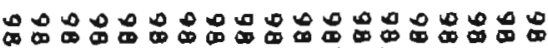

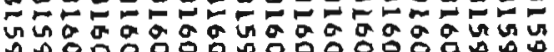

Ond

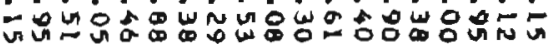

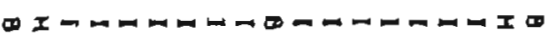

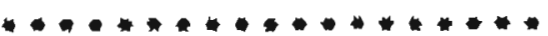

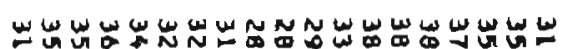

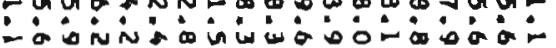

س

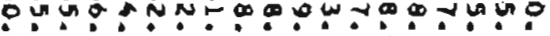

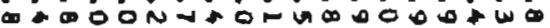

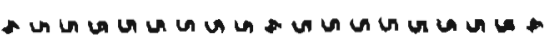

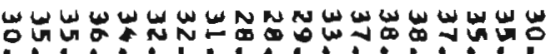

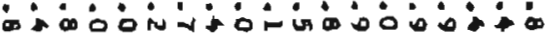

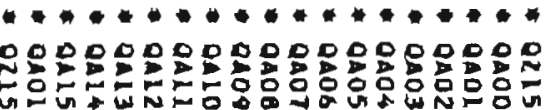




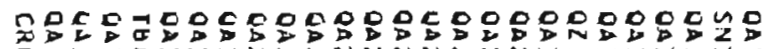

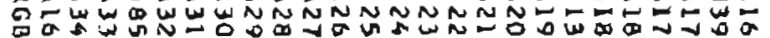
要总焉焉

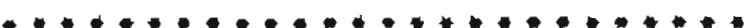

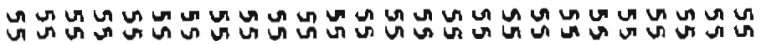

NNWw出 ON- N N

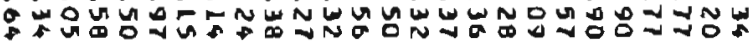

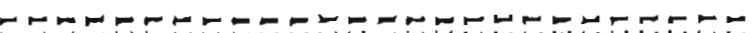
س

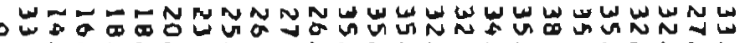

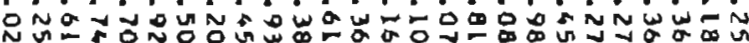

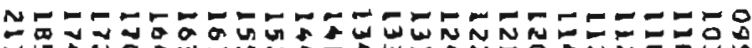

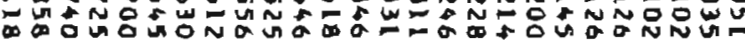

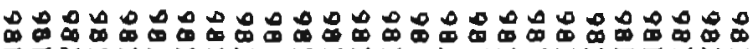
ง

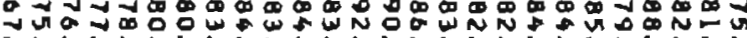
- i

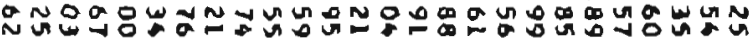

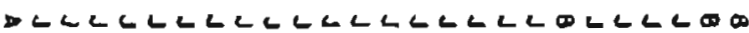

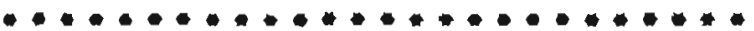

テ ็っ

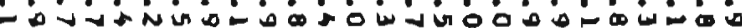

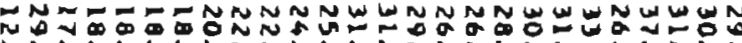

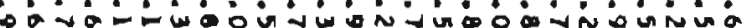

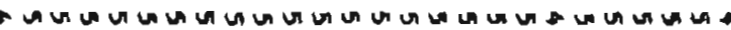

ニ ó

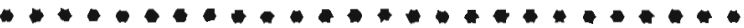

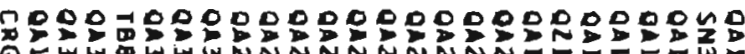

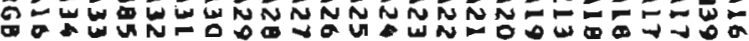




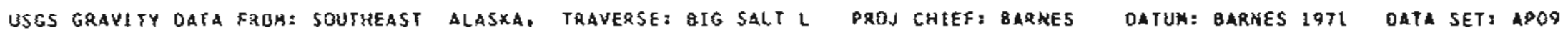
DATE: 06/15/69, METEA: W226, OQSERVERS: POPENOE

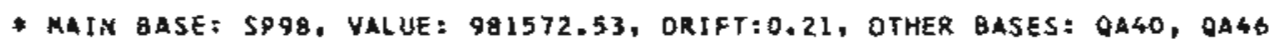

\begin{tabular}{|c|c|c|c|c|c|c|c|c|c|c|c|c|c|c|c|c|c|c|}
\hline DAIS- & 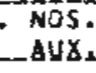 & & & 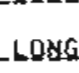 & & & & EF & $\mathrm{E}$ & & $\begin{array}{l}\text { OBSV } \\
\text { ILHE. }\end{array}$ & $\begin{array}{l}\text { OBSY GRAY } \\
\text { BLWLIGALS }\end{array}$ & & $\begin{array}{l}\text { FAA } \\
\text { HGALS. }\end{array}$ & $\begin{array}{r}564 \\
2.85 \\
\end{array}$ & ANOH & $A$ & $\begin{array}{l}\text { STAT } \\
+\quad \text { MUHA }\end{array}$ \\
\hline $\begin{array}{l}\text { CRGB } \\
\text { SP9B } \\
\text { QA35 } \\
\text { QA36 } \\
\text { QA37 } \\
\text { QA38 } \\
\text { QA39 } \\
\text { QA40 } \\
\text { QA41 } \\
\text { QA42 } \\
\text { QA43 } \\
\text { QA44 } \\
\text { QA45 } \\
\text { QA46 } \\
\text { SP9B } \\
\text { QA47 } \\
\text { QA49 } \\
\text { QA49 } \\
\text { QA50 } \\
\text { QA51 } \\
\text { QA52 } \\
\text { SP98 }\end{array}$ & $\begin{array}{l}\text { FOTO } \\
\text { BASE }\end{array}$ & $\begin{array}{l}\quad 55 \\
* 55 \\
* 55 \\
* 55 \\
* 55 \\
* 55 \\
* 55 \\
* 55 \\
* 55 \\
* 55 \\
* 55 \\
* 55 \\
* 55 \\
* 55 \\
* 55 \\
* 55 \\
* 55 \\
* 55 \\
* 55 \\
* 55 \\
* 55 \\
* 55\end{array}$ & $\begin{array}{l}28.64 \\
35.71 \\
36.62 \\
35.95 \\
34.99 \\
34.78 \\
34.14 \\
34.07 \\
33.02 \\
34.61 \\
35.42 \\
35.71 \\
36.53 \\
36.97 \\
35.71 \\
37.38 \\
37.04 \\
37.23 \\
37.06 \\
37.29 \\
35.93 \\
35.71\end{array}$ & $\begin{array}{l}133 \\
133 \\
133 \\
133 \\
133 \\
133 \\
133 \\
133 \\
133 \\
133 \\
133 \\
133 \\
133 \\
133 \\
133 \\
133 \\
132 \\
132 \\
133 \\
133 \\
133 \\
133\end{array}$ & $\begin{array}{r}9.02 \\
6.50 \\
7.47 \\
9.29 \\
10.87 \\
12.74 \\
14.02 \\
11.39 \\
13.65 \\
16.21 \\
18.04 \\
19.77 \\
21.31 \\
24.28 \\
6.50 \\
0.24 \\
59.05 \\
56.11 \\
2.03 \\
4.19 \\
2.35 \\
6.50\end{array}$ & $\begin{array}{l}A \\
A \\
A \\
A \\
A \\
A \\
A \\
A \\
A \\
A \\
A \\
A \\
A \\
A \\
A \\
A \\
A \\
A \\
A \\
A \\
A \\
A\end{array}$ & $\begin{array}{l}* \\
* \\
* \\
* \\
* \\
* \\
* \\
* \\
* \\
* \\
* \\
* \\
* \\
* \\
* \\
* \\
* \\
* \\
*\end{array}$ & $\begin{array}{r}0 \\
0 \\
1 \\
1 \\
6 \\
1 \\
1 \\
10 \\
1 \\
2 \\
5 \\
1 \\
1 \\
3 \\
0 \\
0 \\
0 \\
0 \\
1 \\
0 \\
0 \\
3\end{array}$ & $\begin{array}{r}5 \\
4 \\
-5 \\
-5 \\
1 \\
-3 \\
-3 \\
7 \\
-1 \\
1 \\
5 \\
1 \\
2 \\
5 \\
4 \\
3 \\
3 \\
3 \\
3 \\
2 \\
2 \\
4\end{array}$ & $\begin{array}{l}A \\
A \\
C \\
C \\
C \\
C \\
C \\
C \\
C \\
C \\
C \\
C \\
C \\
C \\
A \\
C \\
C \\
C \\
C \\
C \\
C \\
C\end{array}$ & 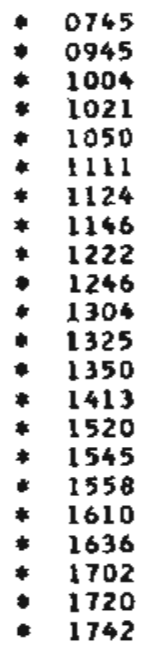 & $\begin{array}{l}981567.62 \\
981572.53 \\
981573.64 \\
981573.84 \\
981574.81 \\
981577.69 \\
981578.04 \\
981575.00 \\
981576.27 \\
981579.55 \\
981581.17 \\
981581.77 \\
981583.02 \\
981586.52 \\
981572.53 \\
981574.43 \\
981575.26 \\
981572.94 \\
981575.15 \\
981569.40 \\
981573.22 \\
981572.53\end{array}$ & $\begin{array}{l}1 \\
1 \\
I \\
I \\
I \\
I \\
I \\
B \\
I \\
1 \\
I \\
I \\
I \\
I \\
1 \\
1 \\
1 \\
I \\
1 \\
\text { I } \\
1 \\
\text { I }\end{array}$ & $\begin{array}{r}13.0 \\
15.0 \\
16.2 \\
17.1 \\
16.9 \\
17.1 \\
20.3 \\
0.0 \\
7.4 \\
7.8 \\
6.1 \\
8.6 \\
2.4 \\
8.1 \\
8.0\end{array}$ & $\begin{array}{l}13.9 \\
15.1 \\
12.8 \\
15.0 \\
16.2 \\
16.9 \\
16.9 \\
17.0 \\
20.1 \\
7.8 \\
7.3 \\
7.5 \\
6.0 \\
8.5 \\
2.3 \\
6.1 \\
7.8\end{array}$ & $\begin{array}{l}4 \\
4 \\
5 \\
5 \\
5 \\
5 \\
5 \\
4 \\
5 \\
5 \\
5 \\
5 \\
5 \\
5 \\
4 \\
5 \\
5 \\
5 \\
4 \\
4 \\
4 \\
5\end{array}$ & $\begin{array}{r}12.9 \\
7.9 \\
7.1 \\
1.3 \\
12.1 \\
13.9 \\
15.1 \\
12.8 \\
15.0 \\
16.2 \\
16.9 \\
16.9 \\
17.0 \\
20.1 \\
7.8 \\
7.3 \\
7.5 \\
6.0 \\
8.5 \\
2.4 \\
0.1 \\
7.8\end{array}$ & 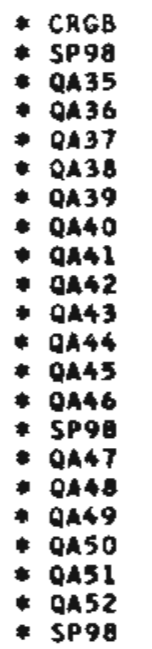 \\
\hline
\end{tabular}

QAIA_SUHUABY

HUMBER OF STATIONS: 22

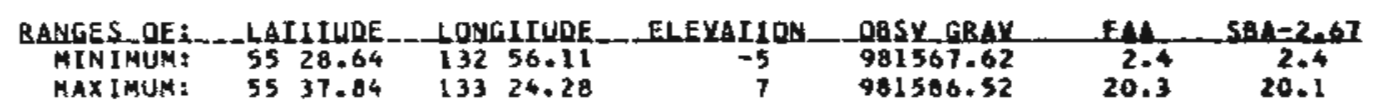


USGS GRAYITY OATA FROM: SUUTHEAST ALASKA, TRAVERSE: W BAKER IS PAOJ CHIEF: BARNES DATUMI BARNES 197L DATA SET: APLO

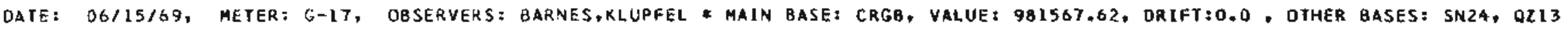

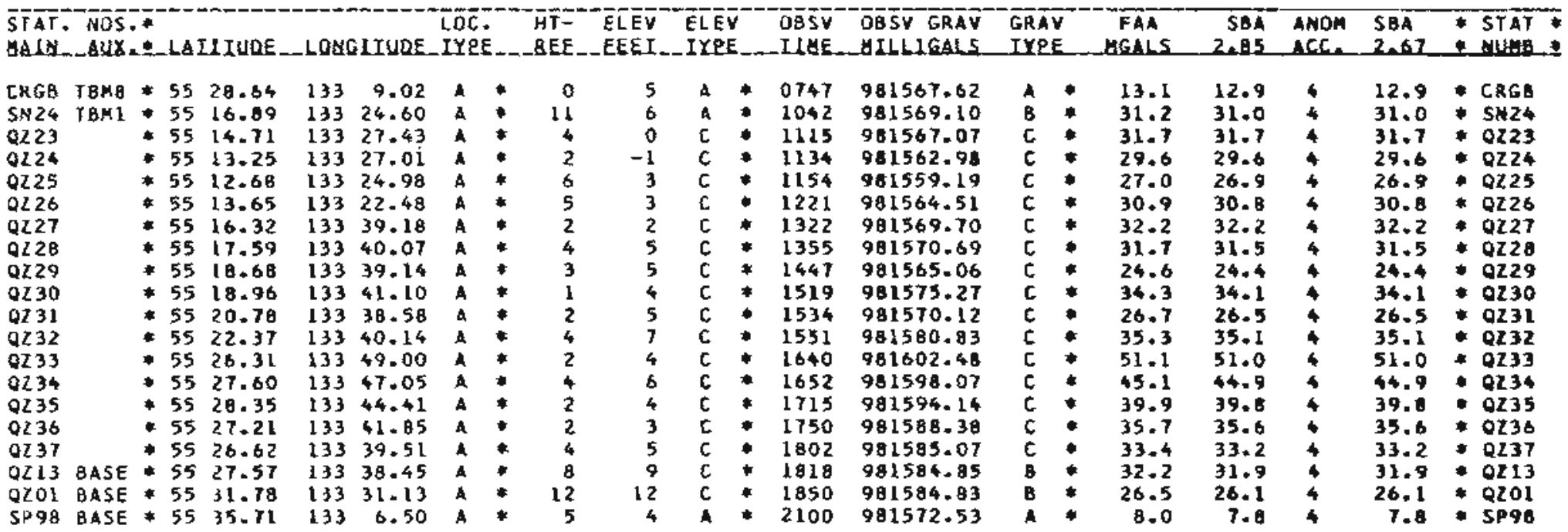

DAIA_SUHABRY

MUHREH DF STAIIDNS: 20

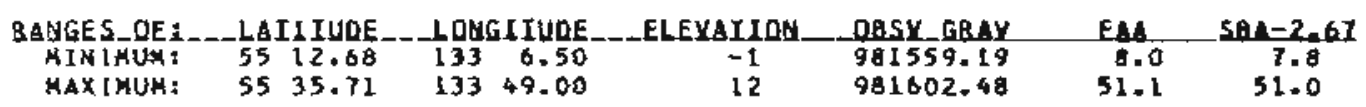


USGS GRAVIT DATA FROM: STUTHEAST ALASKA, FHAVERSE TU CYRUS C PROS CIIEF: BARNES DATUM; BARNES I9TI DATA SET: APII

\begin{tabular}{|c|c|c|c|c|c|c|c|}
\hline $\operatorname{SPQ\theta }$ & BASE & - 55 & 35.71 & 133 & 6.50 & A & * \\
\hline QA40 & I & $* 55$ & 36.07 & 133 & 11.39 & A & \\
\hline TB85 & Твн2 & $\neq 55$ & 32.50 & 133 & 18.70 & A & * \\
\hline $9<38$ & TBM1 & +55 & 32.50 & 133 & 18.70 & A & * \\
\hline Q446 & FOTO & 455 & 16.97 & 133 & 24.28 & A. & * \\
\hline 9239 & FOTO & $* 55$ & 38.13 & 133 & 24.20 & A & * \\
\hline$Q \angle 40$ & & $* 55$ & 39.95 & 133 & 23.51 & A & * \\
\hline $0<41$ & & $* 55$ & 41.16 & 133 & 21.40 & 4 & • \\
\hline$Q 242$ & & $\neq 55$ & 41.90 & 133 & 23.84 & A & - \\
\hline 0400 & J日H I & * 55 & 40.97 & 133 & 35.14 & $\mathbf{A}$ & $\bullet$ \\
\hline $0<43$ & & +55 & 43.82 & 133 & 29.17 & A & * \\
\hline$Q 244$ & & $\div 55$ & 45.36 & 133 & 30.67 & A & $*$ \\
\hline $0 \angle 45$ & & $* 55$ & 46.42 & 133 & 33.03 & A & * \\
\hline 0246 & & $\div 55$ & 46.99 & 133 & 30.49 & A & • \\
\hline 0247 & & $* 55$ & 45.13 & 133 & 27.35 & A & * \\
\hline 0248 & & 755 & 44.48 & $1+3$ & 24.91 & $A$ & $*$ \\
\hline$Q<49$ & & - 55 & 43.40 & 133 & 24.85 & $A$ & * \\
\hline $0<50$ & $/ \varsigma^{9} \mid$ & $\begin{array}{l}+55 \\
\end{array}$ & 44.20 & 133 & 22.99 & A & * \\
\hline $0<51$ & & & 43.01 & 133 & $21=18$ & A & - \\
\hline 0252 & & * 55 & 42.11 & 133 & 19.25 & A & $*$ \\
\hline $0 \angle 53$ & & $\div 55$ & $44-31$ & 133 & 19.95 & A & $*$ \\
\hline $0 \angle 54$ & & $* 55$ & 45.61 & 113 & 22.12 & A & * \\
\hline 0255 & / SUR & * 55 & 46.03 & 133 & 19.80 & A & $*$ \\
\hline 0456 & & & 44.52 & 133 & 17.04 & A & $*$ \\
\hline 4257 & & +55 & $45-50$ & 133 & 14.97 & A & * \\
\hline OL 59 & & $* 55$ & $4 b=57$ & 133 & 13.70 & A & $*$ \\
\hline $4 / 59$ & & * 55 & 47.75 & 133 & 11.09 & A & * \\
\hline$a t 60$ & TBH1 & * 55 & 40.67 & 133 & 10.61 & 4 & * \\
\hline 0261 & & $* 55$ & 47.07 & 133 & 14.63 & A & * \\
\hline $4 Z 62$ & BASE & $* 55$ & 46.19 & 133 & 17.03 & A & * \\
\hline 0263 & & 55 & 47.48 & 133 & 17.45 & A & * \\
\hline 4264 & & +52 & 48.42 & 133 & 19.71 & A & - \\
\hline 9265 & BASE & 455 & 55.21 & 133 & 24.63 & A & 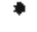 \\
\hline
\end{tabular}

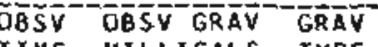

$\bar{F} \bar{A} \bar{A}$

SBA ANDA SBA $\overline{S T A T}$

ELEV ELE
EEEI_IVE

ISE BLLIGALS

MGALS 20 S ACC. $2 . B I$ HUMB

QAIA_SUEMABI

NUMBER DF STATIONS: 33

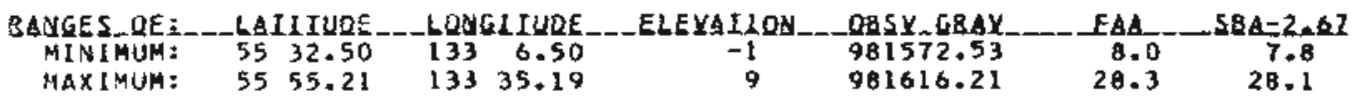

\begin{tabular}{|c|c|c|c|c|}
\hline 6.0 & $\begin{array}{l}7.8 \\
12.7 \\
18.1 \\
18.2 \\
20.1 \\
21.0 \\
21.1 \\
21.7 \\
24.2 \\
28.1 \\
24.6 \\
23.5 \\
26.2 \\
28.1 \\
24.8 \\
20.9 \\
26.3 \\
26.5 \\
24.5 \\
21.1 \\
24.2 \\
24.8 \\
24.9 \\
21.6 \\
25.6 \\
28.0 \\
24.6 \\
23.6 \\
26.6 \\
23.4 \\
25.2 \\
24.4 \\
24.3\end{array}$ & $\begin{array}{l}4 \\
4 \\
4 \\
4 \\
4 \\
4 \\
4 \\
4 \\
4 \\
4 \\
4 \\
4 \\
4 \\
4 \\
4 \\
4 \\
4 \\
4 \\
4 \\
4 \\
4 \\
4 \\
4 \\
4 \\
4 \\
4 \\
4 \\
4 \\
4 \\
4 \\
4 \\
4 \\
4\end{array}$ & $\begin{array}{l}7.8 \\
12.8 \\
18.2 \\
18.2 \\
20.1 \\
21.0 \\
21.1 \\
21.7 \\
24.2 \\
28.1 \\
24.6 \\
23.5 \\
26.2 \\
28.1 \\
24.8 \\
20.9 \\
26.3 \\
26.5 \\
24.5 \\
21.1 \\
24.2 \\
24.8 \\
25.0 \\
21.7 \\
25.6 \\
28.0 \\
24.6 \\
23.7 \\
26.6 \\
23.4 \\
25.2 \\
24.4 \\
24.3\end{array}$ & 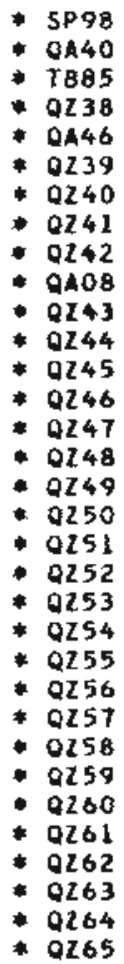 \\
\hline
\end{tabular}

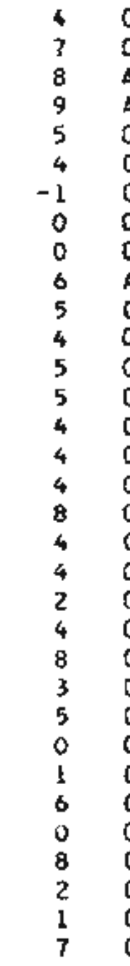

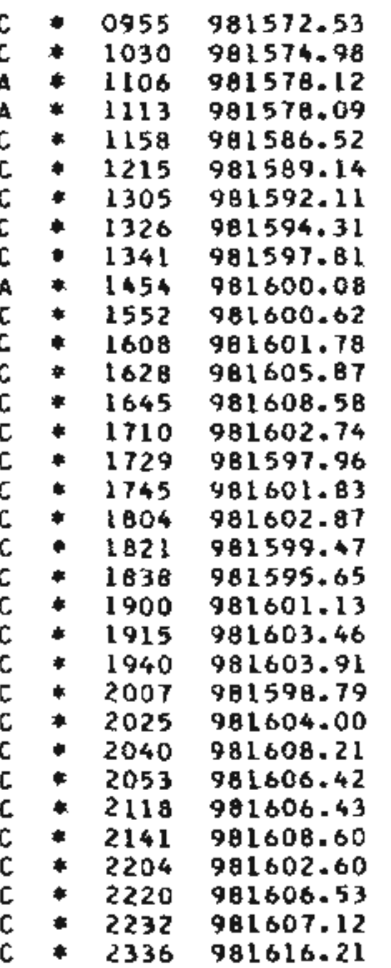

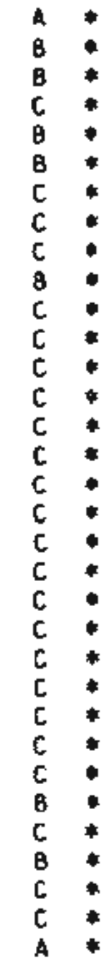

24.6

$\begin{array}{rr}6.0 & 7.8 \\ 13.0 & 12.7 \\ 18.4 & 18.1 \\ 18.5 & 18.2 \\ 20.3 & 20.1 \\ 21.2 & 21.0 \\ 21.1 & 21.1 \\ 21.7 & 21.7 \\ 24.2 & 24.2 \\ 28.3 & 28.1 \\ 24.7 & 24.6 \\ 23.7 & 23.5 \\ 26.4 & 26.2 \\ 28.3 & 28.1 \\ 24.9 & 24.8 \\ 21.1 & 20.9 \\ 26.5 & 26.3 \\ 26.7 & 26.5 \\ 24.6 & 24.5 \\ 21.3 & 21.1 \\ 24.3 & 24.2 \\ 25.0 & 24.8 \\ 25.2 & 24.9 \\ 21.9 & 21.6 \\ 25.8 & 25.6 \\ 20.0 & 28.0 \\ 24.7 & 24.6 \\ 23.9 & 23.6 \\ 26.6 & 26.6 \\ 23.7 & 23.4 \\ 25.3 & 25.2 \\ 24.4 & 24.4 \\ 24.6 & 24.3 \\ & \end{array}$






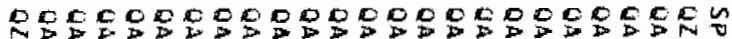

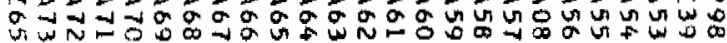
焉变

票䆑

$\underset{\mathbf{n}}{\mathbf{0}}$

勇密

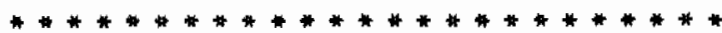

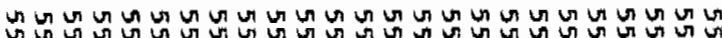

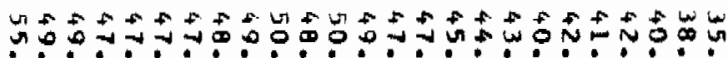

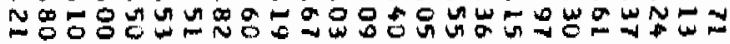

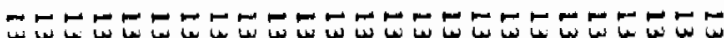

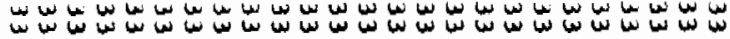

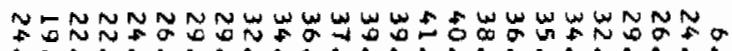

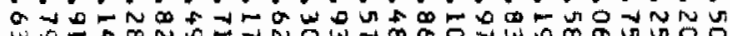

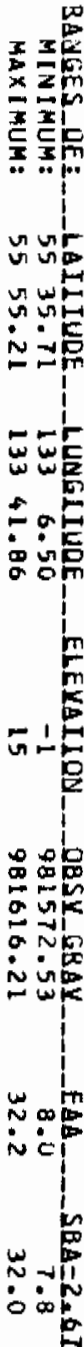

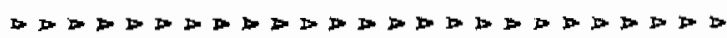

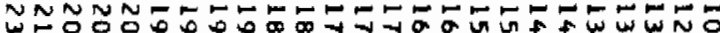

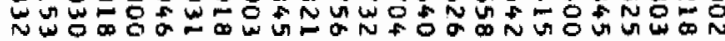

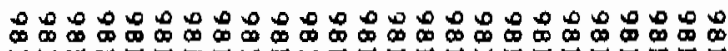

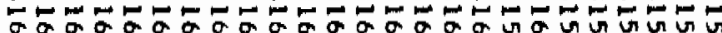

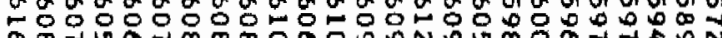

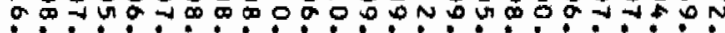

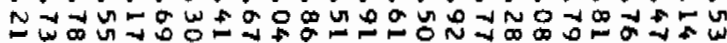

\$

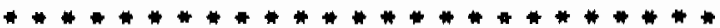

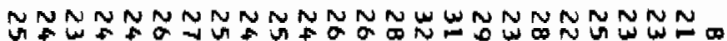

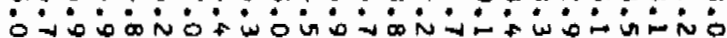

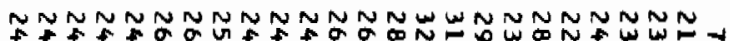

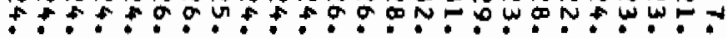
$0+0$ - 1 N 0 w ง

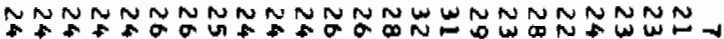

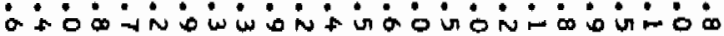

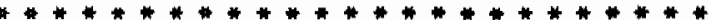

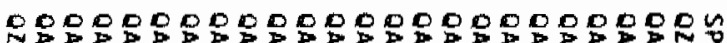

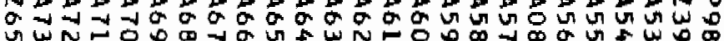


USGS GRAVITY JATA FROM: SUUTHEAST ALASKA, TRAYER5E: TDKEEN BAY PRUJ CHIEF: GAP.NES DATUH: WARNES L97I DATA SET: API3

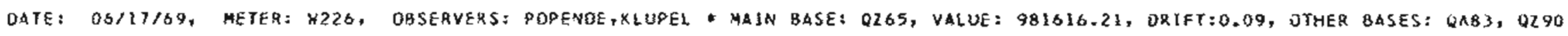

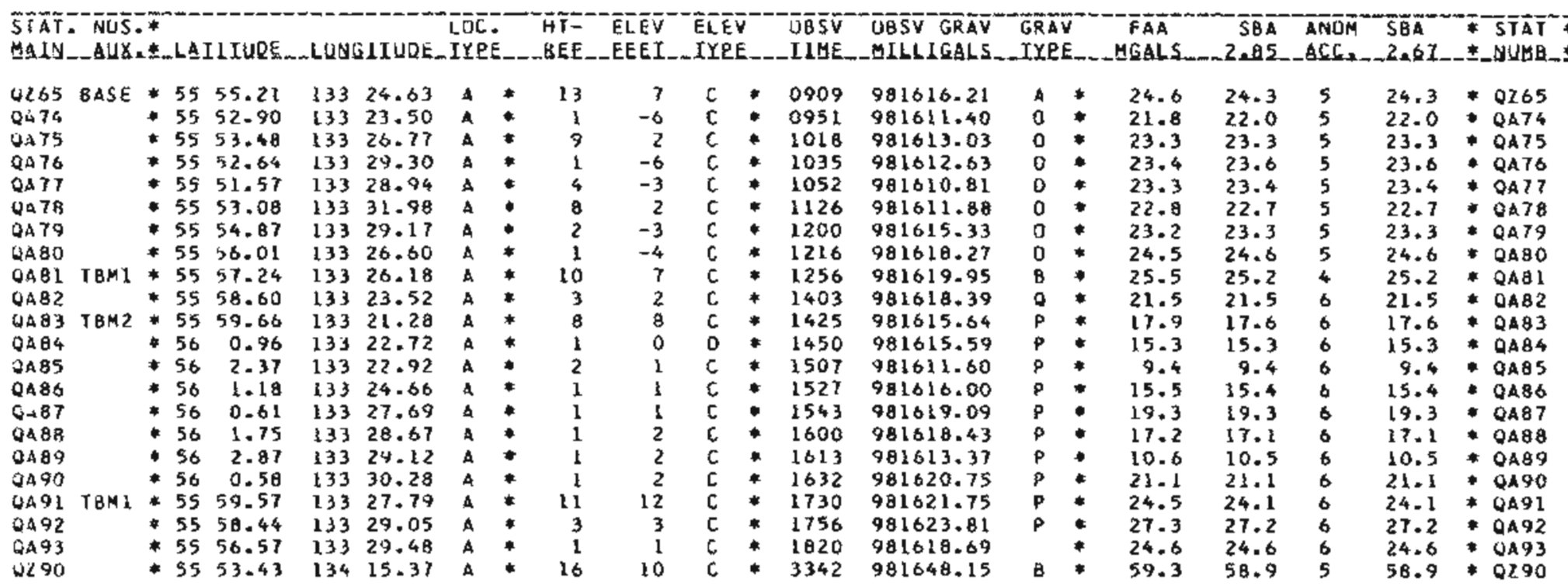

NEEO TO ASSIST A OISABLED SKIFF PREYENTED CLOSURE GF TRAUERES ON G/1T/O9

FOLLOWING HORMING DRIFT WAS +0.4 MGALS WHICH DECREASE TO 0.0 AT OHYIS END

DAIA_SUHBABY

NUMEER OF SIATIIJNS: 22

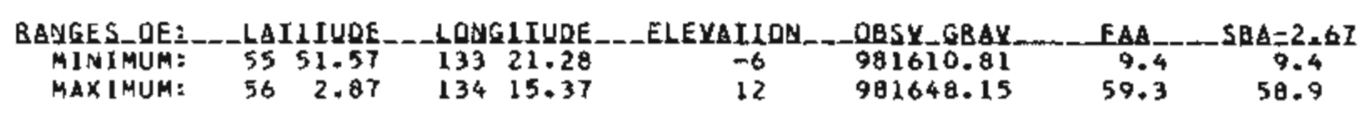




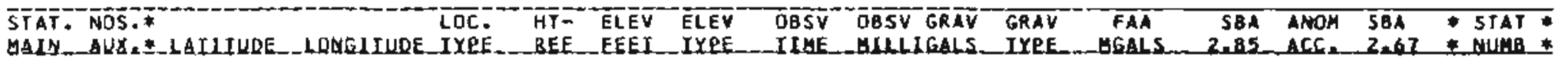

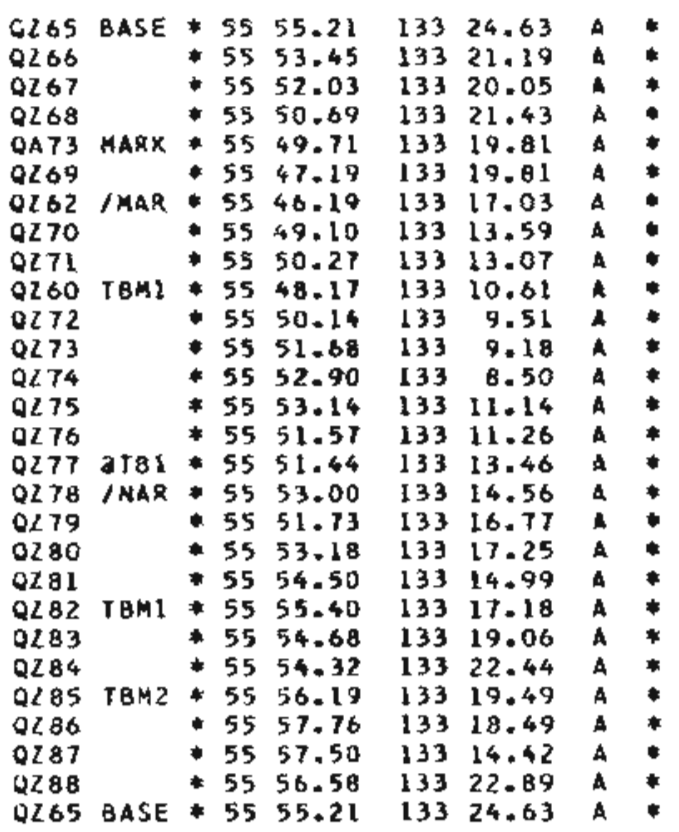

14
3
5
2
12
2
12
3
2
12
2
3
5
1
3
4
4
4
2
3
5
2
2
5
3
3
2
0

$\begin{array}{rr}7 & A \\ -4 & C \\ -2 & C \\ -5 & C \\ 5 & C \\ -4 & 6 \\ 6 & C \\ -3 & 6 \\ -3 & C \\ 7 & 4 \\ -2 & 6 \\ -1 & 6 \\ 2 & C \\ -2 & C \\ 1 & C \\ 3 & 4 \\ 4 & C \\ 5 & C \\ 3 & C \\ 5 & 6 \\ 7 & 4 \\ 5 & C \\ 5 & C \\ 8 & A \\ 6 & C \\ 6 & 6 \\ 4 & 0 \\ 7 & C \\ & \end{array}$

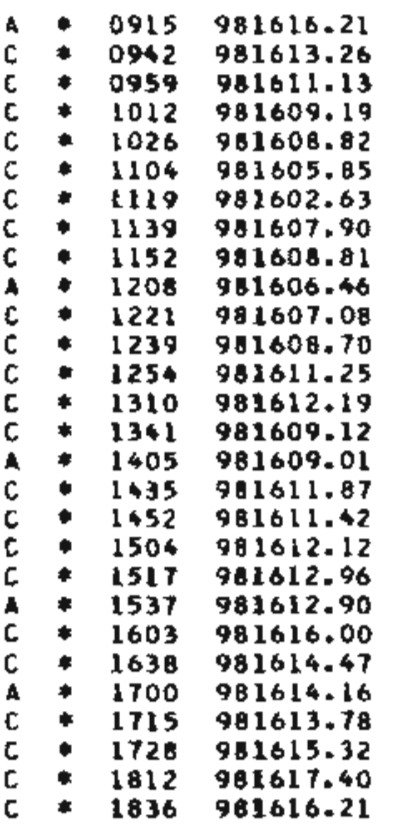

D
0
0
0
0
0
0
0

$\begin{array}{lll}24.6 & 24.3 & 4 \\ 23.1 & 23.2 & 4 \\ 23.1 & 23.2 & 4 \\ 22.8 & 22.9 & 4 \\ 24.7 & 24.5 & 4 \\ 24.4 & 24.6 & 4 \\ 23.5 & 23.3 & 4 \\ 23.9 & 24.0 & 4 \\ 23.2 & 23.3 & 4 \\ 24.7 & 24.4 & 4 \\ 21.7 & 21.8 & 4 \\ 21.3 & 21.3 & 4 \\ 22.4 & 22.3 & 4 \\ 22.6 & 22.7 & 4 \\ 22.9 & 22.0 & 4 \\ 22.3 & 22.2 & 4 \\ 23.1 & 22.9 & 4 \\ 24.5 & 24.3 & 4 \\ 23.0 & 22.8 & 4 \\ 22.1 & 21.9 & 4 \\ 21.0 & 20.7 & 4 \\ 24.9 & 24.7 & 4 \\ 23.9 & 23.7 & 4 \\ 21.3 & 21.0 & 4 \\ 18.5 & 18.3 & 4 \\ 20.4 & 20.2 & 4 \\ 23.6 & 23.4 & 4 \\ 24.6 & 24.3 & 4\end{array}$

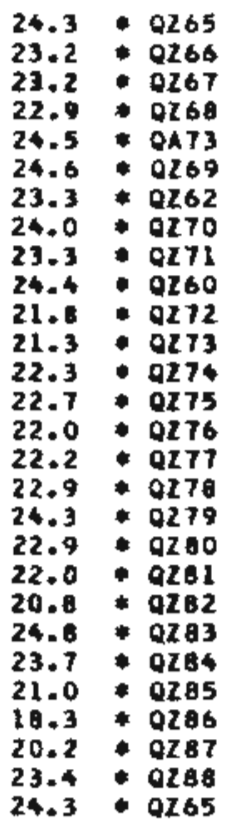

QAIA_SUUUAGY

NUABER OF STATIUAS: 28

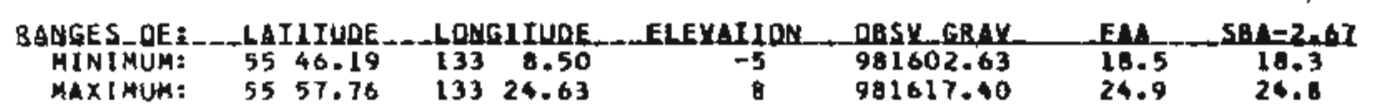




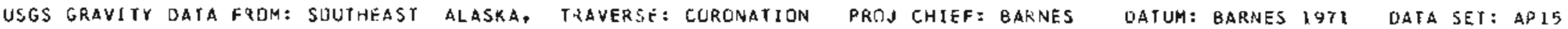

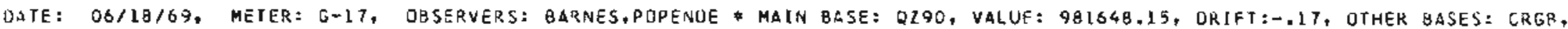

\begin{tabular}{|c|c|c|c|c|c|c|c|c|c|c|c|c|c|c|c|c|c|c|c|c|}
\hline $\begin{array}{l}\text { SIA } \\
\text { SAI }\end{array}$ & 40 & 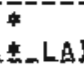 & (1) & $\mathrm{N}$ & & & & $1-$ & & & & 08 & $\begin{array}{l}0 \bar{B} \bar{G} \\
\text { ULLLGA }\end{array}$ & & & $\mathrm{FA}$ & $\begin{array}{r}\mathrm{SBA} \\
2.85\end{array}$ & & 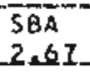 & 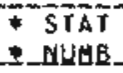 \\
\hline $\begin{array}{l}4290 \\
0291 \\
4292 \\
0293 \\
0290 \\
0368\end{array}$ & $\begin{array}{l}\text { BASE } \\
\text { TBM2 }\end{array}$ & $\begin{array}{l}* 55 \\
* \quad 55 \\
* 55 \\
* 55 \\
* 55 \\
* \quad 55\end{array}$ & $\begin{array}{l}53.43 \\
54.71 \\
55.70 \\
52.60 \\
53.43 \\
28.64\end{array}$ & $\begin{array}{l}134 \\
134 \\
134 \\
134 \\
134 \\
133\end{array}$ & $\begin{array}{r}15.37 \\
18.53 \\
20.01 \\
16.99 \\
15.37 \\
9.02\end{array}$ & $\begin{array}{l}A \\
A \\
A \\
A \\
A \\
A\end{array}$ & $\begin{array}{l}* \\
* \\
* \\
*\end{array}$ & $\begin{array}{r}16 \\
15 \\
5 \\
3 \\
0 \\
0\end{array}$ & $\begin{array}{r}10 \\
8 \\
-2 \\
2 \\
10 \\
5\end{array}$ & $\begin{array}{l}c \\
c \\
c \\
c \\
c \\
A\end{array}$ & $*$ & $\begin{array}{l}0924 \\
1028 \\
1046 \\
1425 \\
1528 \\
2225\end{array}$ & $\begin{array}{l}981648.15 \\
981651.66 \\
981653.93 \\
961646.28 \\
981648.15 \\
981567.62\end{array}$ & $\begin{array}{l}6 \\
C \\
C \\
C \\
B \\
A\end{array}$ & $\begin{array}{l}* \\
* \\
* \\
*\end{array}$ & $\begin{array}{l}59.3 \\
60.8 \\
60.8 \\
57.8 \\
59.3 \\
13.1\end{array}$ & $\begin{array}{l}58.9 \\
60.5 \\
60.8 \\
57.8 \\
58.9 \\
12.9\end{array}$ & $\begin{array}{l}4 \\
4 \\
4 \\
4 \\
4 \\
4\end{array}$ & $\begin{array}{l}58.9 \\
60.5 \\
60.6 \\
57.8 \\
58.9 \\
12.9\end{array}$ & $\begin{array}{r}\text { +290 } \\
+\quad 291 \\
+\quad 292 \\
+\quad 293 \\
+\quad 2290 \\
\text { cRGB }\end{array}$ \\
\hline
\end{tabular}

TAIA_SLESABR

NUMBER OF STATISNS: 6

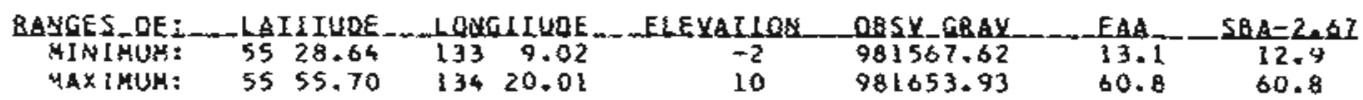

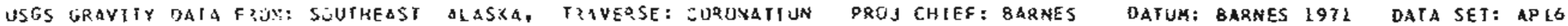

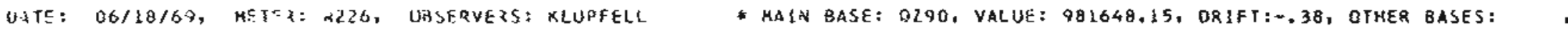

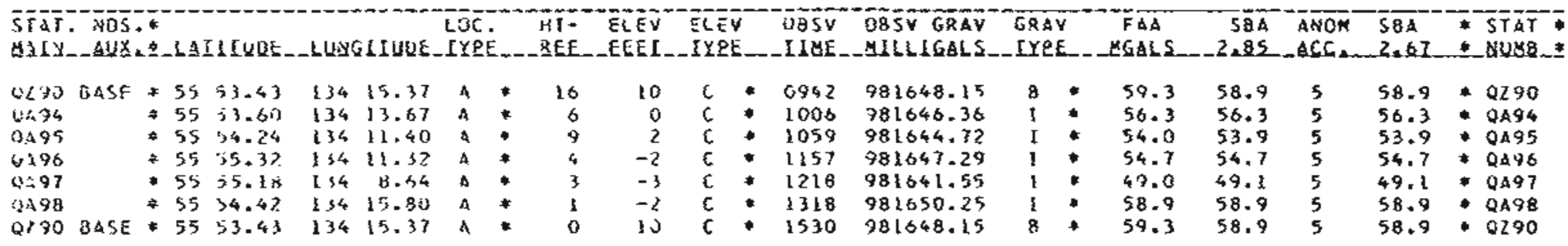

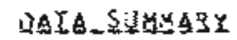

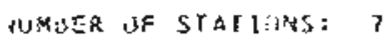

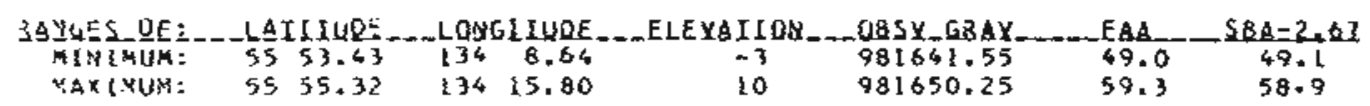

USGS GRAVITY DATA FRDM: SOUTHEAST ALASKA, TRAVERSE: CRAIG-PET PROS CHIEF: GARNES DATUA: BARNES 1971 OATA SET: APL7

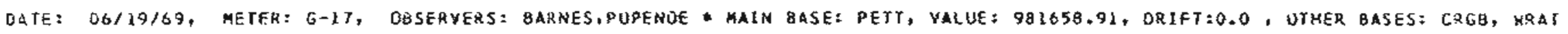

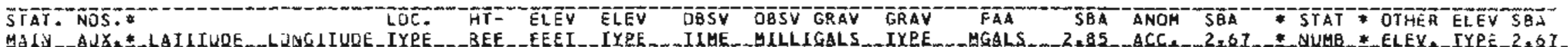

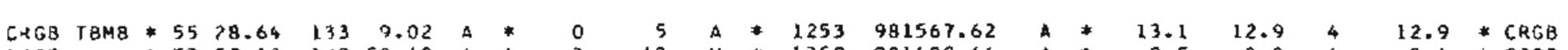

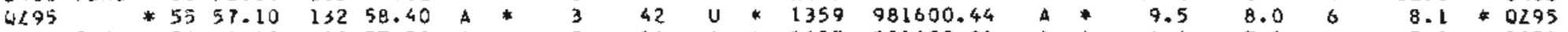

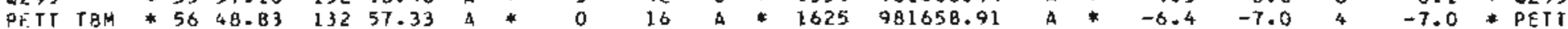

DAIA SUUHABY

NUMERE JF STATIT,YS:

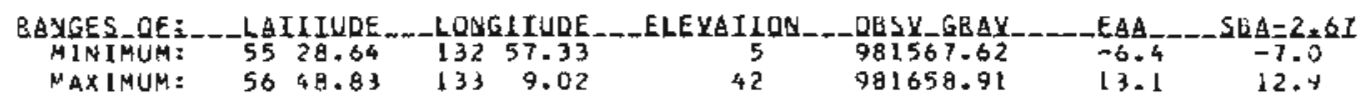




\begin{tabular}{|c|c|c|c|c|c|c|c|c|c|c|c|c|c|c|c|c|c|c|c|c|}
\hline $\begin{array}{l}\text { SIAT. } \\
\text { UALA. }\end{array}$ & $\begin{array}{r}\mathrm{M} \overline{0} \\
-\mathrm{Au}\end{array}$ & & & & & 24 & & BEE. & EEI & $\begin{array}{l}\bar{E} \bar{E} \\
I Y E\end{array}$ & & $\begin{array}{l}\text { OSSY } \\
\text { ILEE. }\end{array}$ & $\begin{array}{l}\text { OAS GAY } \\
\text { MLL LGALS. }\end{array}$ & $6 \pi$ & & $\begin{array}{l}\text { FAA } \\
\text { YGLS }\end{array}$ & $\begin{array}{r}58 . \\
2.85\end{array}$ & $\begin{array}{l}\text { ANOA } \\
\text { ALE. }\end{array}$ & $\begin{array}{c}58 . \\
2=6.7\end{array}$ & 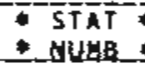 \\
\hline RAT & BASE & +56 & 27.95 & 132 & 22.71 & 4 & + & 18 & 12 & c & $*$ & 1042 & 981615.64 & A & $\bullet$ & -21.1 & -21.6 & 4 & -21.6 & - WAA T \\
\hline IFAB & 7846 & * 56 & & 132 & 23.20 & 4 & + & 0 & 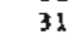 & A & $*$ & 1103 & 901614.51 & 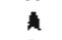 & 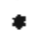 & $-21 \cdot 7$ & -22.9 & 4 & $-22 . a$ & - MAAB \\
\hline WRAP & P.O. & +56 & 28.65 & 132 & 23.19 & A & - & 44 & 37 & A & $\bullet$ & 1111 & 981614.25 & B & 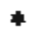 & $-21 \cdot 4$ & -22.6 & 4 & -22.7 & - HAAP \\
\hline HRAT & BASE & $\neq 56$ & 27.95 & 132 & 22.71 & A & * & 0 & 12 & $c$ & * & 1215 & 901615.63 & B & $\bullet$ & -21.2 & -22.6 & 4 & $-2 L \cdot 6$ & * HRAT \\
\hline NENT & $A=P$ & *56 & 29.12 & $1 ; 2$ & 22.64 & A & $*$ & 0 & 60 & c & $*$ & 1250 & 901615.44 & B & $\star$ & -18.5 & -20.6 & 4 & -20.5 & * MRNT \\
\hline $5 \times 96$ & IVEN & +56 & 20.89 & 132 & 22.61 & A & : & 17 & 11 & E & $\star$ & 1407 & 991602.40 & $\mathbf{9}$ & 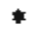 & -24.7 & -25.1 & 4 & -25.0 & * $5 \times 96$ \\
\hline GY 1 & $/ \mathrm{SAT}$ & * 56 & 22.90 & 132 & 8.17 & A & $*$ & 18 & 13 & C & * & 1427 & 981601.59 & 0 & 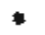 & -28.1 & -28.6 & 4 & -28.5 & * or 1 \\
\hline $4 \times 2$ & & +56 & 22.03 & 132 & 5.89 & A & $*$ & 3 & -1 & C & $*$ & 1447 & 982598.60 & $\mathrm{D}$ & $\star$ & -31.2 & -31.2 & 4 & -31.2 & * or 2 \\
\hline QY 3 & & - 56 & 22.50 & 152 & 3.61 & A & - & $z$ & -2 & $t$ & $\div$ & 1500 & 981597.18 & 0 & * & -33.5 & -33.4 & 4 & -33.4 & - or 3 \\
\hline OY 4 & & * $5 b$ & $21 \cdot 25$ & 132 & 2.39 & A & - & 2 & -1 & $t$ & $\bullet$ & 1512 & 901594.80 & 0 & $\bullet$ & -33.9 & -33.9 & 4 & & - or 4 \\
\hline or 5 & & $* 56$ & 21.88 & 132 & 0.40 & A & * & 1 & -2 & $c$ & $*$ & 1521 & 981597.50 & 0 & $\star$ & $-32 \cdot 2$ & -32.1 & 4 & $-3.2=1$ & * or 5 \\
\hline OY 6 & & * 56 & 22.89 & 131 & 57.11 & A & - & 2 & 1 & $c$ & $*$ & 1602 & 20.9 .8 & 0 & $\bullet$ & -39.6 & -39.8 & 4 & -39.8 & - OY 6 \\
\hline Q4 7 & & - 56 & 20.49 & 131 & 59.32 & 4 & $*$ & 2 & 2 & c & - & 1627 & 9.05 & 0 & * & -38.3 & $-3 a \cdot 4$ & 4 & -38.4 & * ar 7 \\
\hline $0 \times 8$ & /CAP & $* 56$ & 18.99 & 131 & 59.90 & A & + & 6 & 7 & c & $*$ & 1645 & 0.83 & D & 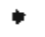 & -34.0 & -34.2 & 4 & -34.2 & * or $\mathrm{a}$ \\
\hline UY 9 & & $* 56$ & 17.75 & 131 & 58.20 & A & $*$ & 4 & 6 & c & $*$ & 1705 & $9 a 1$ & 0 & * & -36.6 & -36.8 & 4 & -36.8 & * or 9 \\
\hline QY 10 & & $\Rightarrow 56$ & 16.24 & 131 & 57.04 & A & $*$ & 3 & 5 & c & $*$ & 1710 & 991 & 0 & $\bullet$ & -43.1 & $-43 \cdot 3$ & 4 & $-43 \cdot 3$ & * ar10 \\
\hline QY11 & & $* 56$ & 14.66 & 131 & 57.42 & A & * & 2 & 5 & [ & * & 1730 & $98 L$ & $D$ & $*$ & -34.4 & -34.6 & 4 & -34.6 & * QYII \\
\hline $0 \times 12$ & 1843 & * 56 & 13.58 & 131 & 55.80 & A & * & B & 12 & c & * & 1755 & 981 & 0 & $\star$ & $-32 \cdot 3$ & -32.7 & 4 & -32.7 & + orl2 \\
\hline $0 \times 13$ & & - 56 & 13.79 & 131 & 52.60 & $\mathbf{A}$ & * & 1 & 6 & c & - & 1815 & 0.73 & 0 & - & -36.9 & -37.2 & 6 & $-37 \cdot 1$ & * OYL3 \\
\hline $5 \div 79$ & QASE & - 56 & 22.21 & 131 & 52.23 & a & - & 21 & 16 & $c$ & $*$ & 1830 & $9 \mathrm{at}$ & a & - & -34.9 & -35.5 & 4 & -35.5 & - 5YT9 \\
\hline $0 \times 14$ & & * 56 & 11.72 & 131 & 57.09 & A & 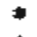 & 1 & 6 & c & * & 1650 & 0.62 & 0 & - & -36.2 & -36.4 & + & -36.4 & - orit \\
\hline $0 \div 15$ & & - 56 & 11.68 & 131 & 59.81 & 4 & * & 1 & 6 & $c$ & - & 1902 & 981 & 0 & $*$ & -27.1 & -2 & 4 & -27.3 & - OY15 \\
\hline QY16 & & - 56 & 13.17 & 132 & 2.10 & 4 & - & l & 6 & c & $*$ & 1020 & 981 & 0 & - & -25.6 & -25.8 & 4 & -25.8 & - aris \\
\hline $0 \times 17$ & & 50 & 10.05 & 132 & 0.90 & 4 & $*$ & 1 & 6 & c & $*$ & 1940 & 981587.45 & 0 & $\star$ & -25.0 & -25.2 & 4 & -25.2 & * Qrit \\
\hline $0 \times 18$ & & $\Rightarrow 56$ & 5.30 & 132 & -14 & A & $*$ & 3 & a & c & $*$ & 2020 & 9015 & $\mathrm{D}$ & $*$ & -19.1 & -19.4 & 4 & & - OYIB \\
\hline QY14 & & * 56 & 3.53 & 132 & 3.60 & A & * & 2 & 7 & c & $*$ & 2036 & 2.89 & 0 & $*$ & -20.4 & -20.6 & 4 & -20.6 & * QYI9 \\
\hline QY20 & & \& 56 & 2.73 & 132 & 6.59 & A & * & 1 & 5 & 6 & * & 2051 & 72 & D & $*$ & -16.6 & -16.8 & 4 & -16.8 & * OYZO \\
\hline or 21 & ТВМ2 & $\neq 56$ & 3.77 & 132 & 12.20 & A & $*$ & 6 & 9 & t & $\bullet$ & 2115 & 991591.83 & D & $*$ & -11.6 & -11.9 & 4 & -11.9 & * QYZI \\
\hline $0 \times 22$ & & -56 & 7.75 & 132 & 0.18 & A & * & 3 & 5 & E & * & 2150 & 981596.49 & 0 & $*$ & $-12 \cdot 9$ & $-13 \cdot 0$ & 4 & -13.0 & * OY22 \\
\hline $0 \times 23$ & & -56 & 9.12 & 232 & 6.13 & d & * & 1 & 2 & $c$ & + & 2210 & 98150 & D & $\bullet$ & -16.9 & -17.0 & 4 & -1 & - OrZ3 \\
\hline QY24 & & - 56 & 9.55 & 132 & 10.91 & $A$ & $*$ & 1 & 2 & $c$ & + & 2228 & $9 a$ & $\mathrm{~B}$ & $\bullet$ & -11.7 & $-11, B$ & 4 & .8 & - OY24 \\
\hline GY 25 & BASE & - 56 & $2 L .07$ & $1 \pm 2$ & 12.72 & A & - & 13 & 13 & 6 & * & 2243 & 981601.77 & $h$ & $\bullet$ & -11.4 & -11.9 & 4 & -11.9 & - QYZs \\
\hline$H \times A B$ & TBMS & $\neq 50$ & 29.12 & 132 & 22.64 & a & 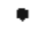 & 0 & 31 & c & $\bullet$ & 2418 & 981614.51 & A & $*$ & -22.1 & -23.2 & 4 & -23.2 & - MRAB \\
\hline
\end{tabular}

DAIA SUHHAQY

NUMBEK UF STATISNS: 33

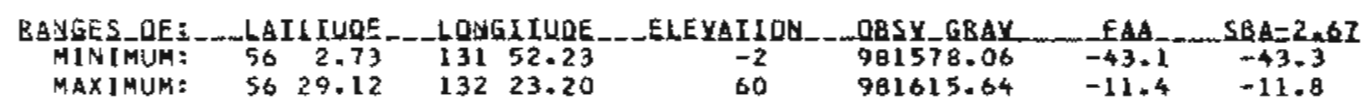


USGS GRAVITY DATA FROM: SOUTHEAST ALASKA, TRAVERSF: LIMOUIA ST PROS CMIEF; BARHES DATUA: BARNES IGTL OATA SET: APZI

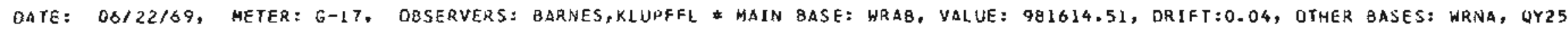

\begin{tabular}{|c|c|c|c|c|c|c|c|c|c|c|c|c|c|c|c|c|c|c|}
\hline $\begin{array}{l}\text { Stat. } \\
\text { UALY. }\end{array}$ & 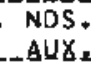 & 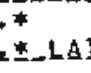 & in & & & & & & EV & 50 & $\begin{array}{l}\text { UBSV } \\
\text { IIUE }\end{array}$ & $\begin{array}{l}\text { QBSV GRAV } \\
\text { WLLIGALS }\end{array}$ & & $\begin{array}{l}\text { FAA } \\
\text { UGALS }\end{array}$ & $\begin{array}{r}\text { SBA } \\
2.85\end{array}$ & $\begin{array}{l}\text { ANOM } \\
A C E \text {. }\end{array}$ & $\begin{array}{l}58 . \\
2.67\end{array}$ & $\begin{array}{r}\text { STAT } \\
\text { * מلta日-4 }\end{array}$ \\
\hline HRAB & TBHO & 56 & 28.85 & 132 & 23.20 & A & & 0 & 31 & A & 1103 & 81614.51 & $A$ & -21.7 & -22.9 & 4 & -22.8 & NA $A B$ \\
\hline HRAP & & * 56 & & 132 & & A & $T$ & 0 & 3 & A & & & B & & & 4 & & - MRAP \\
\hline WRAT & BASE & * 56 & 27.95 & 132 & 22.71 & $\mathbf{A}$ & * & 21 & 19 & $c$ & 1144 & 901615.64 & 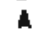 & $-20+5$ & -2 & 4 & -21.1 & WRAT \\
\hline WRNA & $B A S E$ & * 56 & 25.47 & 132 & 21.50 & $\mathrm{~A}$ & * & 11 & 8 & C & 1227 & 981611 & A & $-22 \cdot 3$ & -22 & 4 & -22.5 & - MRMA \\
\hline Q 26 & & - 56 & $23.6 \mathrm{i}$ & 132 & $20.5 \mathrm{I}$ & A & $*$ & $i$ & -5 & $c$ & 1243 & $981613.4 \mathrm{a}$ & 0 & -18.9 & -18.7 & 4 & -18.7 & - बY26 \\
\hline QY $2 T$ & & $* 56$ & 22.17 & 132 & $21-69$ & $\hat{A}$ & 4 & 3 & -3 & C & 1257 & 981615.66 & 0 & $* \quad-14.5$ & -14.4 & 4 & -14.4 & - QYZT \\
\hline $\operatorname{ar} 28$ & & * 56 & 20.39 & 132 & 5 & $\mathbf{A}$ & 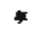 & 3 & -3 & $c$ & 13 & 98 & 0 & -13.7 & -13 & 4 & -13.6 & $*$ \\
\hline or 29 & & - 56 & 38.32 & 132 & 20.01 & A & $*$ & 16 & 9 & $\mathrm{C}$ & 13 & 1.2 & D & -12.4 & -12 & 4 & -12.7 & 29 \\
\hline Or 30 & & $* 56$ & 16.11 & 132 & 20.70 & $\mathbf{A}$ & $*$ & 3 & -4 & $c$ & 13 & 981 & $\mathrm{D}$ & $-13,4$ & -13. & 4 & -13.3 & 30 \\
\hline QY 31 & & * 56 & 14.29 & 132 & 19.80 & A & * & 2 & -4 & 6 & 14 & 981 & 0 & -8.2 & -8 & 4 & $-8,1$ & $=$ \\
\hline by 32 & & * 56 & 13.22 & 132 & 17.50 & $A$ & * & 2 & -4 & $c$ & 14 & 98 & 0 & -9.7 & -9 & 4 & -9.6 & - 0r32 \\
\hline $0 \times 33$ & & $\neq 56$ & 12.23 & 132 & A & A & $*$ & 5 & -1 & $c$ & 14 & 9 & 0 & -10.3 & -10 & 4 & -10.3 & 33 \\
\hline QY25 & BASE & -56 & 11.07 & 132 & 12.74 & $A$ & $*$ & 18 & 13 & c & 15 & 981 & $\mathrm{~B}$ & -11.4 & -11 & 4 & -11.9 & -0 \\
\hline OY 34 & 5044 & +56 & 11.36 & 132 & 19.22 & 4 & * & 12 & 10 & $c$ & 16 & $98161 \mathrm{l}$ & 0 & -2.9 & -1 & 4 & -3.2 & - ar34 \\
\hline QY35 & & 56 & 12.78 & 132 & 20.83 & $A$ & $*$ & I & 1 & $c$ & * 1708 & $9 B L$ & 0 & -4.4 & -4 & 4 & -4.4 & * ar3s \\
\hline Qr36 & & $* 56$ & 12.33 & 132 & 23.76 & $A$ & * & 5 & 6 & $c$ & in & 98 & 0 & -9.0 & -8 & 4 & -8.2 & 36 \\
\hline $0 \times 37$ & & $\neq 56$ & 11.27 & 132 & 75 & $\mathbf{A}$ & * & 2 & 3 & $t$ & 13 & 9 & $D$ & -11.7 & -11 & 4 & -11.8 & 37 \\
\hline DY 38 & & $\begin{array}{l}-56 \\
\end{array}$ & 11.60 & 132 & 30.33 & $\mathbf{A}$ & $\neq$ & 2 & 4 & $t$ & 18 & 981617.20 & D & 2.4 & 2.3 & 4 & 2.3 & +8 \\
\hline $0 \times 39$ & & $\div 56$ & 12.44 & 132 & 27.27 & $A$ & $\#$ & 2 & 5 & $c$ & 18 & $96 \mathrm{I}$ & $a$ & -9. & & 4 & -9.4 & $*$ \\
\hline OY 40 & & -56 & 14.44 & 132 & 23.71 & $\mathbf{A}$ & $*$ & 1 & 5 & $\mathrm{c}$ & 1844 & 981 & 0 & -5.4 & -5 & 6 & -5.6 & - orao \\
\hline $0 \times 41$ & /XON & $\Rightarrow 56$ & 15.62 & 132 & 22.73 & 4 & * & 3 & 7 & $c$ & 1856 & 901611.33 & 0 & -8.9 & & 4 & -9.0 & * QY4l \\
\hline bY 42 & & * 56 & 17.03 & 132 & 23.58 & $\mathbf{A}$ & * & 1 & 6 & $\mathrm{c}$ & 1912 & 981617.04 & 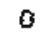 & -5.1 & -5 & 4 & -5.3 & - 0r42 \\
\hline 9443 & & +56 & 19.34 & 132 & 23.71 & $\mathrm{~A}$ & * & $i$ & 6 & $c$ & 1930 & 981 & $\mathrm{D}$ & -10.8 & -11 & 4 & $-1 \mathbf{i} .0$ & * QY43 \\
\hline $0 \times 44$ & /PAS & * 50 & 20.90 & 132 & 25.10 & A & * & 5 & 10 & $\mathrm{c}$ & 1947 & 981 & $\mathrm{D}$ & -12.5 & -12 & 4 & -1 & - OY44 \\
\hline 0445 & & - 56 & 20.43 & 132 & 28.73 & $\mathbf{A}$ & * & 3 & $\theta$ & $c$ & 2005 & 981614. & 0 & -12.3 & -12 & 4 & -12.5 & - oras \\
\hline 9446 & & $\approx 56$ & 19.67 & $1 \leq 2$ & 32.51 & $\vec{A}$ & $\star$ & 1 & 6 & $c$ & 2021 & 901622. & 0 & -3.5 & -3 & 4 & -3.7 & - Or46 \\
\hline $0 \times 47$ & & & 17.44 & 132 & 34.50 & $\mathbf{A}$ & * & 1 & 6 & [ & 301 & 961624.85 & 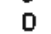 & 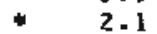 & 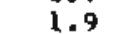 & 4 & 1.9 & * QY47 \\
\hline QY48 & & * 56 & 21.33 & 132 & 33.19 & A & * & 3 & B & $\mathrm{C}$ & 2103 & 981621.89 & 0 & -6.1 & & 4 & -6.3 & * Or48 \\
\hline 0449 & & - & 22.46 & 132 & 27.19 & $\mathbf{A}$ & \# & 2 & 6 & c & 2125 & 981615 & 0 & -14.4 & -14 & 4 & -14.6 & - OY49 \\
\hline WRNA & BASE & $* 5$ & 25.47 & 132 & 21.50 & $A$ & * & 2 & 6 & $c$ & 2150 & 981 & A & -22.3 & -22 & 4 & -22.5 & * ARMA \\
\hline HNAT & BASE & * 5.6 & 27.95 & 132 & 22.71 & $\mathbf{A}$ & * & 9 & 12 & $c$ & 2212 & 981615.64 & A & -21.1 & & 4 & -21.6 & - WRAT \\
\hline WRAB & TBMG & $\approx 56$ & 28.05 & 132 & 23.20 & A & * & 0 & 31 & A & 2235 & 981614.51 & A & $-21 \cdot 7$ & & 4 & -22.0 & - WRAB \\
\hline
\end{tabular}

QALA_SUGHASX

NUMBER OF STAIIUAS: 37
BAUGF5 OE: MINMUM: MAXIMUM:

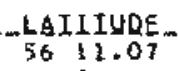

56
5628.07
-LONGI TUDE

$\begin{array}{ll}132 & 12.74 \\ 132 & 34.50\end{array}$
FLEYAIIUN

$-5$

37
OBSY GRA

981601.77
981624.95
$=2 \frac{E A}{3}$
$-5 B A=2.67$

$-22.8$ 


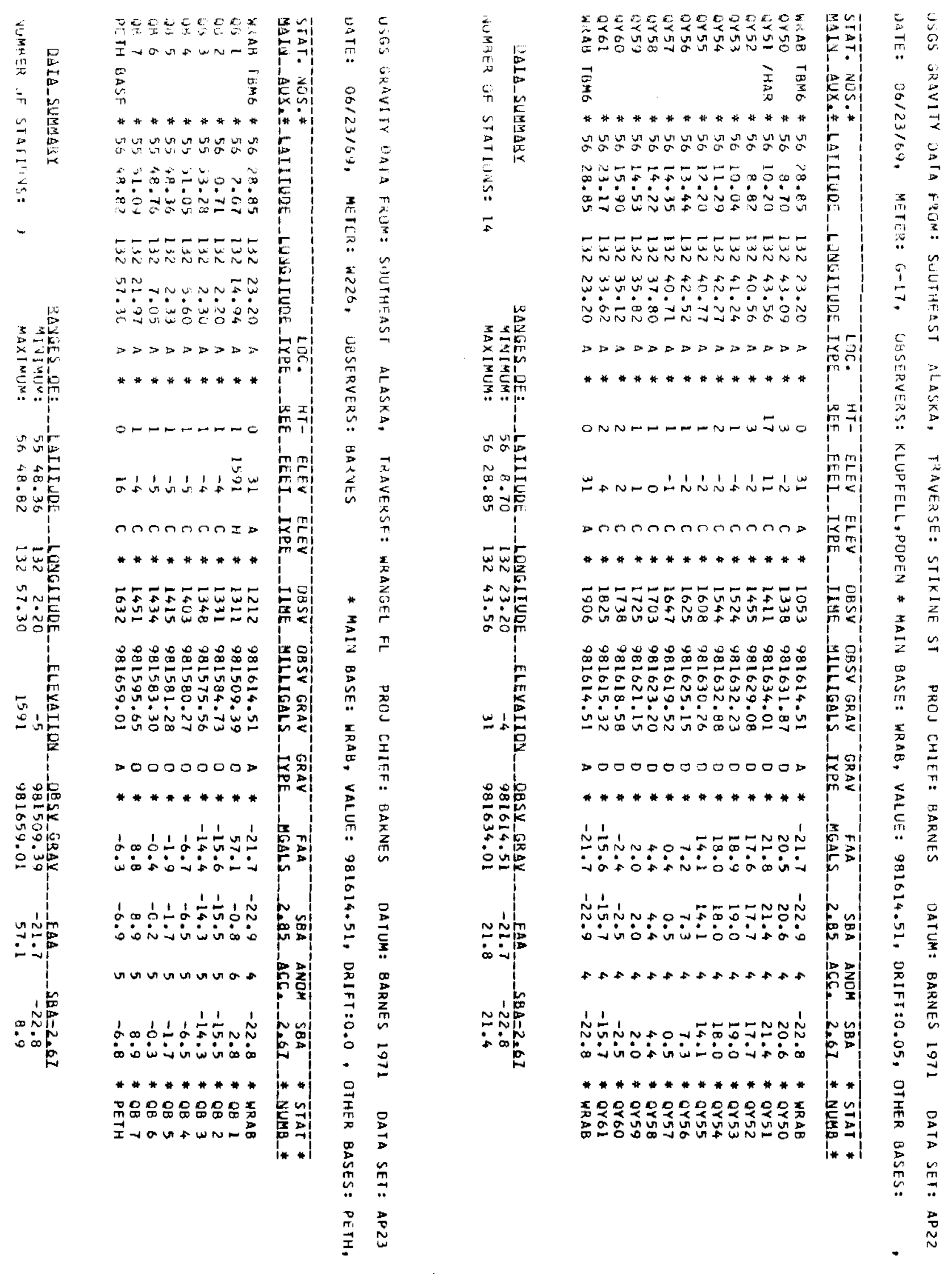


USGS GRAVITY DAIA FRDM: SOUTHFAST ALASKA, TRAVERSE: PETH-BAKER

PAOJ CHIEF: HARNES

DATUM: BARNES 1971 DATA SET: AP24

DUTE: D6/24/69, MEIER: W226, OESERVERS: HARNES

* main gase: 5X90, value: 981653.30 , DRIFT:0.13, OTHER bases: bakr. Peth

\begin{tabular}{|c|c|c|c|c|c|c|c|c|c|c|c|c|c|c|c|c|c|c|c|}
\hline $\begin{array}{l}\text { STAT. } \\
\text { SALY }\end{array}$ & $=N$ & & & & & Li & & & & & & LE & $\begin{array}{l}085 V G A \\
\text { CILLLGAL }\end{array}$ & & $\overline{F A A}$ & $\begin{array}{r}58 \bar{A} \\
2.85\end{array}$ & AN & $\begin{array}{r}5 \mathrm{aA} \\
-2.6\end{array}$ & $\begin{array}{r}\text { STAT } \\
-\quad \text { NNLHB }\end{array}$ \\
\hline$P \leq E H$ & BASE & +50 & 49.82 & 132 & 57.30 & A & - & 0 & 16 & $A$ & $*$ & 1322 & 981659.01 & $\Delta$ & -8.3 & -6.9 & 4 & -6.8 & - PETH \\
\hline $5 \times 90$ & TBA] & 15 & 27.80 & 133 & 5.90 & $A$ & $\star$ & 8 & 7 & $\mathrm{c}$ & $\star$ & 1745 & 981653.30 & 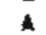 & 16.3 & & 4 & 16.0 & - $5 \times 90$ \\
\hline $5 \times 2$ & BASE & $=5$ & 20.20 & 133 & 16.29 & $\vec{A}$ & $*$ & 12 & 13 & c & * & 1045 & 981643.34 & $\mathrm{~g}$ & 17.4 & 16.9 & 4 & 47.0 & - $5 V_{2}$ \\
\hline 030 & & $* 5$ & 18.34 & 133 & 18.67 & A & $*$ & l & 3 & $\mathrm{C}$ & - & 1903 & 981642.63 & 0 & 17.7 & & 5 & 17.6 & - 0906 \\
\hline $930 \%$ & & 45 & 16.80 & 133 & 18.26 & $A$ & * & i & 4 & $c$ & * & 1920 & 981639.43 & 0 & 4 & & 5 & 17.3 & - 0809 \\
\hline 9910 & & 45 & 16.22 & 133 & 20.64 & A & * & l & 4 & $c$ & * & 1937 & 981634.06 & 0 & 13.4 & $i$ & 5 & 13.3 & - OA10 \\
\hline $4 \times 11$ & & -5 & 17.50 & Is 3 & 20.19 & $A$ & * & i & 5 & c & \# & 1953 & 98.1638 .91 & 0 & 16 & & 5 & 15.8 & - QB I l \\
\hline get 2 & & $\neq 5$ & 18,90 & 133 & 20.68 & $h$ & $*$ & 2 & $s$ & $\mathrm{c}$ & * & 2008 & 981639.66 & 0 & 14 & & 5 & 44.7 & - Q812 \\
\hline 01313 & & -5 & 19.91 & 133 & 18.84 & A & $\bullet$ & L & 6 & $c$ & $*$ & 2027 & 981642.33 & 0 & 16.2 & 15 & 5 & 15.9 & - 0813 \\
\hline 0114 & & $* 50$ & 20.41 & 133 & $21 \cdot 34$ & $A$ & $*$ & 2 & 7 & $c$ & + & 2049 & 981640.70 & 0 & 13.9 & & 5 & 13.7 & - 9614 \\
\hline 0015 & & $* 5$ & 20.27 & 133 & 24.26 & $A$ & $\star$ & 2 & 7 & C & $\star$ & 2103 & 901641.05 & 0 & 14.5 & & 5 & 14.2 & - OE 15 \\
\hline 0316 & & 45 & 20.00 & 133 & 26.79 & A & * & 4 & 10 & $\mathrm{c}$ & $*$ & 2120 & 981639.22 & o & 13.3 & 9 & 5 & 12.9 & - abio \\
\hline Jol 17 & 1510 & +5 & 20.57 & 133 & 29.37 & $A$ & - & 9 & 34 & $\mathrm{C}$ & $*$ & 2138 & 981638.40 & 0 & 11.9 & & 5 & 11.4 & - O817 \\
\hline 18 & & $* 5$ & 20.30 & 133 & $32+1 \mathrm{~B}$ & A & * & 3 & 7 & $c$ & $*$ & 2202 & 981637.83 & 0 & i1 & & 5 & 11.0 & - QBIB \\
\hline 0419 & & $* 5$ & $21 \cdot 14$ & 133 & 34.23 & A & $*$ & l & 5 & $c$ & \# & 2220 & $98163 \mathrm{~B} .99$ & 0 & 11.0 & 10.8 & 5 & 10.8 & - QB 19 \\
\hline 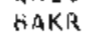 & BAS & $\neq 5$ & 21.25 & 133 & 37.17 & A & $*$ & 0 & $\theta$ & $\mathrm{c}$ & * & 2245 & 981638.15 & $\mathbf{A}$ & 10.3 & 10.0 & 5 & 10.0 & - gaxr \\
\hline
\end{tabular}

QAIA_SUSUABY

NUMBER LF STATIINS: 16

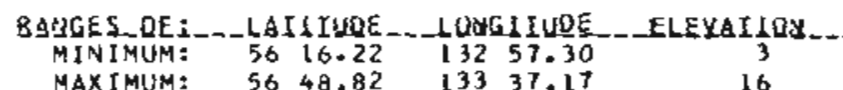

MINIMUM:

MAXIMUM:
13257.30
13337.27

16
981634.66

981659.01

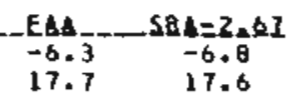


USG5 GRAYLTY DATA FROM: SOUTHEAST ALASKA, TRAVERSE: LEVEL IS W PROJ CHIEF: BARNES DATUM: BARNES 1971 UATA SET: AP25

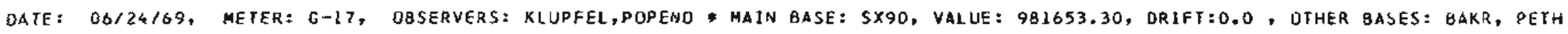

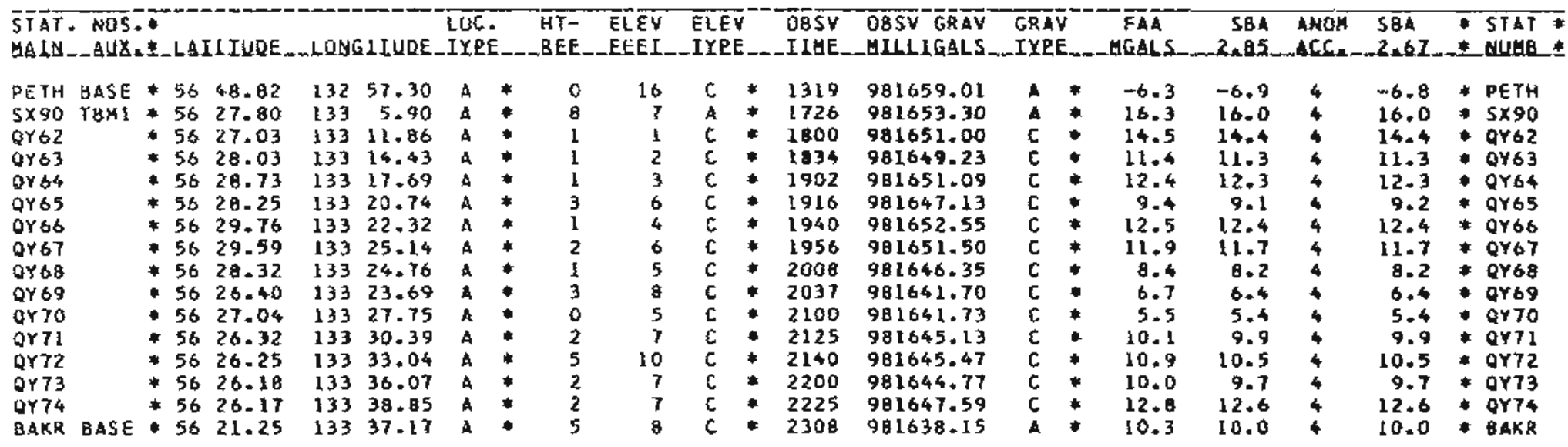

METER G-17 HENT OFF IEMPERATURE SHORTLY BEFORE FINAL READING

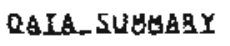

NUMBER OF STATIUNS: 16

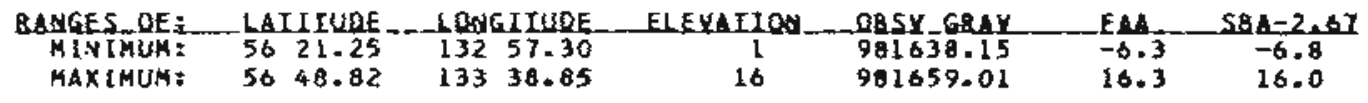


USGS GRAVITY OATA FROM: SUUTHEAST ALASKA, TRAVERSE: 3-MILE ARM PRGJ CHIEF: BARNES

DATUHE BARNES 1971 DATA SET: AP26

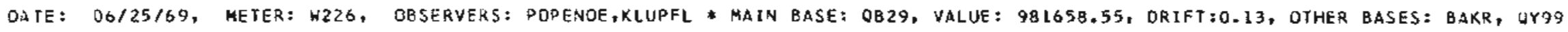

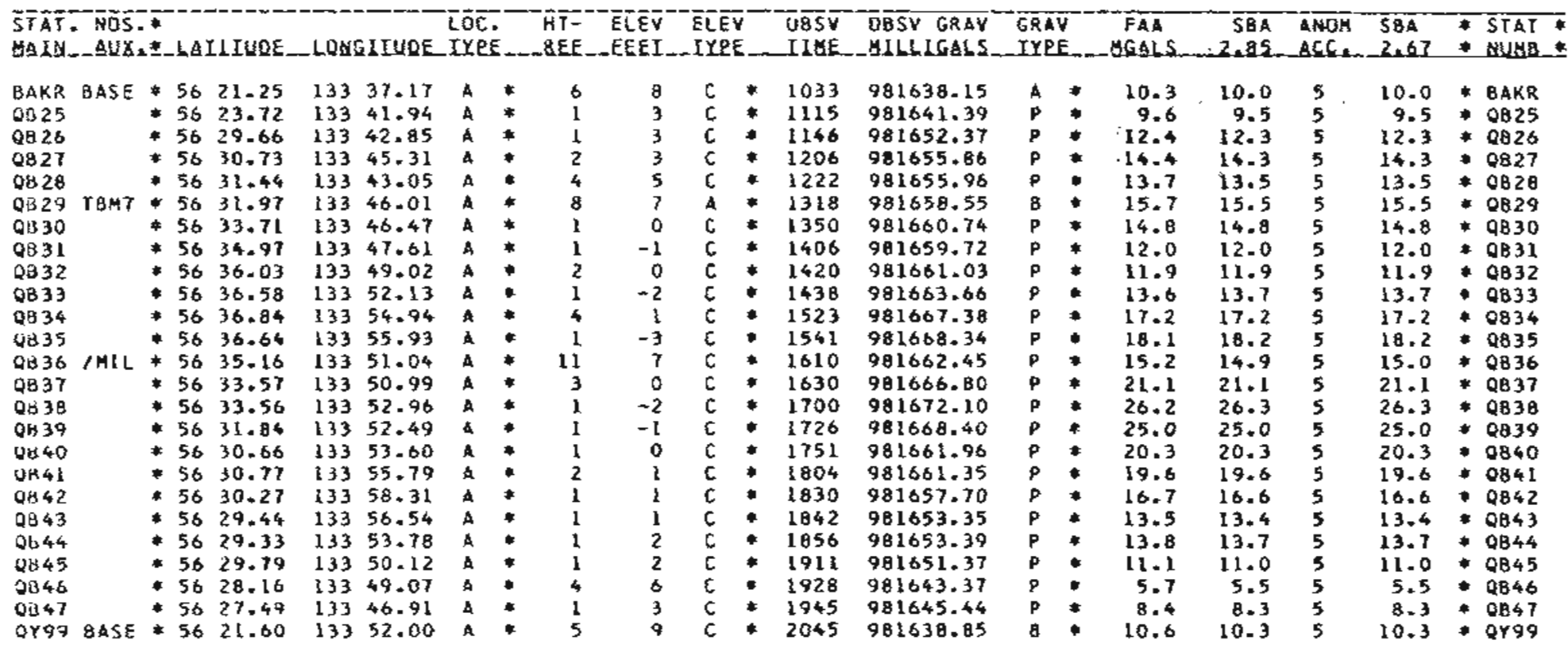


USGS GRAYITY DATA FRUM: SOUTHEAST ALASKA, TRAVERSE: BAKER-REID PROJ CHIEF: BARNES DATUM: BARNES 1971 DATA SET: AP27 DATE: OS/25/69, HETER: GMI7. OBSERYERS: BARES

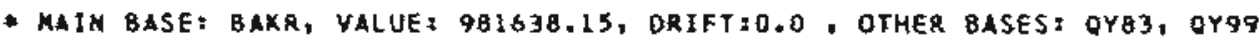

\begin{tabular}{|c|c|c|c|c|c|c|c|c|c|c|c|c|c|c|c|c|c|c|c|c|}
\hline TAL & & & & & & & & & $v$ & & & $\begin{array}{l}\text { OBSY } \\
\text { ILUE. }\end{array}$ & $\begin{array}{l}\text { OBSY GRAV } \\
\text {-HILLIGALS. }\end{array}$ & $\bar{G}$ & & $\begin{array}{l}\text { FAA } \\
\text { OGALS }\end{array}$ & $\begin{array}{r}5 B \bar{A} \\
2.95\end{array}$ & NO & $\begin{array}{l}58 . \\
2.67\end{array}$ & $\begin{array}{l}-5 T 1 \\
+\quad 4 u\end{array}$ \\
\hline 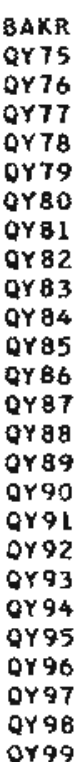 & $\begin{array}{l}\text { BASE } \\
\text { IB.43 }\end{array}$ & 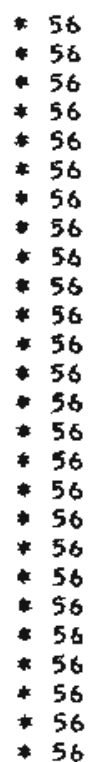 & $\begin{array}{l}21.25 \\
21.13 \\
19.98 \\
18.95 \\
18.44 \\
17.19 \\
19.74 \\
19.33 \\
17.12 \\
15.82 \\
15.57 \\
15.48 \\
16.68 \\
17.16 \\
16.43 \\
15.12 \\
18.64 \\
18.75 \\
20.29 \\
21.37 \\
19.92 \\
17.92 \\
16.50 \\
19.06 \\
21.00 \\
21.60\end{array}$ & $\begin{array}{l}133 \\
133 \\
133 \\
133 \\
133 \\
133 \\
133 \\
133 \\
133 \\
133 \\
133 \\
133 \\
133 \\
133 \\
133 \\
133 \\
133 \\
133 \\
133 \\
133 \\
133 \\
133 \\
133 \\
133 \\
133 \\
133\end{array}$ & $\begin{array}{l}37.67 \\
37.38 \\
36.63 \\
36.68 \\
36.07 \\
35.66 \\
39.12 \\
39.53 \\
39.31 \\
38.34 \\
51.37 \\
54.37 \\
55.09 \\
55.68 \\
58.69 \\
59.17 \\
57.19 \\
59.50 \\
58.74 \\
58.87 \\
56.10 \\
53.37 \\
52.48 \\
50.57 \\
50.97 \\
52.00\end{array}$ & $\begin{array}{l}A \\
A \\
A\end{array}$ & 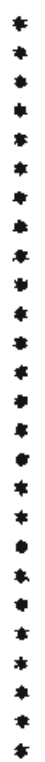 & $\begin{array}{r}6 \\
5 \\
1 \\
1 \\
6 \\
2 \\
5 \\
4 \\
2 \\
1 \\
2 \\
1 \\
2 \\
12 \\
1 \\
8 \\
1 \\
1 \\
2 \\
3 \\
2 \\
1 \\
2 \\
3 \\
2 \\
4\end{array}$ & $\begin{array}{r}8 \\
7 \\
3 \\
2 \\
7 \\
2 \\
4 \\
2 \\
0 \\
8 \\
-1 \\
-2 \\
-1 \\
10 \\
0 \\
7 \\
1 \\
1 \\
3 \\
4 \\
4 \\
3 \\
5 \\
6 \\
6 \\
9\end{array}$ & $\begin{array}{l}c \\
1 \\
c \\
c \\
c \\
c \\
c \\
c \\
c \\
1 \\
c \\
c \\
c \\
c \\
c \\
c \\
c \\
c \\
c \\
c \\
c \\
c \\
c \\
c \\
c \\
c\end{array}$ & $\begin{array}{l}* \\
* \\
* \\
* \\
* \\
* \\
* \\
* \\
* \\
* \\
* \\
* \\
* \\
* \\
* \\
* \\
*\end{array}$ & $\begin{array}{l}1041 \\
1103 \\
1141 \\
1233 \\
1241 \\
1254 \\
1347 \\
1402 \\
1417 \\
1455 \\
1600 \\
1640 \\
1455 \\
1735 \\
1752 \\
1810 \\
1832 \\
1947 \\
1901 \\
1913 \\
1928 \\
1945 \\
2003 \\
2019 \\
2036 \\
2051\end{array}$ & $\begin{array}{l}981638.49 \\
981638.05 \\
981834.78 \\
981636.42 \\
981635.62 \\
981634.01 \\
981635.56 \\
981635.49 \\
981635.01 \\
981631.60 \\
981633.42 \\
981633.09 \\
981634.61 \\
981638.42 \\
981640.53 \\
981637.28 \\
981640.21 \\
981640.84 \\
981641.36 \\
981641.90 \\
961634.97 \\
981637.55 \\
981635.30 \\
981635.53 \\
981637.67 \\
981638.85\end{array}$ & $\begin{array}{l}1 \\
D \\
D \\
0 \\
D \\
0 \\
0 \\
0 \\
D \\
A \\
D \\
D \\
D \\
0 \\
0 \\
D \\
D \\
0 \\
D \\
0 \\
0 \\
D \\
D \\
D \\
D \\
0\end{array}$ & 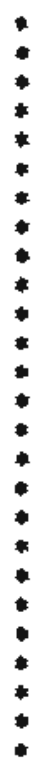 & $\begin{array}{r}10.6 \\
10.3 \\
13.2 \\
11.3 \\
11.6 \\
11.2 \\
9.4 \\
11.1 \\
12.1 \\
11.3 \\
12.6 \\
12.3 \\
12.3 \\
16.4 \\
10.6 \\
17.9 \\
15.3 \\
15.8 \\
14.4 \\
13.5 \\
8.6 \\
13.9 \\
13.7 \\
10.5 \\
10.0 \\
10.6\end{array}$ & $\begin{array}{r}10.3 \\
10.0 \\
8.1 \\
11.3 \\
11.3 \\
11.2 \\
9.3 \\
11.1 \\
12.1 \\
11.0 \\
12.7 \\
12.4 \\
12.3 \\
16.1 \\
18.8 \\
17.6 \\
15.3 \\
15.4 \\
14.3 \\
13.4 \\
8.4 \\
13.1 \\
13.5 \\
10.3 \\
9.8 \\
10.3\end{array}$ & $\begin{array}{l}4 \\
4 \\
4 \\
4 \\
4 \\
4 \\
4 \\
4 \\
4 \\
4 \\
4 \\
4 \\
4 \\
4 \\
4 \\
4 \\
4 \\
4 \\
4 \\
4 \\
4 \\
4 \\
4 \\
4 \\
4 \\
4\end{array}$ & $\begin{array}{l}10.4 \\
10.0 \\
0.1 \\
11.3 \\
11.3 \\
11.2 \\
9.3 \\
11.1 \\
12.1 \\
11.0 \\
12.7 \\
12.4 \\
12.3 \\
16.1 \\
14.6 \\
17.6 \\
15.3 \\
15.8 \\
14.3 \\
13.4 \\
13.5 \\
13.8 \\
13.5 \\
10.3 \\
9.8 \\
10.3\end{array}$ & 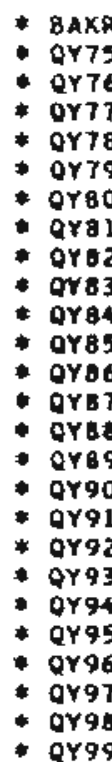 \\
\hline
\end{tabular}

DAIA_SUGHABX

NUHBER OF SIATLONS: 26

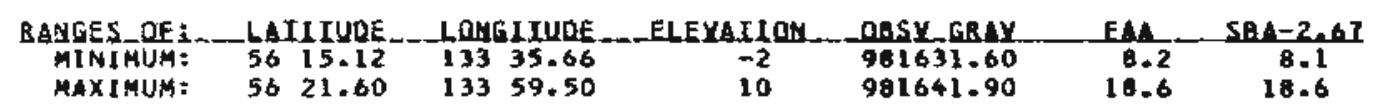




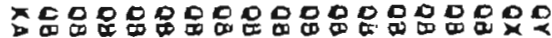

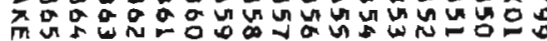

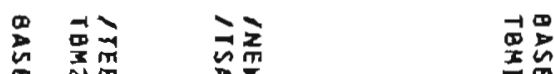

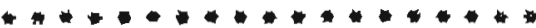

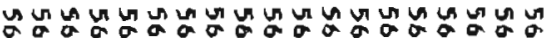

Uค

7

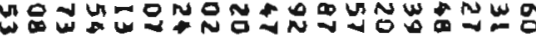

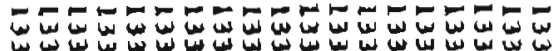

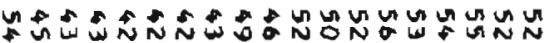

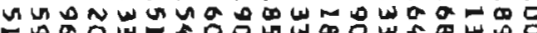

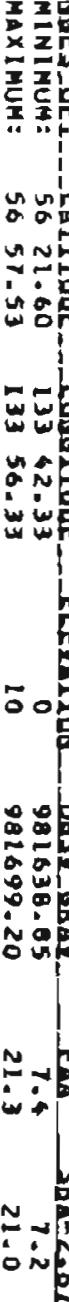

$\triangle D D D D D D D D D D D$

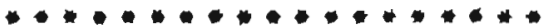

W ש

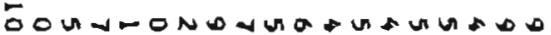

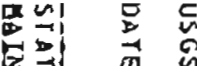

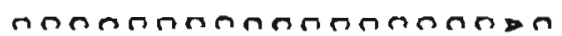

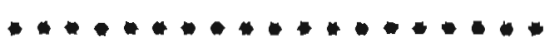

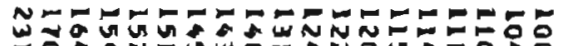

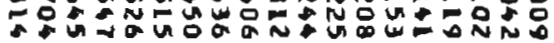

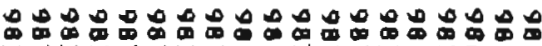

-

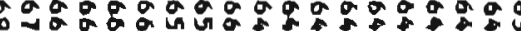

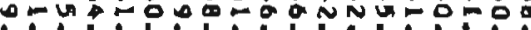

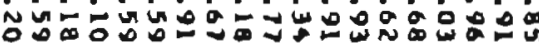

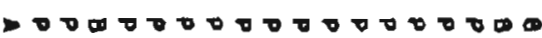

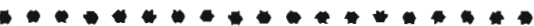

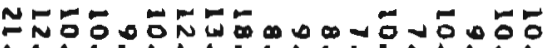

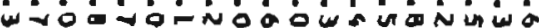

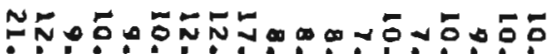

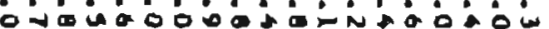

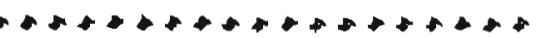

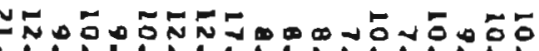

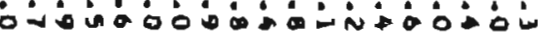

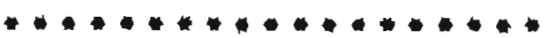

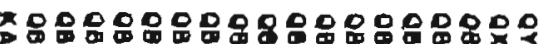

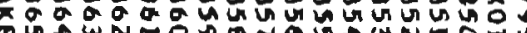


WSGS GRAVITY DATA FROM: SOUTHEAST ALASKA, TRAVERSE: REID-KAKE

PROJ CHIEF: DARNES

DATUH : BARNES 1971

DATA SET: AP 29

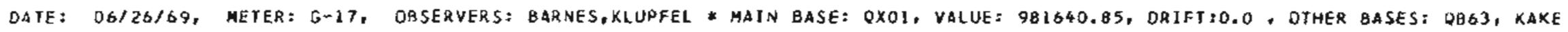

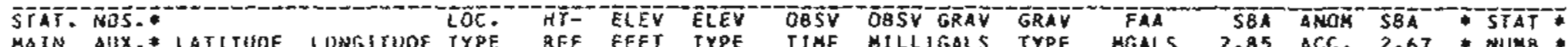

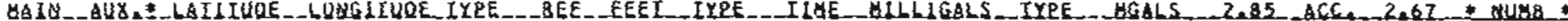

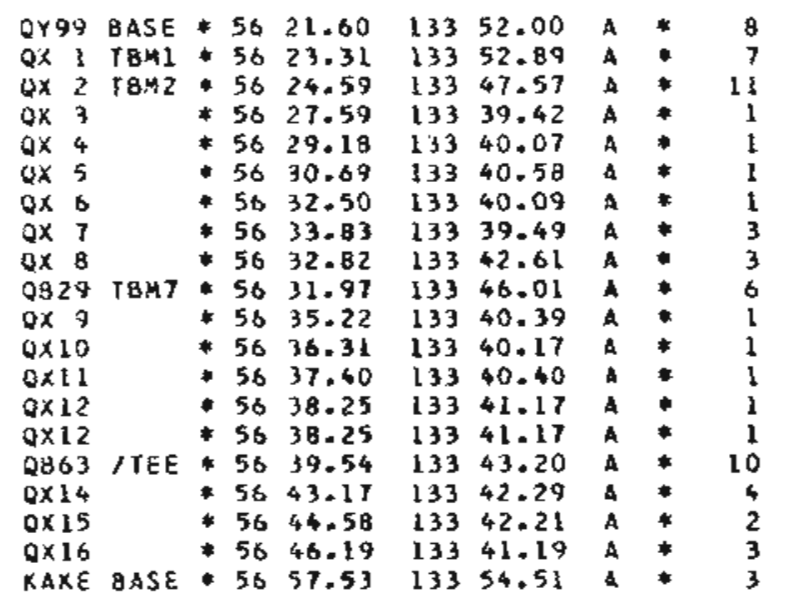

\begin{tabular}{|c|c|c|c|}
\hline 9 & L & 1000 & 981638.95 \\
\hline 9 & A & 1033 & 901640.85 \\
\hline 14 & c & 1115 & 991640.31 \\
\hline 4 & C & 1152 & 981651.52 \\
\hline 4 & 6 & 1211 & 981653.21 \\
\hline 4 & 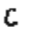 & 1227 & 981654.52 \\
\hline 4 & C & 1242 & 981559.94 \\
\hline 5 & c & 1259 & 981661.35 \\
\hline 5 & c & 1315 & 981659.43 \\
\hline 7 & A & 1335 & 981658.55 \\
\hline 0 & c & 1455 & 981660.71 \\
\hline-1 & $\mathrm{c}$ & 1530 & 981660.22 \\
\hline-1 & 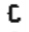 & 1542 & 981660.83 \\
\hline-2 & $c$ & 1557 & 981662.18 \\
\hline-2 & 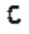 & 1557 & 981662.18 \\
\hline 7 & A & 1617 & 981664.10 \\
\hline I & c & $-\quad$ [ 107 & 981670.97 \\
\hline-1 & c & $\begin{array}{l}-1732 \\
\end{array}$ & 981675,29 \\
\hline 0 & c & 1743 & 981878.38 \\
\hline 10 & $c$ & 2309 & 981699.20 \\
\hline
\end{tabular}

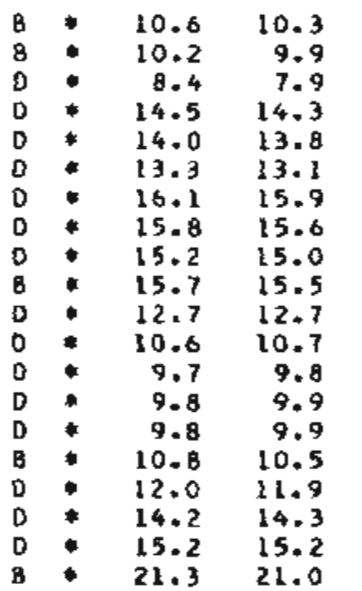

$10.3 * 0 \times 99$
$9.9 * 0 \times 1$
$7.9 * 0 \times 2$
$14.3 * 0 \times 3$
$13.8 * 0 \times 4$
$13.1 * 0 \times 5$
$15.9 * 0 \times 6$
$15.6 * 0 \times 7$
$15.1 * 0 \times 8$
$15.5: 0829$
$12.7: 0 \times 9$
$10.7 * 0 \times 10$
$9.6 * 0 \times 11$
$9.9 * 0 \times 12$
$9.9 * 0 \times 12$
$10.5 * 0863$
$11.9 * 0 \times 14$
$14.3 * 0 \times 15$
$15.2 * 0 \times 16$
$21.0 * 0 \times 1$

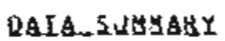

NUMBER DF STATIJNS: 20

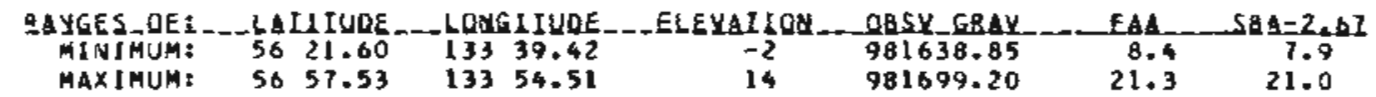


USGS GRAWITY DATA FRGM: SDUFHEAST ALASRA, TRAVERSE: HW KUIU IS PROJ CHJEF: BARNES DATUH: BARNES 1971 OATA SET: APJO DHFE : G6/27/69, METER: G-17, DBSERYERS: POPENOF,KLUPFL * MATM BASE: KAKE, VALUE: 981S99.20, ORIFT:0.09, OTHER BASES: QX41,

\begin{tabular}{|c|c|c|c|c|c|c|c|c|c|c|c|c|c|c|c|c|c|c|}
\hline $\begin{array}{l}\text { STAT } \\
\text { GSII }\end{array}$ & No & & & & & & & HT & LEY & El & $\begin{array}{l}085 \bar{V} \\
124 \mathrm{E}\end{array}$ & $\begin{array}{l}085 V \text { GRAV } \\
\text { BILLGALS }\end{array}$ & GR & GAA & $\begin{array}{r}564 \\
2.85\end{array}$ & ACOH & $\begin{array}{l}504 \\
2.67\end{array}$ & $\begin{array}{r}-5 T A T \\
-\quad \text { Bus } \\
\end{array}$ \\
\hline KAKE & BASE & * 56 & 57.53 & 133 & 54.51 & A & $*$ & 9 & 10 & $\mathrm{c}$ & 1019 & 981699.20 & B & $21 \cdot 3$ & 21.0 & 4 & 21.0 & * KAKE \\
\hline$j \times 18$ & $\angle C \mathrm{DH}$ & $\neq 56$ & 56.19 & 134 & $16-18$ & 4 & 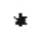 & 10 & 12 & $c$ & 1134 & 981638.92 & D & $13-1$ & 12.6 & 4 & 12.7 & $+0 \times 18$ \\
\hline $0 \times 19$ & & - 58 & 54.39 & 134 & 13.10 & 4 & $*$ & 1 & 4 & $\mathrm{C}$ & 1155 & 981691.10 & 0 & 17.0 & 16.9 & 4 & 16.9 & $-0 \times 19$ \\
\hline $7 \times 20$ & & $\$ 56$ & 3.3 .23 & 134 & 10.14 & 4 & $*$ & 3 & $b$ & c & 1209 & 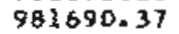 & 0 & 18.0 & & 4 & & - $\theta \times 20$ \\
\hline $0 \times 21$ & & 456 & 51.79 & 134 & 3.50 & 4 & 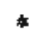 & 2 & 6 & 6 & 1224 & 981691.17 & 0 & 20.6 & 20.6 & 4 & 20.6 & $\div 0 \times 21$ \\
\hline $0 \times 22$ & & \$ 56 & 50.27 & 134 & 7.37 & A & * & l & 5 & $\mathrm{C}$ & 1242 & 981609.28 & 0 & 20.9 & 20.8 & 4 & 20.8 & $+0 \times 22$ \\
\hline $5 \times 23$ & & * 56 & 51.04 & 134 & 9.99 & a & $\Rightarrow$ & i & 5 & c & 1254 & 961690.72 & 0 & 21.3 & $21-1$ & 4 & 21.1 & - $9 \times 23$ \\
\hline $9 \times 24$ & & - 56 & 51.98 & 3.4 & 12.02 & $\mathbf{A}$ & $*$ & 1 & 5 & $c$ & 3308 & 981689.20 & 0 & 10.5 & 18.3 & 4 & 18.3 & $-0 \times 24$ \\
\hline $3 \times 25$ & & $* 58$ & 53.03 & 134 & 14.57 & A & * & 2 & 6 & $c$ & 1330 & 981693.04 & 0 & 21.0 & 20.8 & 4 & 20.6 & $* 0 \times 25$ \\
\hline $0 \times 26$ & & $\Rightarrow 56$ & 54.45 & 134 & 17.45 & A & * & 1 & 4 & $c$ & 1344 & 981692.54 & 0 & 18.3 & 18.2 & 4 & 18.2 & $-0 \times 26$ \\
\hline $2 \times 27$ & & * 58 & $54=19$ & 34 & 20.25 & A & • & 2 & 5 & c & 2400 & 961691.45 & 0 & 17,7 & [ 7,5 & 4 & 17.5 & $+0 \times 27$ \\
\hline $0 \times 28$ & & * 56 & 52.88 & 134 & 21.44 & A & * & 1 & 4 & C & 1410 & 981691.09 & 0 & 19.1 & 18.9 & 4 & 10.9 & - $0 \times 28$ \\
\hline $0 \times 29$ & & $\neq 56$ & 51.43 & 134 & 19.39 & a & $*$ & 2 & 5 & c & 1425 & 981690.30 & 0 & 20.4 & 20.2 & 4 & 20.2 & * $0 \times 29$ \\
\hline $0 \times 30$ & & $\# 56$ & 50.07 & 134 & 18.31 & A & * & 2 & 4 & $\mathrm{C}$ & 1438 & 981689.49 & D & 21.3 & 21.2 & 4 & 21.2 & - $9 \times 30$ \\
\hline $0 \times 31$ & & +56 & 48.07 & 234 & 17.06 & a & $*$ & 2 & 4 & $c$ & 1452 & $98168 B .06$ & $\mathrm{D}$ & 22.7 & 22,5 & 4 & 22.5 & - $0 \times 31$ \\
\hline $0 \times 32$ & & -50 & 47.10 & 134 & 17.79 & A & $*$ & 1 & 2 & C & 1515 & 901585.39 & $D$ & $21=1$ & 21.1 & 4 & 21,1 & $-0 \times 32$ \\
\hline $5 \times 33$ & & * 5t & 50.03 & 134 & 20.53 & a & $*$ & $\bar{z}$ & 2 & C & 1550 & 981690.94 & 0 & 22.7 & 22.6 & 4 & 22.6 & $+8 \times 33$ \\
\hline $6 \times 34$ & & $* 50$ & 51.60 & 134 & 21.87 & $A$ & $*$ & 5 & 5 & C & 1607 & 981691.53 & o & • & 21.2 & 4 & 21.2 & *0.34 \\
\hline $0 \times 35$ & & * 50 & $51-42$ & 134 & 24.03 & $\vec{A}$ & $*$ & 2 & i. & $\mathrm{c}$ & 1624 & 981693.73 & D & 23.4 & 2 & 4 & 23.4 & $=0 \times 35$ \\
\hline $0 \times 36$ & & $* 56$ & 49.76 & 134 & 25.11 & a & 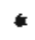 & 2 & 0 & C & 1647 & 981694.08 & D & 25.7 & 25.7 & 4 & 25.7 & - $0 \times 36$ \\
\hline $6 \times 37$ & & $=56$ & 48.53 & 134 & 24.68 & A & - & 3 & 1 & c & 1710 & 981588.82 & 0 & 22.5 & 22.5 & 4 & 22.5 & $-0 \times 37$ \\
\hline $6 \times 38$ & & * 56 & 46.91 & 134 & 23.96 & a & $*$ & g & s & $\mathrm{C}$ & 1732 & 9816 & D & 23.4 & $23 \cdot 2$ & 4 & 23 & $=9 \times 30$ \\
\hline $0 \times 59$ & & $\div 56$ & $45.5 \mathrm{H}$ & 134 & 23.32 & $\vec{a}$ & * & 3 & 0 & $c$ & 1248 & 981687.81 & 0 & 25.5 & 25.5 & 4 & 25.5 & * $0 \times 39$ \\
\hline $0 \times 46$ & & * 56 & 43.90 & $1 \pm 4$ & 24.00 & a & $\bullet$ & 7 & 4 & 0 & 1810 & 981686.39 & 0 & 26.6 & 26.5 & 4 & 26.5 & $-0 \times 40$ \\
\hline $9 \times 41$ & BASE & +50 & 43.53 & 134 & 21.47 & A & $*$ & 9 & 6 & $c$ & 1840 & $981689.8 \mathrm{~B}$ & A & 30.9 & 30.7 & 4 & 30.7 & $-0 \times 41$ \\
\hline $0 \times 42$ & & +56 & 42.25 & 134 & 22.40 & 4 & $*$ & $i$ & 3 & $c$ & 2707 & $981685+14$ & $c$ & 27.7 & 27.6 & + & 27.6 & $\because 0 \times 42$ \\
\hline $0 \times 45$ & / SUL & * 56 & 40.48 & 134 & 22.01 & $\Delta$ & 4 & 20 & 23 & $C$ & 2132 & 981679.46 & c & 26.3 & 25.5 & 4 & 25.6 & $09 \times 43$ \\
\hline $0 \times 44$ & & $* 56$ & 39.00 & 134 & 19.97 & 4 & $*$ & 1 & 5 & $\tau$ & 2153 & 981677.29 & E & 24.5 & 24.3 & 4 & 24.4 & $+0 \times 44$ \\
\hline $0 \times 41$ & DASE & $* 56$ & 43.53 & 134 & 21.47 & d & $\star$ & I & 6 & $c$ & 2228 & 981689.88 & 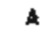 & 30.9 & 30.7 & 4 & 30.7 & - $0 \times 41$ \\
\hline KAXE & OASF & * 56 & 57.53 & 133 & 54.51 & $\mathrm{a}$ & $\bullet$ & 6 & 10 & $c$ & 3639 & 961699.20 & 8 & 21.3 & 21.0 & 4 & $21=0$ & * KAKE \\
\hline
\end{tabular}

DALA SUHEASY

NUMACA OF STATLUNS: 30

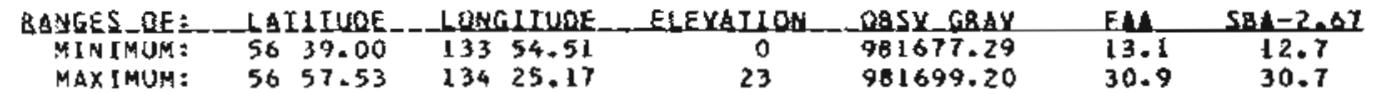




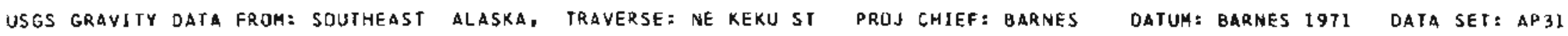
DATE: 06/29/69, MEFER: G-17, DRSERVERS : POPENOE,XLUPFL * MAIN BASE: KAKE, VALUE: 981699.20, DRIFT:0.15, OTHER BASES: ,

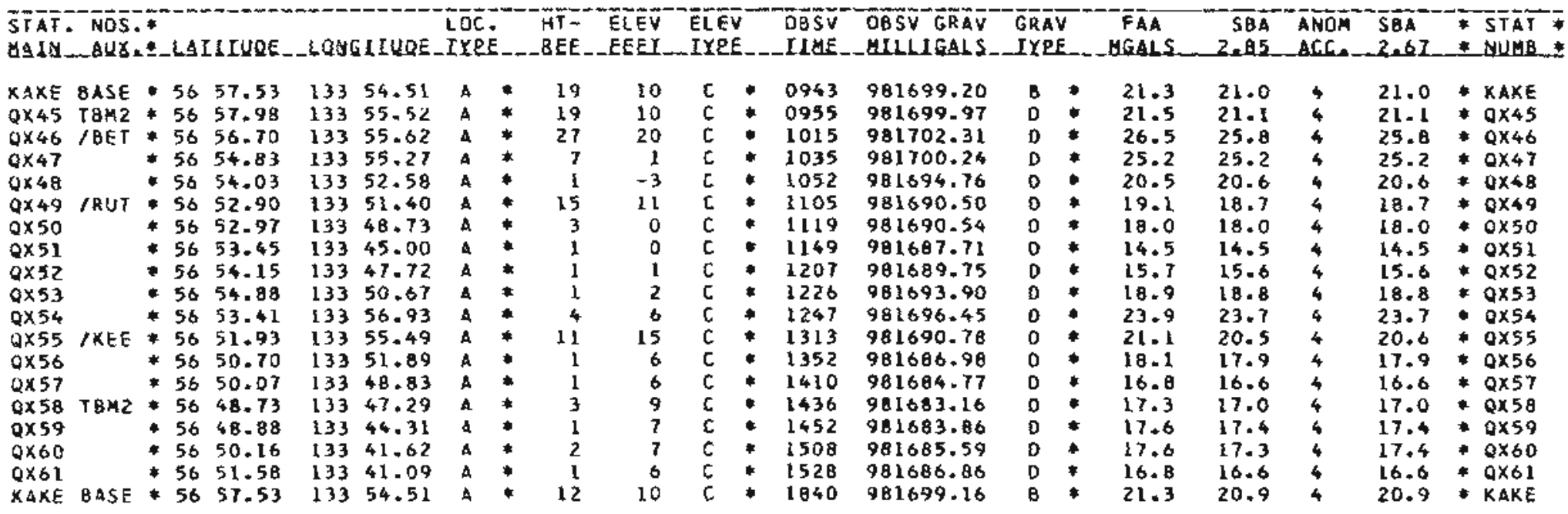

QAIA_5LULARY

NUMBER OF STATIONS: 19

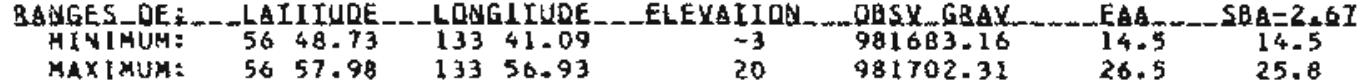




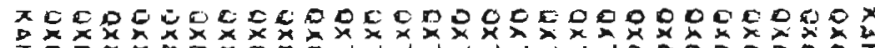

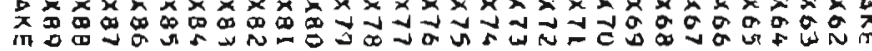

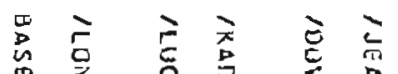

$\frac{a d}{x}$

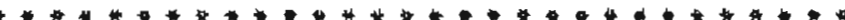

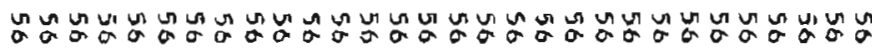

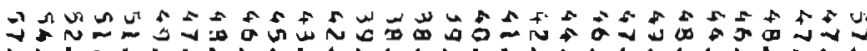

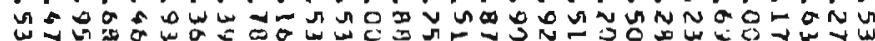

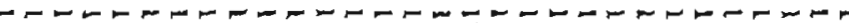

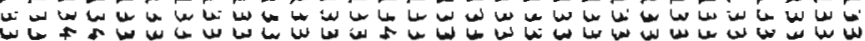
П

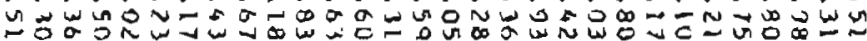

$\triangle D D D D D D D D D D D D D D D D D D D D D D D D D D D$

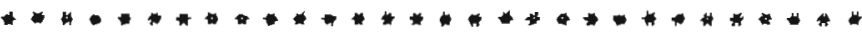

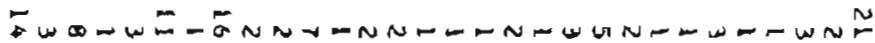

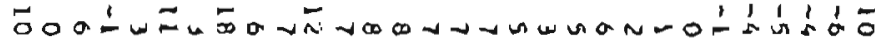

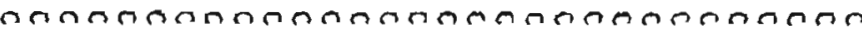

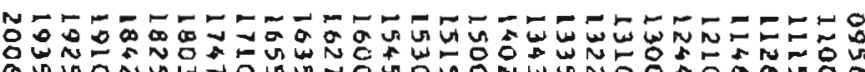

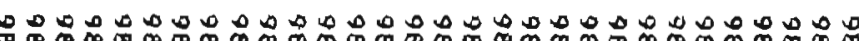

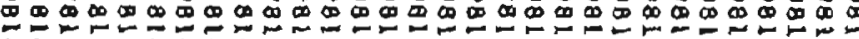

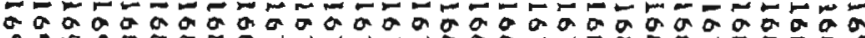
品品 -

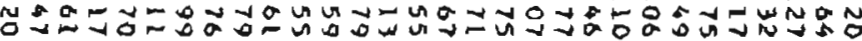
000000000000000000000000000000

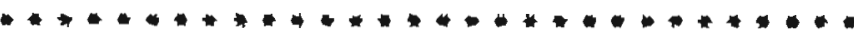

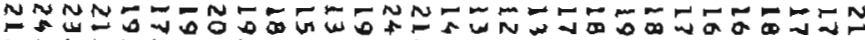

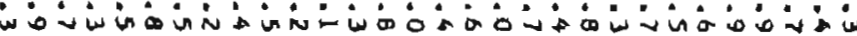

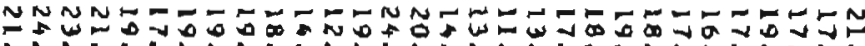
o 5. $+\ldots+\ldots+\ldots$

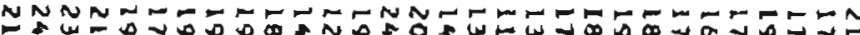

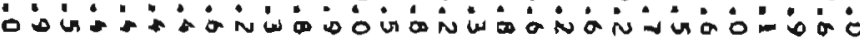

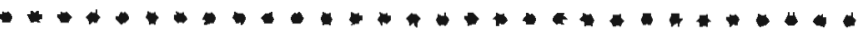

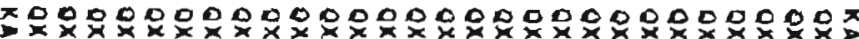

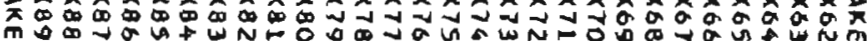




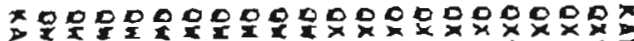

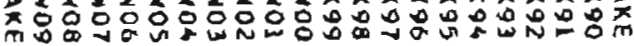

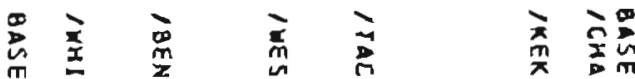

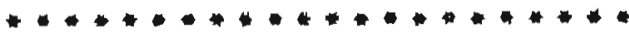

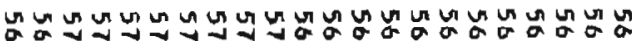
$m m$ M้

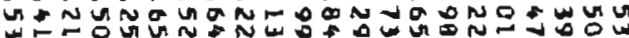

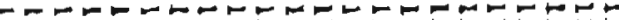

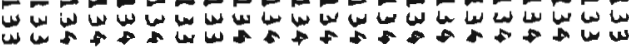

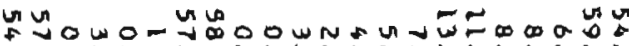

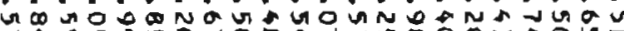

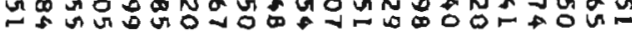
$\Delta x \pi r \pi-D+x D D D D D D D D D$

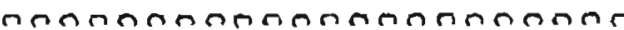

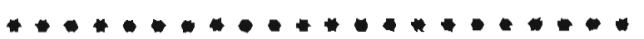

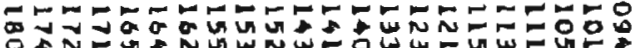

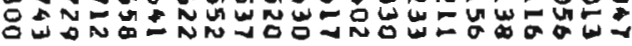

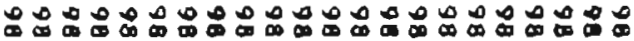

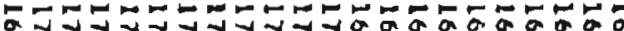

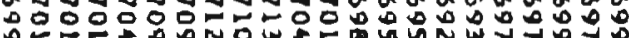

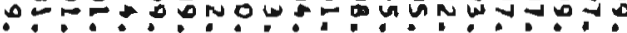

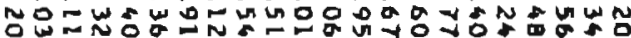
1000000000000000000000

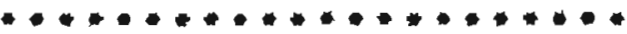

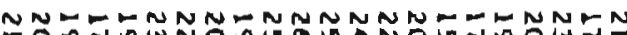
ำ:

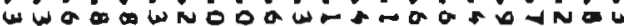

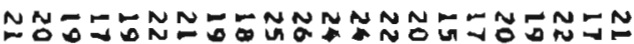
TO:

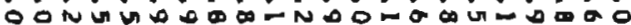

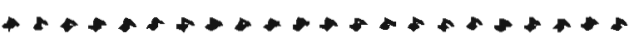

NOFニ

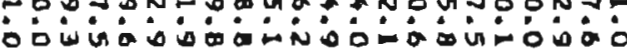

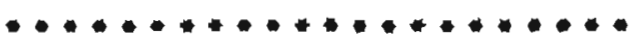

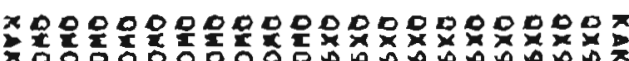

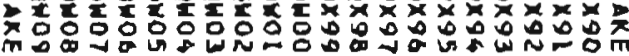




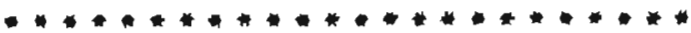

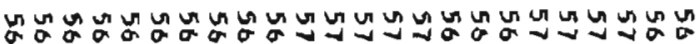

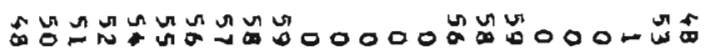

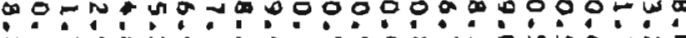

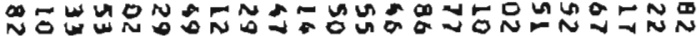

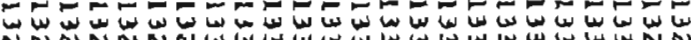

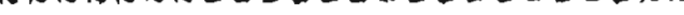

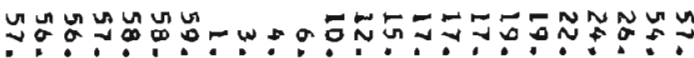

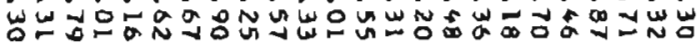

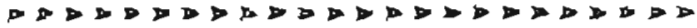

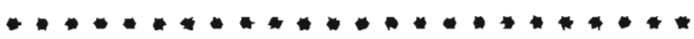

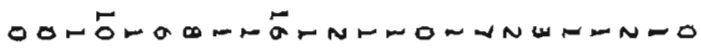

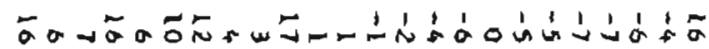

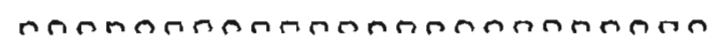

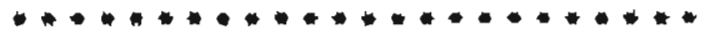

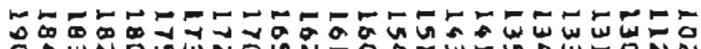

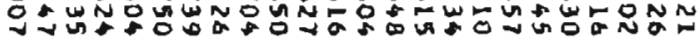

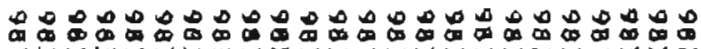

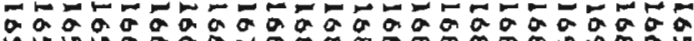

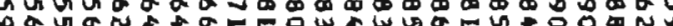

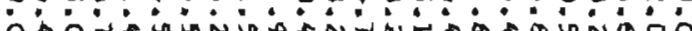

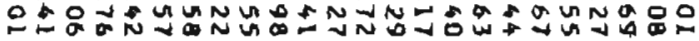

$-0000000000000000000000$

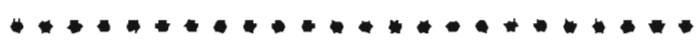

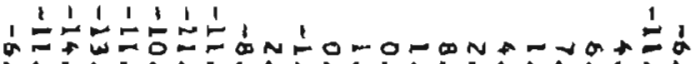

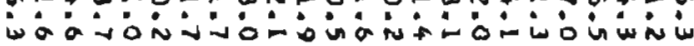
$12 \frac{1}{2} \frac{1}{2} \frac{1}{2}$ $\pm 6$

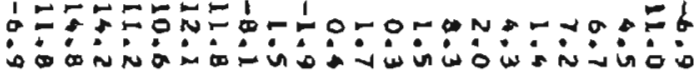
$n+n+n+\ldots+n+n+n+n$

$1=\frac{1}{1} \frac{1}{2} \frac{1}{8} \frac{1}{2}$ 1

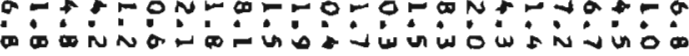
-

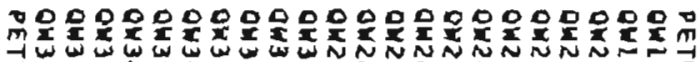

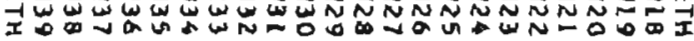


USGS GRAYETY DATA FQDH: SUUTHEAST ALASKA, TRAVERE = AGASIL PEN PROJ CHIEF = BARMES

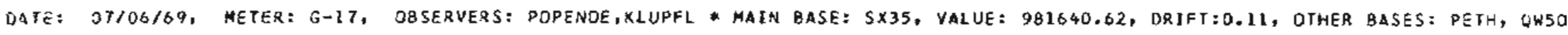

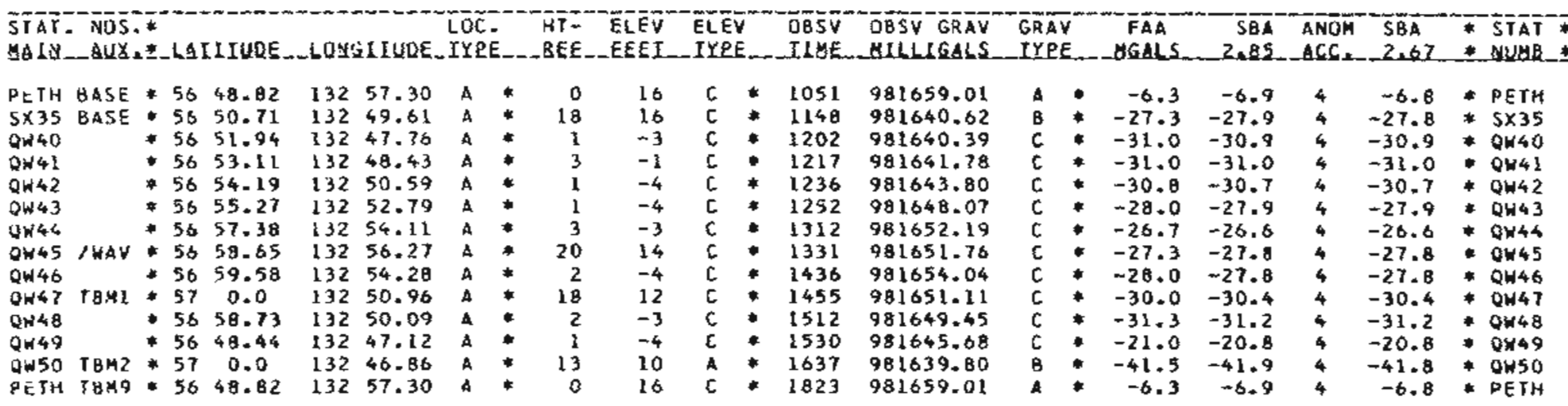

\section{DAIA SUUGASY}

HUMBER OF STATIDNS: 14

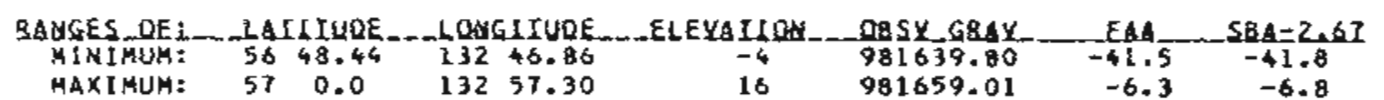




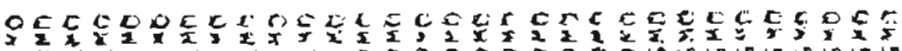

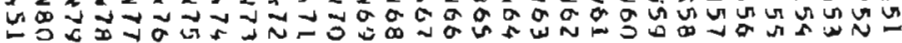
兽号

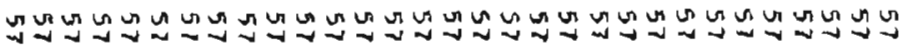
б

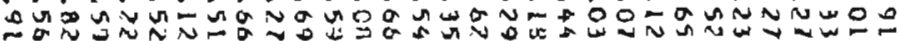

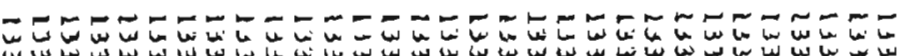
బ

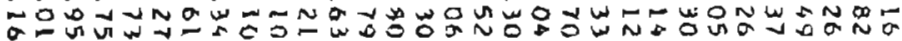

$\triangle D D D D D D D D D D D B D D D O D D D D D D D D D D$

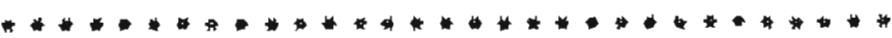

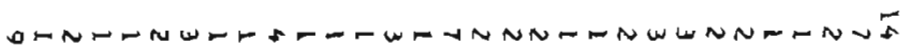

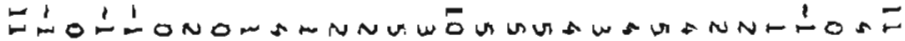

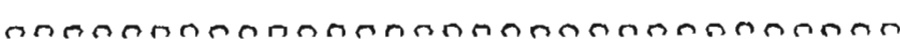

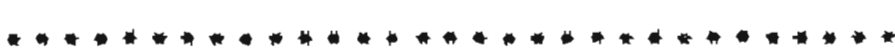

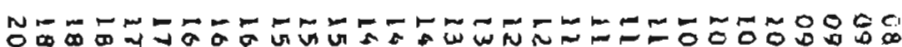

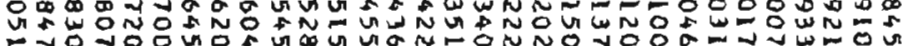

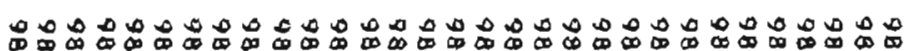

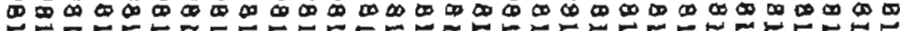

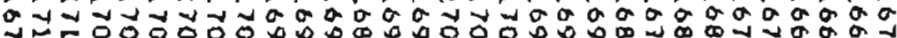
ज品

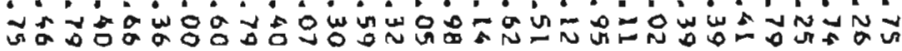
000000000000000000000000000000

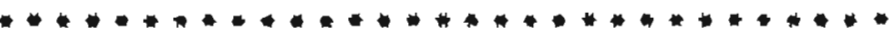

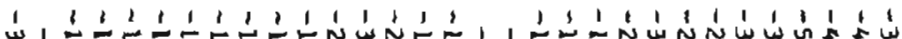

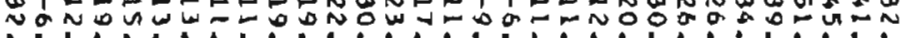

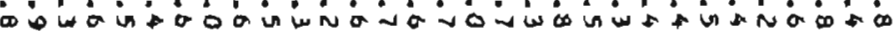

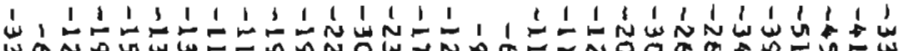

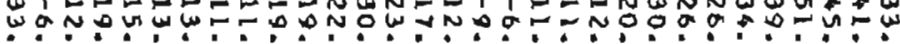

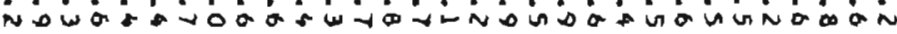

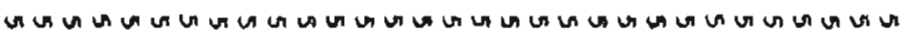

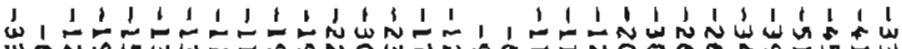

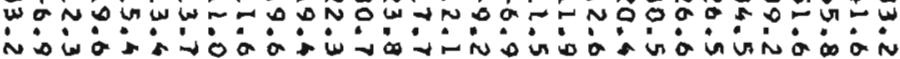

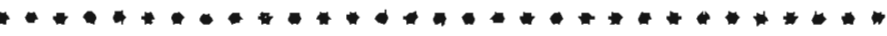

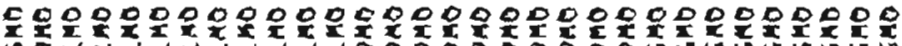

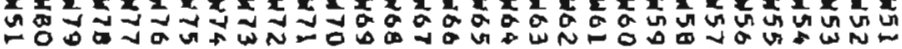




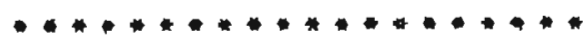

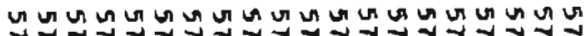

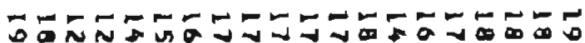

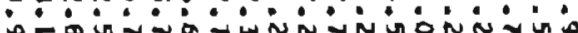

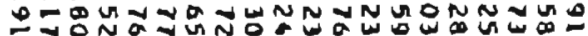

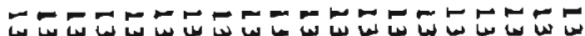

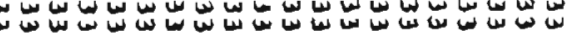

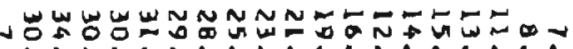

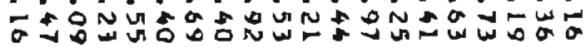

ODDDDDDDDDDDDDD

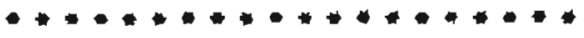

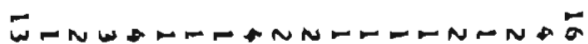

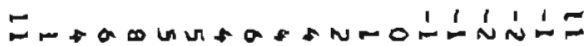

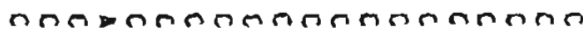

$\ldots \ldots \ldots \ldots+\ldots \ldots+\ldots \ldots$

วัดง

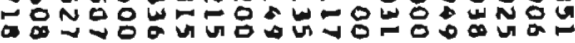

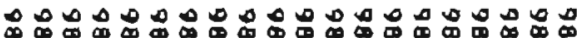

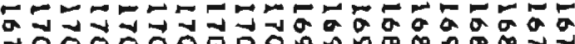

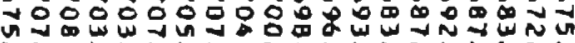

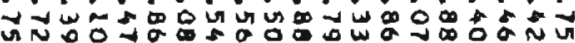

क ก n

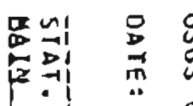

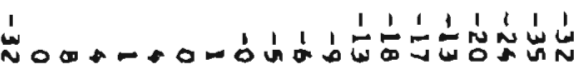

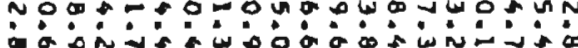

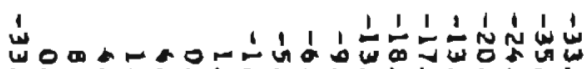
U:

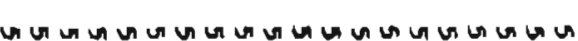

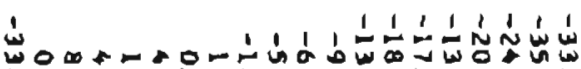

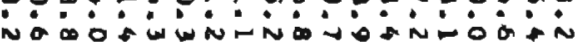




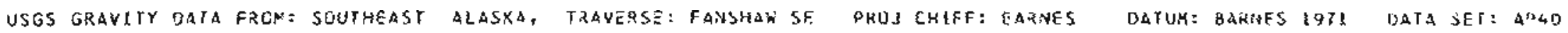

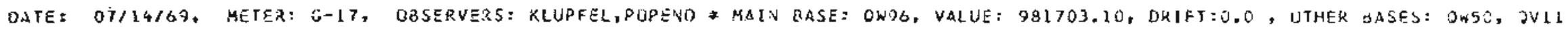

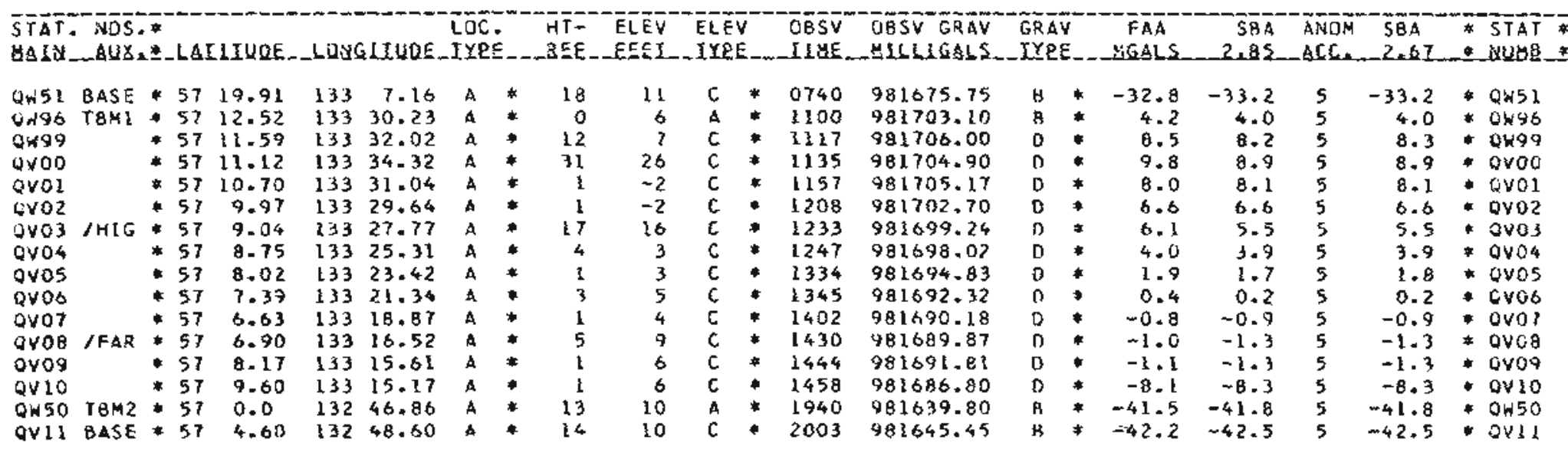

DATA FOR T/L 469 SUGGEST BASE UNCERTALNTIES OP METER PRORLEMS

\section{DAIA_SUGHABY}

NUHEER OF STATIONS: L6

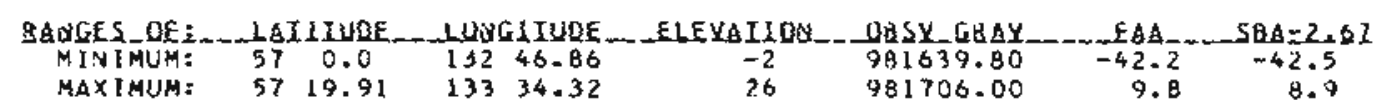


USOS GKAVITY DATA FUUH: SOUTHEAST ALASKA. TRAVERSE: IHOMAS-FAR PRUJ THIEF: BARMES DATUME BARNES 1971 OATA SET: APG1

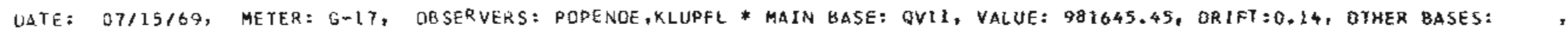

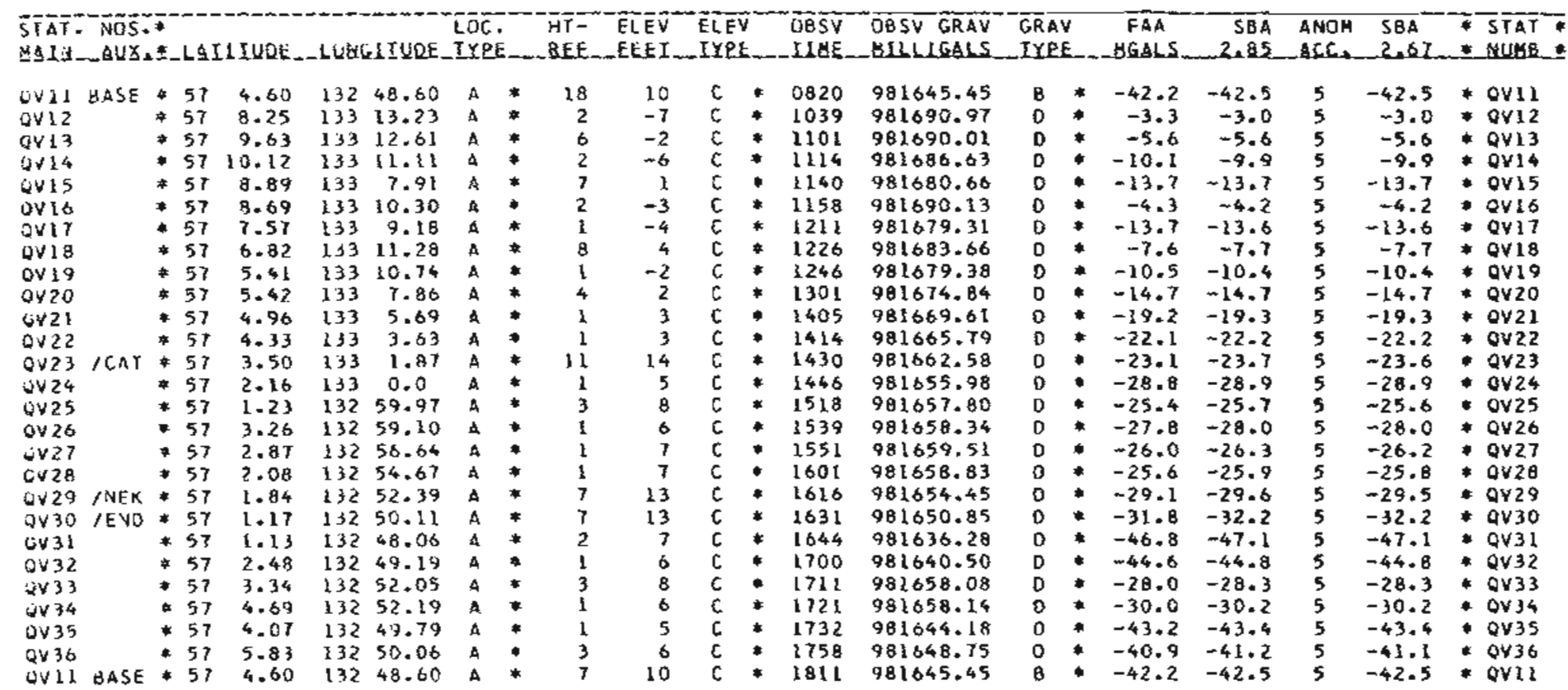

DATA_SHEGABY

NUHHER UF STATIJNS: 27

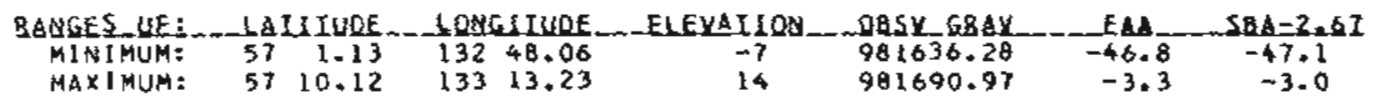


DAIE: 27/17/69, MEFLR: G-17, UHSERVERS: KLUPFEL,PUPENO \# MAIH BASE: OVI1, VALUE: 981645.45, ORIFT:0.06, OTHER BASES: SX35,

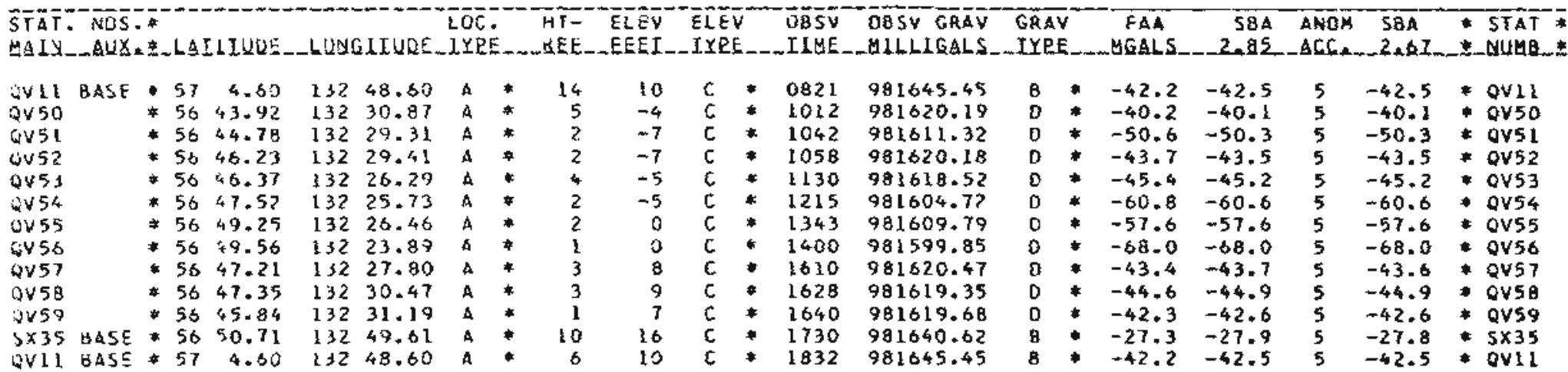

DALASUUYABY

FUMBER IF STAISTYS: IN

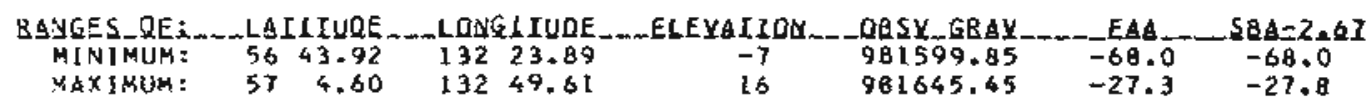

USGS GRAVITY DATA FROM: SOUTHEAST ALASKh, TRAVERSE: FARRAGUT R PROJ CHIEFE BAANES OATUH: BARNES L9TI DATA SET: APG3 DATE: $27 / 1 B / 69$, METER: G-1T, UBSERVERS: POPENOE

- HAIN BASE: qV3T, VALUE: 981689.45, ORTFT:0.03, OTHER BASES: QV37.

\begin{tabular}{|c|c|c|c|c|c|c|c|c|c|c|c|c|c|c|c|c|c|c|c|c|c|c|}
\hline $\begin{array}{l}\text { IAJ } \\
\text { AI }\end{array}$ & & & & & & & & $\begin{array}{l}T- \\
\text { FE }\end{array}$ & $F$ & & $\bar{s} \bar{v}$ & S5V & & & & 2 & - & $\overline{584}$ & $\begin{array}{l}\text { STAT } \\
\text { nuHa }\end{array}$ & $\begin{array}{l}5 \\
\\
4\end{array}$ & & \\
\hline $\begin{array}{l}0 \times 37 \\
0 \times 38 \\
0 \times 39 \\
0 \times 40 \\
9 \times 41 \\
0 \times 42 \\
0 \times 43 \\
0 \times 45 \\
0 \times 37 \\
0437\end{array}$ & A5E & $\begin{array}{l}57 \\
* 57 \\
* 57 \\
* 57 \\
* 57 \\
* 57 \\
* 57 \\
* 57 \\
* 57 \\
* 57\end{array}$ & $\begin{array}{r}8.74 \\
10.41 \\
10.73 \\
10.65 \\
11.50 \\
12.02 \\
13.62 \\
15.15 \\
8.74 \\
0.74\end{array}$ & $\begin{array}{l}132 \\
132 \\
132 \\
132 \\
132 \\
133 \\
133\end{array}$ & $\begin{array}{l}1.78 \\
7.39 \\
0.33 \\
0.33\end{array}$ & $\begin{array}{l}A \\
B \\
B \\
A \\
A \\
A \\
A \\
A \\
A \\
A\end{array}$ & 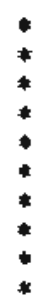 & $\begin{array}{l}2 \\
0 \\
0 \\
0 \\
0 \\
0 \\
0 \\
3 \\
11 \\
10\end{array}$ & $\begin{array}{r}373 \\
550 \\
794 \\
445 \\
6 \\
6\end{array}$ & $\begin{array}{l}c \\
v \\
v \\
v \\
v \\
v \\
v \\
v \\
z \\
c\end{array}$ & $\begin{array}{r}2044 \\
+\quad 2055 \\
+\quad 2104 \\
+\quad 2142 \\
+\quad 2308 \\
\quad 3340\end{array}$ & 981689.45 & $\begin{array}{l}B \\
C \\
c \\
c \\
c \\
c \\
C \\
C \\
b \\
B\end{array}$ & 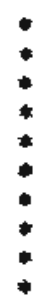 & $\begin{array}{r}-4.2 \\
-19.2 \\
-21.5 \\
-23.6 \\
-30.4 \\
-40.1 \\
-42.1 \\
-31.5 \\
-4.2 \\
-4.2\end{array}$ & $\begin{array}{r}-4.5 \\
-20.2 \\
-24.7 \\
-27.4 \\
-44.0 \\
-60.1 \\
-70.7 \\
-47.8 \\
-4.5 \\
-4.5\end{array}$ & $\begin{array}{l}5 \\
6 \\
6 \\
6 \\
6 \\
6 \\
6 \\
6 \\
6 \\
5\end{array}$ & $\begin{array}{r}-68.9 \\
-46.7 \\
-4.4 \\
-4.4\end{array}$ & 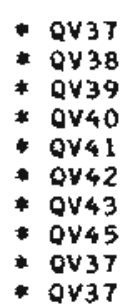 & $\begin{array}{r}6 \\
20 \\
90 \\
120 \\
320 \\
570 \\
940 \\
446\end{array}$ & $\begin{array}{l}y \\
x \\
k \\
k \\
x\end{array}$ & $\begin{array}{r}-4.4 \\
-20.7 \\
-24.8 \\
-26.3 \\
-46.3 \\
-57.7 \\
-59.6 \\
-46.7\end{array}$ \\
\hline
\end{tabular}

altimetry traVERSE Closed on lake farragut, check photogrametay eleVation

DAIA_5ututeY

SuMaER jF SIATLINS: 10

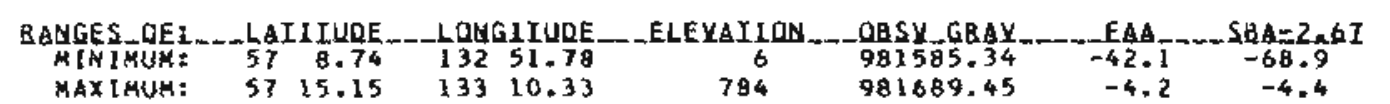




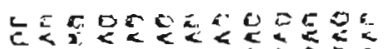

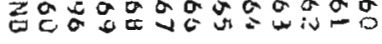

果牙

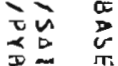

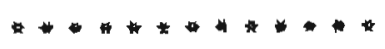

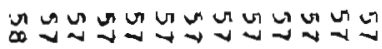

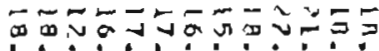

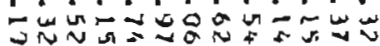

ヒーッーールのこーテこここ

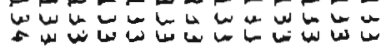

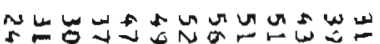

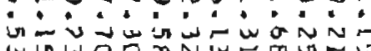

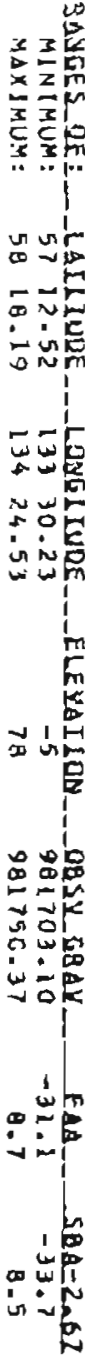

$D D D D D D D D$

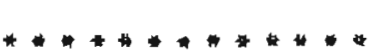

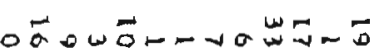

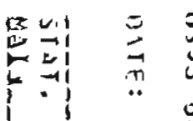

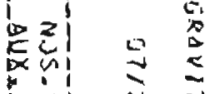

$\because \frac{1}{1}$ 永

Dabnommanan

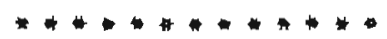

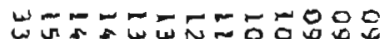

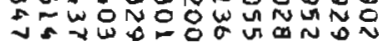

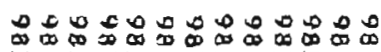

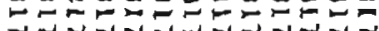

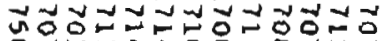
군?

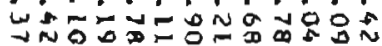

$-\infty 00000000000$

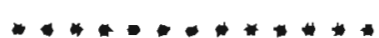

$\stackrel{3}{-}$

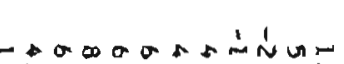
on

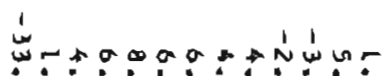

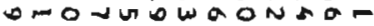

- unu un un vin un us

出-

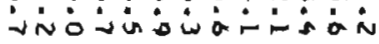

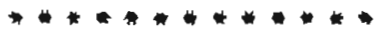

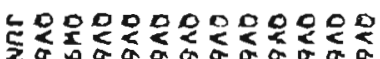




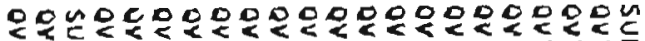

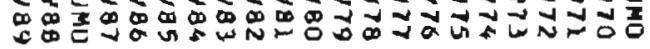
$\stackrel{\text { s }}{\mathrm{i}}$

营宫

$\frac{\pi}{0}$ 罦

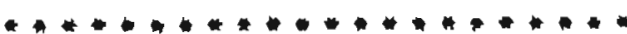

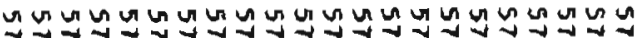

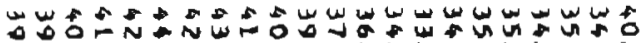

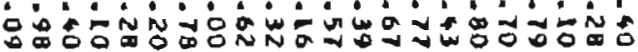

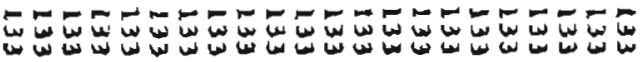

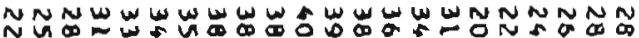

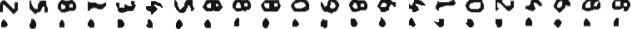

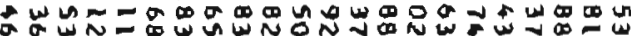

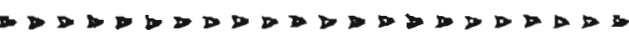

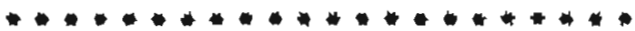

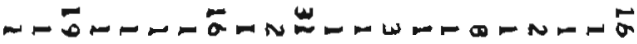

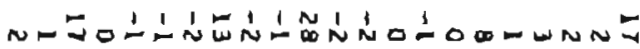

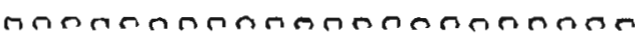

$\ldots \ldots \ldots \ldots \ldots \ldots \ldots \ldots+\ldots \ldots \ldots$

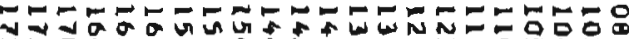

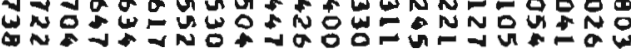

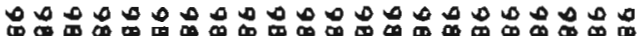

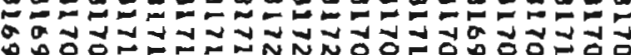

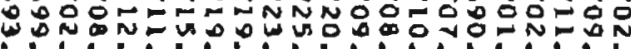
ogn

$00 \$ 000000000000000000$

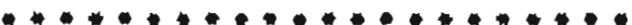

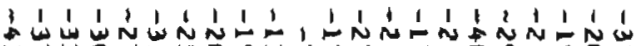
幽出

1

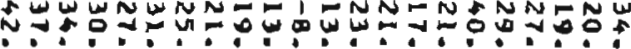
OU U U

$n+a n+h+h+h+n+h+h+n$

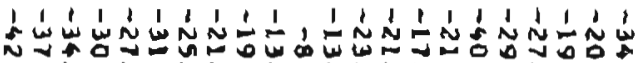

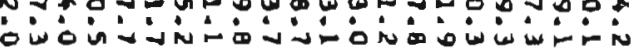

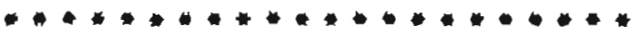

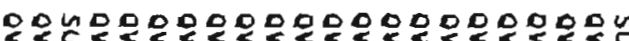

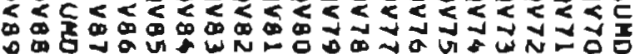


ง

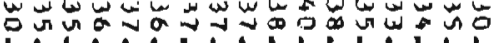

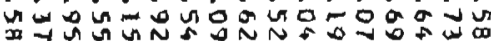

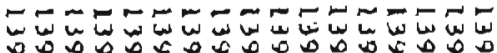

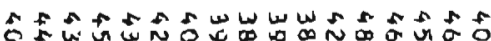

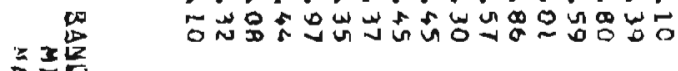

蓝 $D D D D D D D D D D D D$

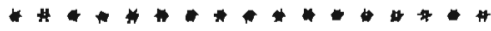

O-FWRN-M-INNNT-M

$\sin$

○岁点

o in

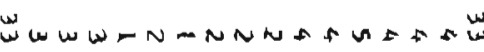

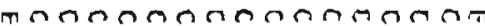

w它

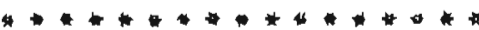

का एक

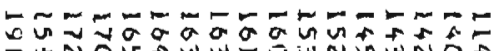

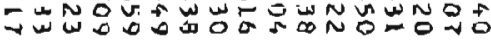

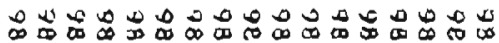
$\approx \sim 20$

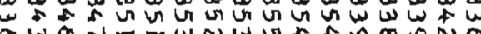

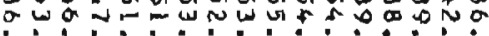

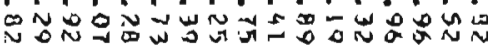

ynanmananghan

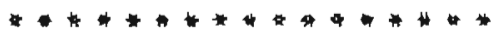

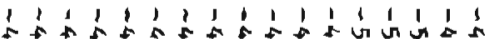

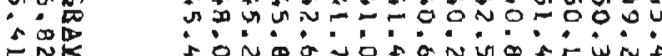

年

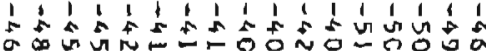

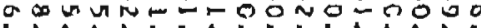

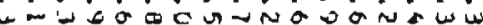
ahahahahananhath

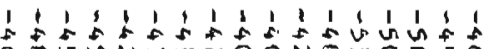

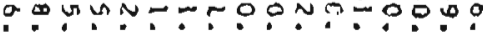
in w $\begin{gathered}0 \\ 0\end{gathered}$

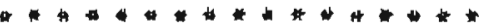

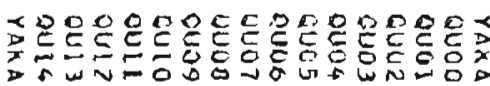


USGS GRAVITY DAIA FROM: SOUTHEAST ALASXA, TRAYERSE: RUSSEL RRM PROJ CHIEF: BAANES DATUM: BARNES L9TL DATA SET: ALOL

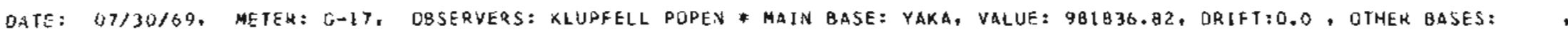

\begin{tabular}{|c|c|c|c|c|c|c|c|c|c|c|c|c|c|c|c|c|c|c|}
\hline 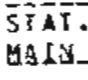 & $\begin{array}{r}-40 \\
-40\end{array}$ & & 10 & & & & & & EY & 5 & $\begin{array}{l}\text { DESV } \\
\text { LUE }\end{array}$ & $\begin{array}{l}\text { OASV GRAV } \\
\text { UILLIGALS }\end{array}$ & & AGAL & $\begin{array}{r}584 \\
2.20\end{array}$ & & $\begin{array}{r}5 B A \\
-2 A 6\end{array}$ & $\begin{array}{r}\text { STAT } \\
\text { * MUSB }\end{array}$ \\
\hline YAKA & BASE & +59 & $30.5 \mathrm{a}$ & 137 & 40.10 & A & * & 0 & 33 & $\mathrm{E}$ & 0922 & 981836.82 & A & -45.4 & -46.3 & 4 & -46.5 & * \\
\hline Qง 15 & & 59 & & 139 & 28.09 & $A$ & 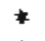 & 1 & -2 & $\bar{c}$ & 1140 & 981852.78 & $\bar{E}$ & -70.9 & -70.8 & 4 & -70.8 & - Qu15 \\
\hline 9416 & & * 59 & & 139 & $2 T .04$ & $A$ & * & 4 & 2 & c & 1156 & 981852.86 & $F$ & -69.0 & -69 & 7 & -69.1 & - Oulá \\
\hline 0017 & & * 59 & 57.13 & 139 & 25.70 & A & • & 2 & 0 & $c$ & 1209 & 981855.91 & E & -64.2 & -64.2 & 4 & -64.2 & - oult \\
\hline Quis & & * 59 & 56.12 & 139 & 23.90 & A & $*$ & 1 & 3 & $c$ & 1323 & 981863.32 & $E$ & -55.2 & -55.3 & 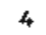 & -55.3 & - Qula \\
\hline oula & & * 59 & & 139 & 23.03 & A & * & 2 & 4 & $c$ & 1335 & $9 B 1 B 63.78$ & $E$ & -53.6 & -53. & 4 & -53.7 & - Qu19 \\
\hline Qu20 & & $* 59$ & $54-07$ & 139 & 22.30 & 4 & $*$ & 2 & 5 & c & 1347 & 981882.20 & $\vec{E}$ & -53.5 & -53.6 & 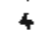 & -53.7 & - 0020 \\
\hline Qu21 & & - 50 & 53 & 139 & 21.16 & A & * & 2 & 5 & $\varepsilon$ & 1355 & $9818 \times 1.62$ & $E$ & -52.9 & -53.0 & 4 & -53.0 & * Qu2l \\
\hline 0422 & & * 59 & 52. & 139 & 14.97 & A & $*$ & 2 & 5 & c & 1406 & 981859.65 & E & -53.9 & -54.0 & 4 & -54.1 & - Qu22 \\
\hline ou 23 & & * 57 & 51.33 & 139 & 18.96 & A & $*$ & I & 5 & $c$ & 1417 & $981865.7 \mathrm{t}$ & $\bar{E}$ & -48.4 & -46.5 & 4 & -46.8 & - 0u23 \\
\hline QU24 & & $* 59$ & 50. & 139 & 19.85 & A & * & 1 & 5 & c & $142 a$ & 9816 & E & -43.7 & -43.8 & 4 & -43.8 & - Qu24 \\
\hline 0025 & & * 59 & 49.20 & 139 & 20.85 & A & $*$ & 2 & $b$ & c & 1447 & 981869.04 & $E$ & -40.2 & -40.3 & 4 & -40.4 & - Qu2s \\
\hline 0426 & & * 59 & 47.77 & 139 & 21.33 & A & * & 2 & 6 & $c$ & 1500 & 981867.25 & E & -40.1 & -40.3 & 4 & -40.3 & - 9426 \\
\hline euz7 & & - 59 & 46. & 139 & 21.47 & $A$ & * & 2 & 0 & c & 1510 & 901 & $\mathrm{E}$ & -38.4 & -38.6 & 4 & -3 & \# Qu2 \\
\hline QU 28 & & * 59 & 45.32 & 139 & $21 \cdot 53$ & A & + & I & 5 & $\mathrm{c}$ & 1522 & 981869.49 & 6 & -34.7 & -34.9 & 4 & -34.9 & - Qu2a \\
\hline OU29 & $Y K 26$ & - 59 & 44.33 & 139 & 21.00 & A & $\bullet$ & 1 & 5 & $c$ & 1532 & 981669.30 & E & -33.6 & -33.8 & 4 & -3 & * 0429 \\
\hline 0030 & & * 59 & 43.06 & 139 & 20.40 & A & - & 3 & 7 & c & 1545 & 9818 & $E$ & -3 & -33 & 4 & & - Qu30 \\
\hline ou 31 & & $* 59$ & 41.60 & 139 & 19.30 & A & * & 1 & 5 & $c$ & 1600 & 901068.52 & E & -30.0 & -30.9 & 4 & $-31 * 0$ & * Qusl \\
\hline Lu $3 ?$ & & - 59 & 40.09 & 134 & 19.03 & $A$ & - & 1 & 5 & c & 1615 & 961871.09 & $E$ & -26.3 & -26.4 & 4 & -26.4 & * 0032 \\
\hline Q013 & & $* 59$ & 38.91 & 119 & 17.74 & $A$ & $*$ & 3 & 7 & c & 1625 & 981868.21 & $\mathbf{E}$ & -27.4 & -27.6 & 4 & $-27,7$ & * 9033 \\
\hline 0436 & & $=59$ & 37.54 & 139 & 17.61 & A & * & 2 & 5 & C & 1640 & 981876.91 & E & -17.1 & -17.2 & 4 & -17.3 & * QU34 \\
\hline Qu 35 & & - 57 & 36.52 & 139 & $28 \cdot 20$ & 4 & * & 2 & 5 & $c$ & 1658 & 981878.26 & E & -14.5 & -14.6 & 4 & -1 & - Qu35 \\
\hline Qu 36 & YK27 & * 59 & 35.84 & 139 & 20.20 & A & * & 2 & 4 & $c$ & $170 \%$ & 981877.17 & $E$ & -14.7 & -14 & 4 & $-14,9$ & - Qu 36 \\
\hline 0437 & & 459 & 34.59 & 139 & 19.49 & A & $*$ & 1 & 3 & c & 1720 & $9 B 1872.15$ & E & -19.2 & -18.2 & 4 & -18.3 & * 6037 \\
\hline $0 \cup 3 a$ & & +59 & 13.15 & 139 & 17.52 & A & * & 2 & 3 & $\mathrm{c}$ & 1737 & $98 \angle 675.2 \mathrm{~B}$ & E & -13.9 & -14.0 & 4 & -14.0 & + 0038 \\
\hline 0u39 & & * 59 & 34.31 & 139 & 15.57 & A & * & 2 & 3 & c & 1747 & 981877.00 & E & -13.0 & -13.0 & 4 & -13.1 & * 60159 \\
\hline Q04i & & - 59 & 35.41 & 139 & 15.22 & 4 & $*$ & 4 & 5 & c & - 1757 & 981874,85 & E & $*-16.3$ & -16.5 & 4 & -16.5 & * QU40 \\
\hline
\end{tabular}

CLOSURE NOT POSSIBLE ON NIGHT OF 7/30/69,NEXT DAY DRIFT $<0.05$ MGAL

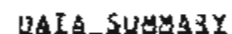

MUEBER JF STATLUNS = 27

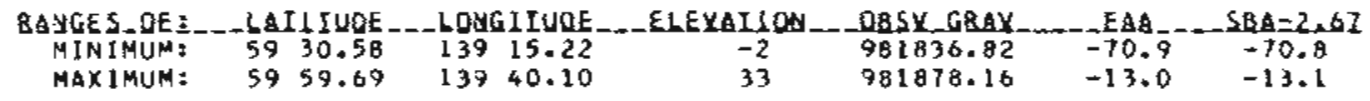




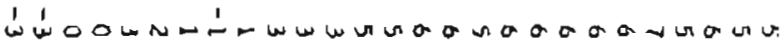

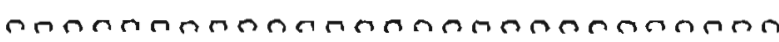

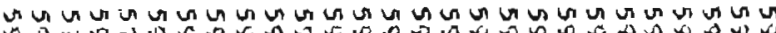

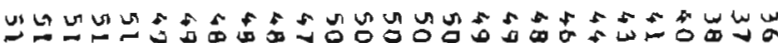

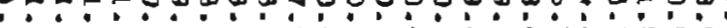

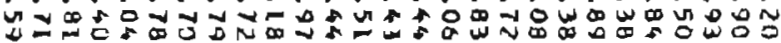

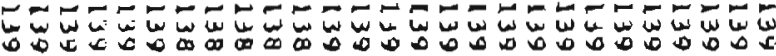

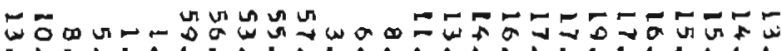

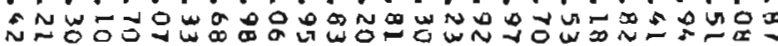
$\triangle D D D D D D D D D D D D D D D D D D D$

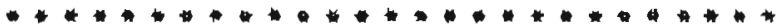

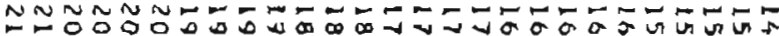

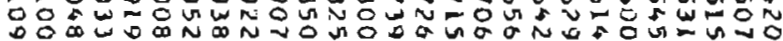

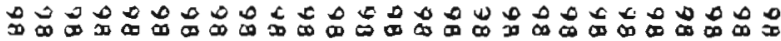

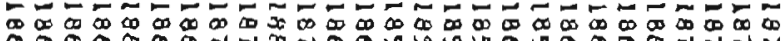

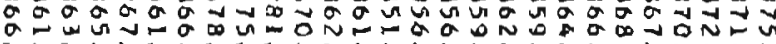

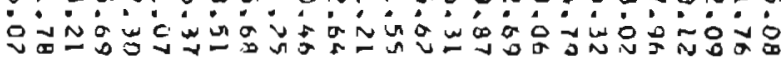

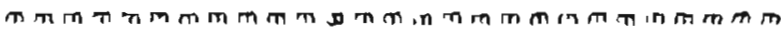

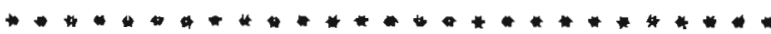

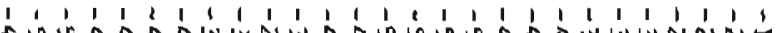

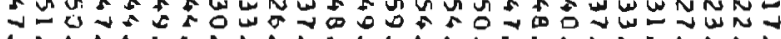

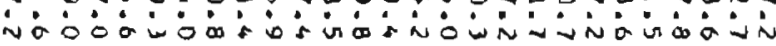

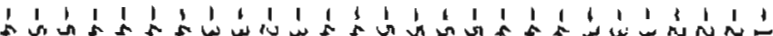
ح. .

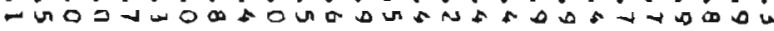

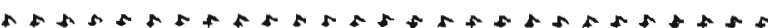

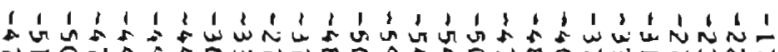

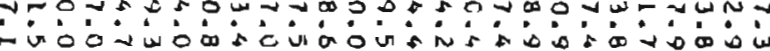

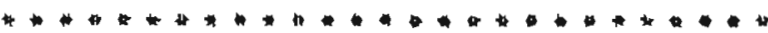

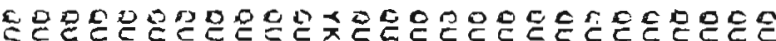
a 


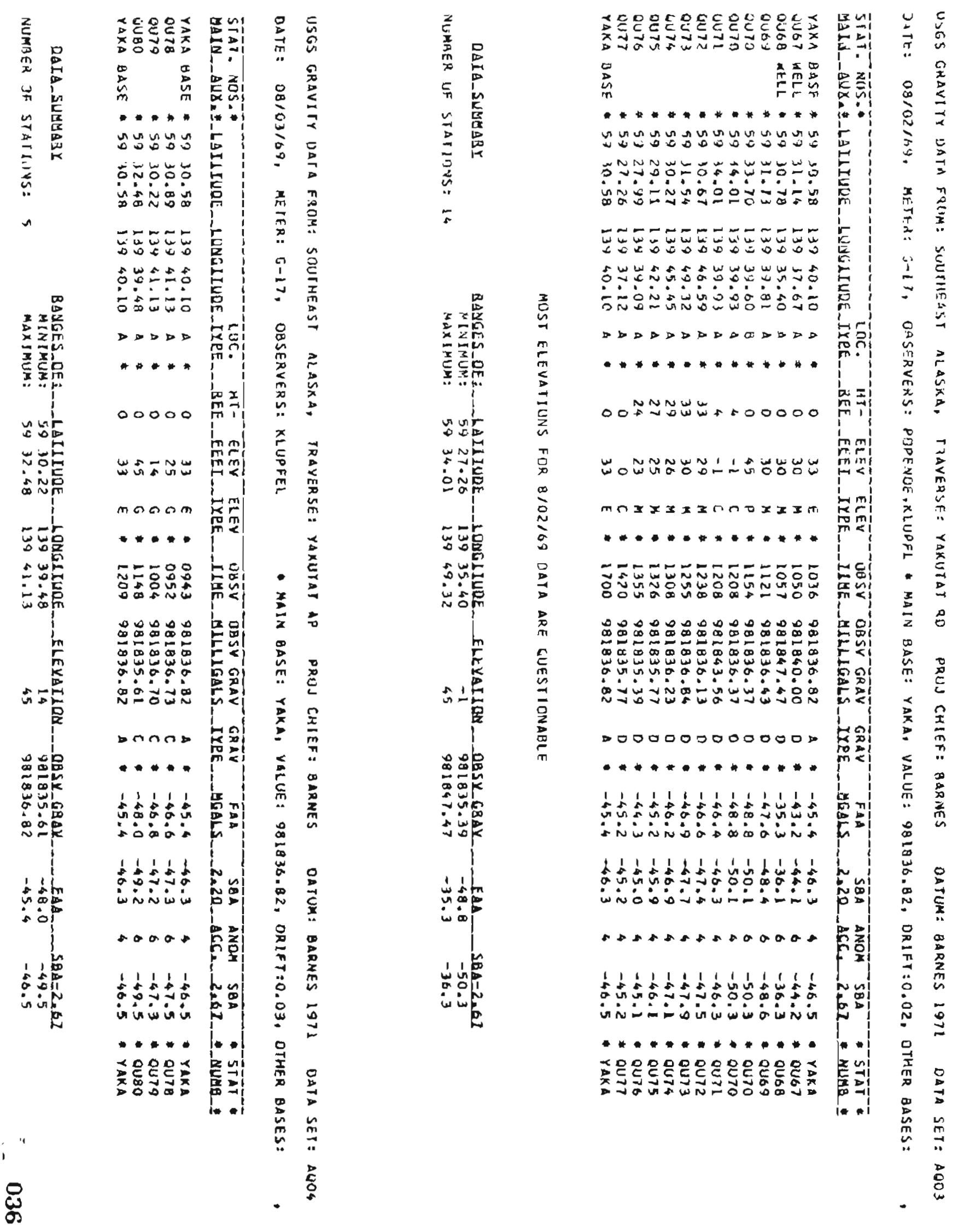




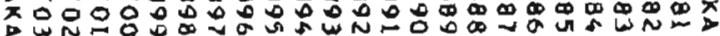
$\stackrel{\infty}{\infty}$

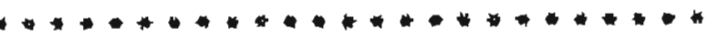

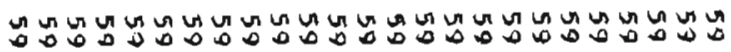

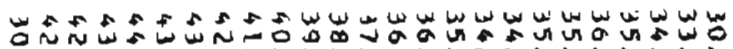

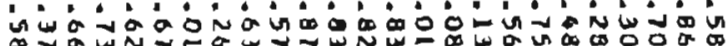

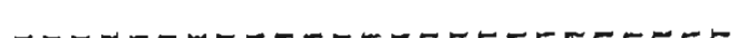

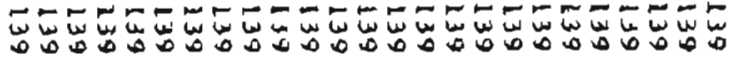
合岕山嵌

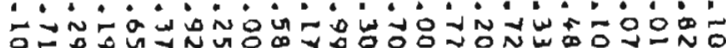

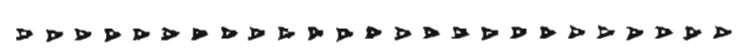

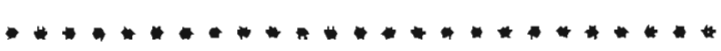

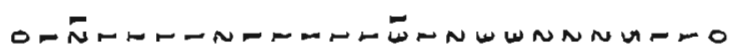

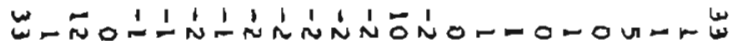

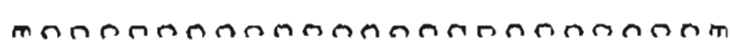

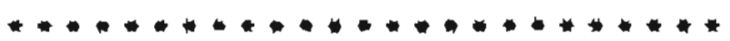

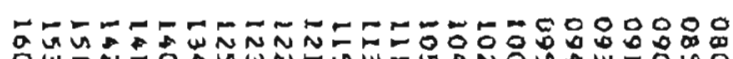

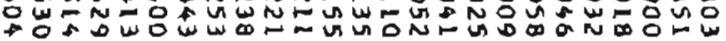

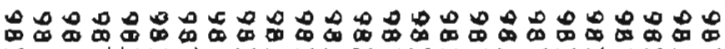

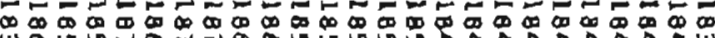

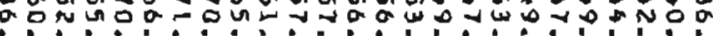

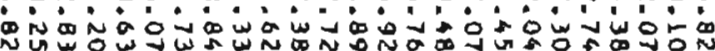

NU

-00000000000000000000000

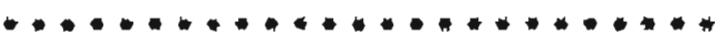

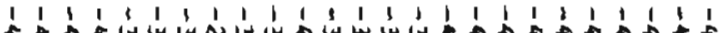

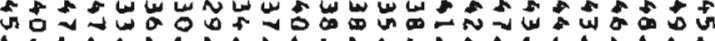

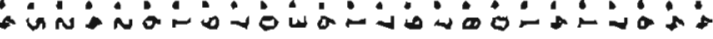

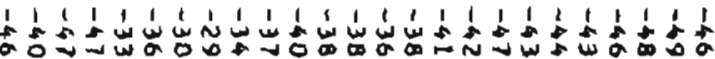

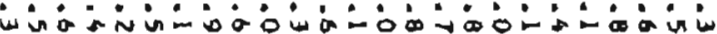

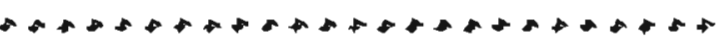

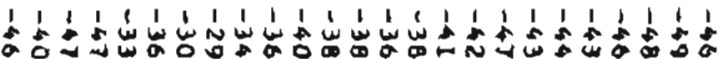
in

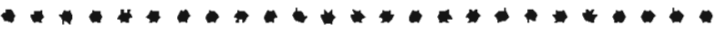

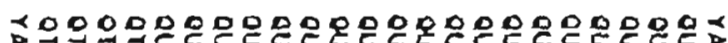

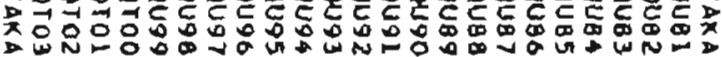




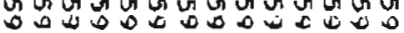

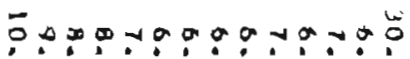

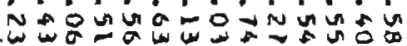

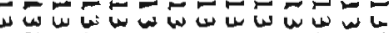

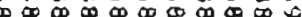

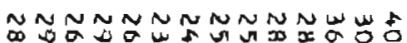

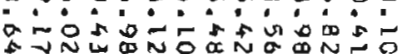

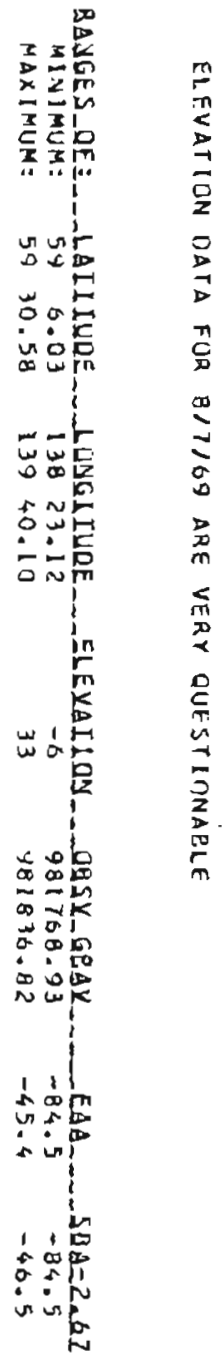

$D D D D D D D D D D$

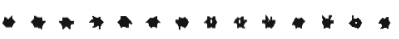

000000 T00ก范出

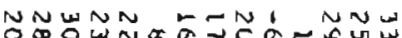

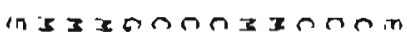

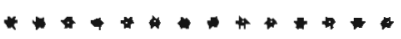

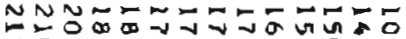

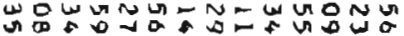

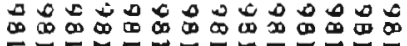

テปัง

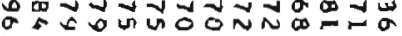

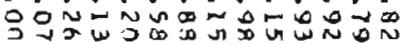

$=0000000000005$

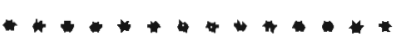

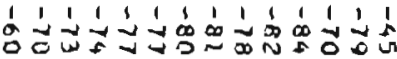

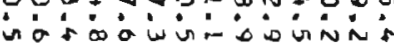

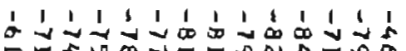
Disiso in

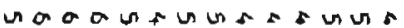

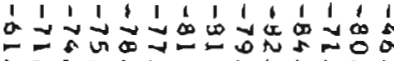

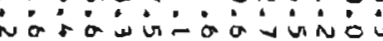

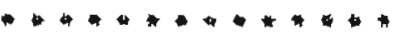
00000000000001

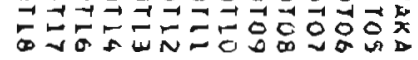




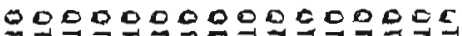

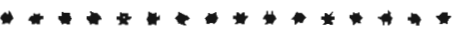

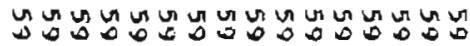

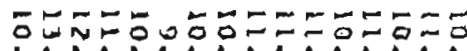

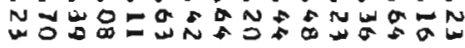

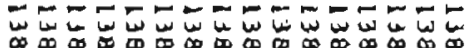

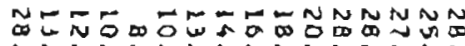

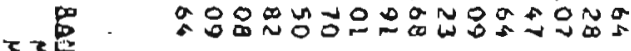
$\square \square D D D D D D D D D$

$\cdots+\ldots+\cdots+\ldots$

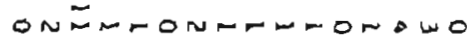

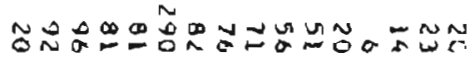

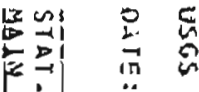

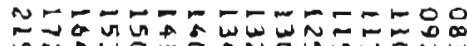

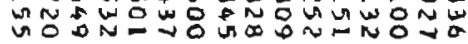

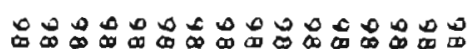

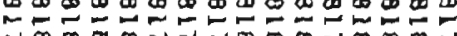
○ N

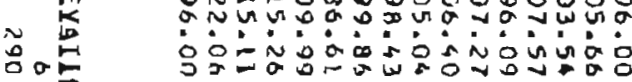
1000000000000000

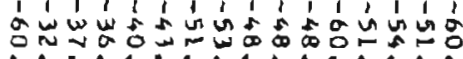

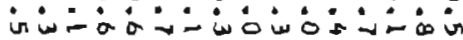

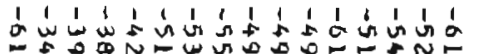
年

d.

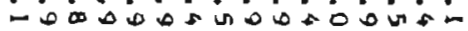

nagagaogadounaus

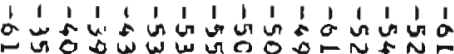

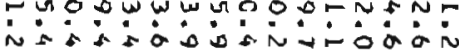

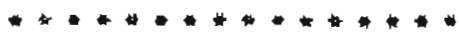

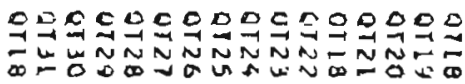

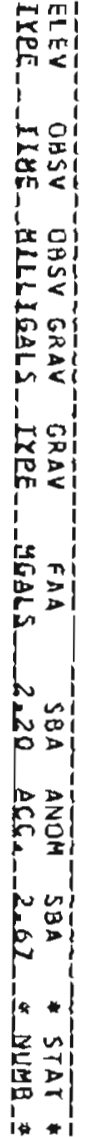



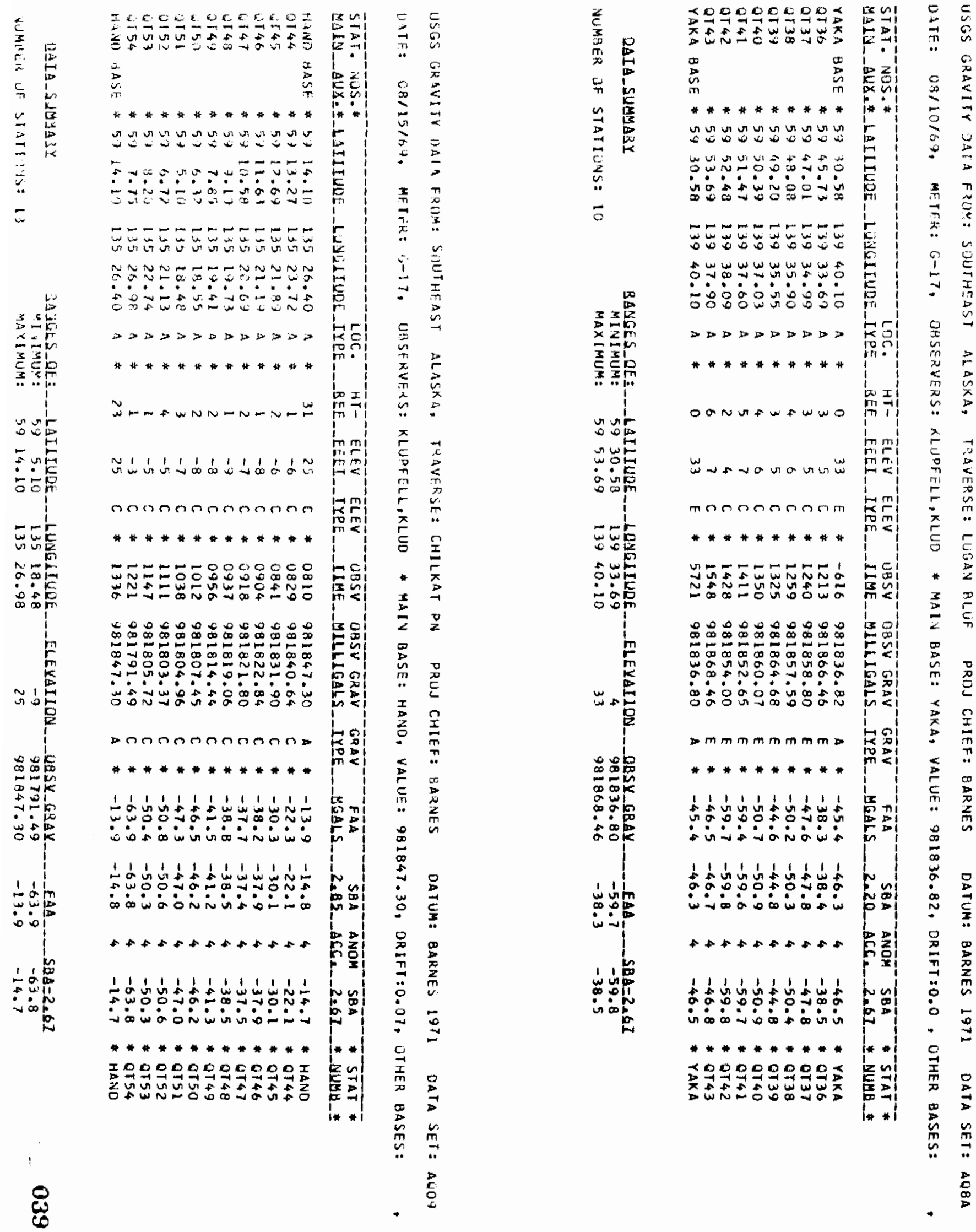
USGS GRAVITY OATA FROM: SOUTHEAST ALASKA, TRAVERSE: CHILKAT RD PRUJ CHIEF: BARNES DATUM: GARNES 1971 OATA SET: AOLO DATE: OG/16/69, METER: L-17, OBSERVERS: KLUPFELL,KLUO PAIN BASE: HAMO, VALUE= 9B1047.30, DRTFT:-.09, OTHER BASES: HANP,

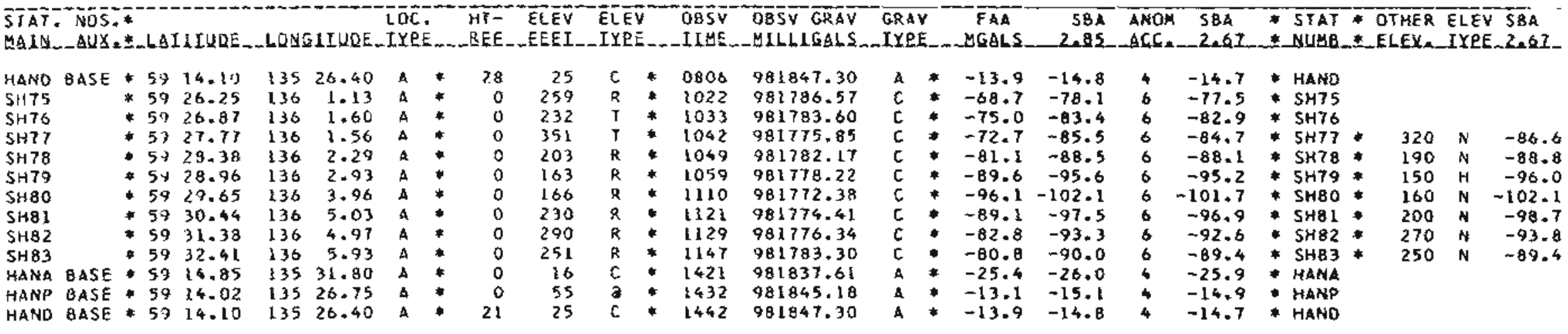

AL I IMETRY FOR O/16/69 GRAVITY DATA OBTAINED ON $8 / 29 / 69$

DAI a SUHYA8Y

NUMBER OFF STAT JNS: 13

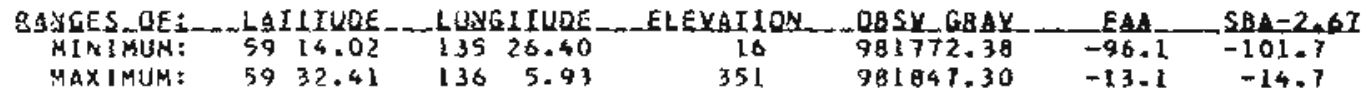




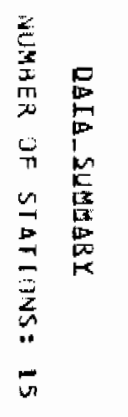

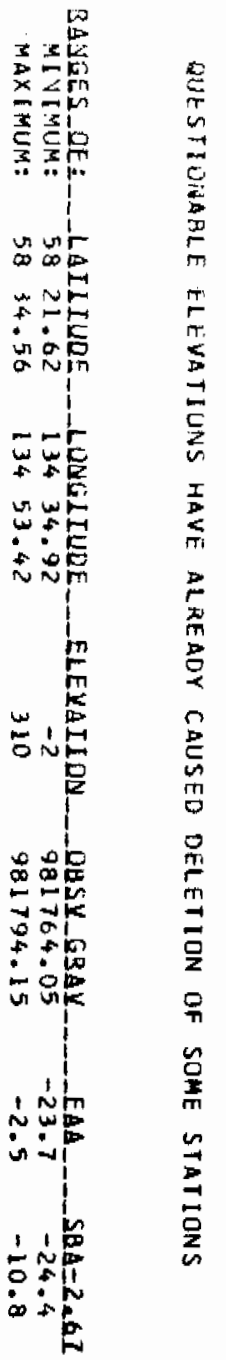

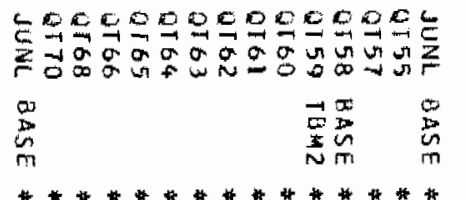

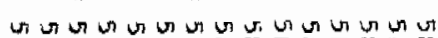

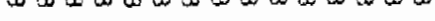

UN

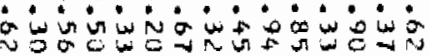

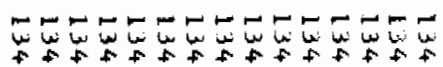

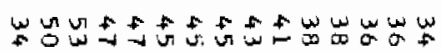

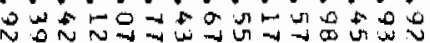

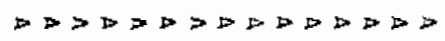

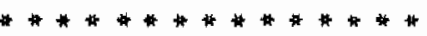

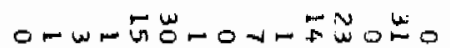

$\sum_{i=1} \frac{9}{m}$

in

乩忌

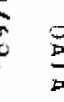

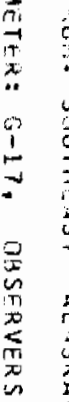

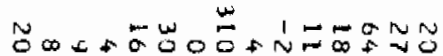

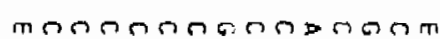

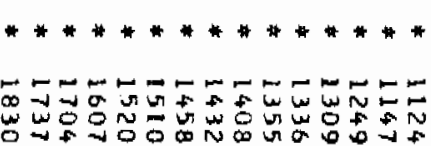

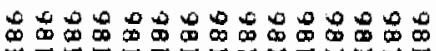

大 $\checkmark$ U.

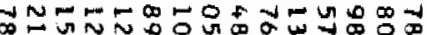

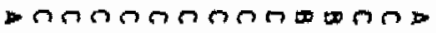

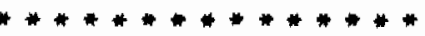

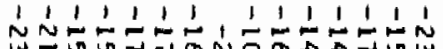

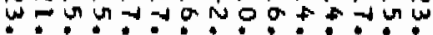

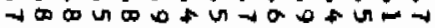

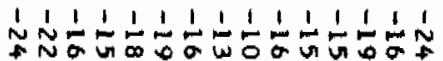

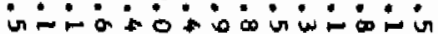

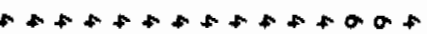

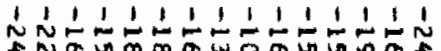

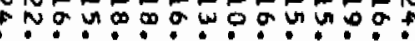

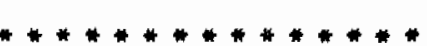

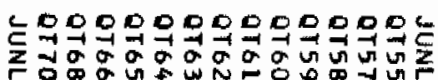


WSGS GRAVITY UAFA FRDA: SMUFAEAST ALASRA, TRAVERSE JUNEAU RO?

PAOJ CHIEF: BARNES

OATUM: BARYES 1971

DATA SET: AGIL

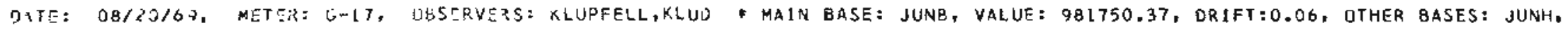

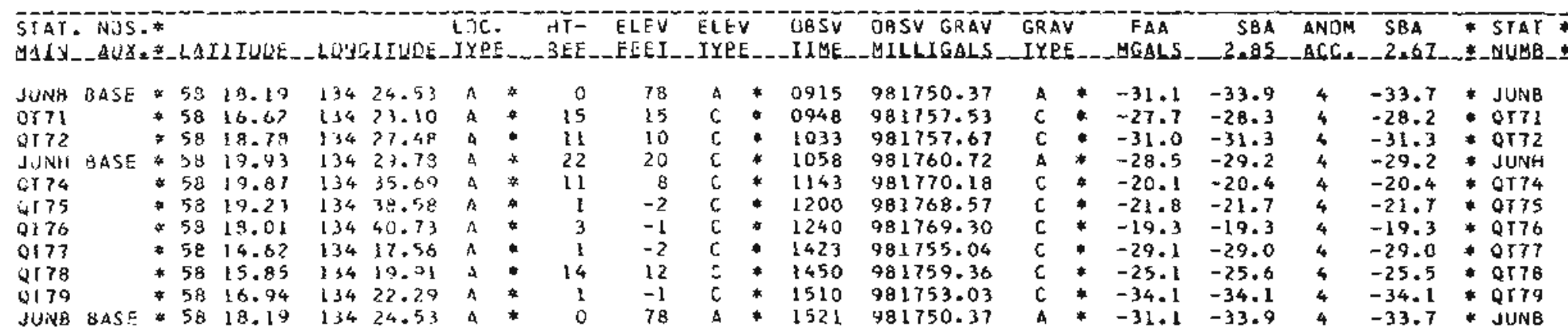

WUESTIJYADLE CLEVATIUNS HaVE aLREADY CAUSED DELETION OF SOME SFATIONS

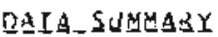

WMAER OF SIATEJW= I

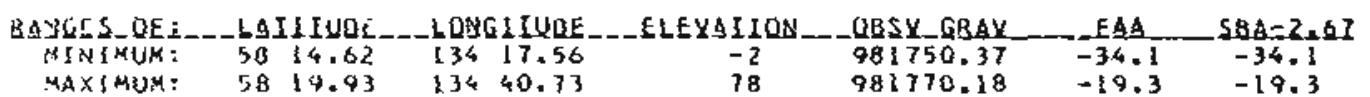


USOS GRAYITY DASA FMON: SOUTHEAST ALASKA, TQAYERSE: WHITE PASS PROJ CHIEF: BARNES DATUM: BARNES 1971 DATA SET: AQ45

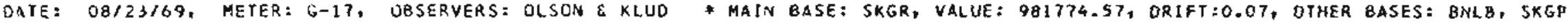

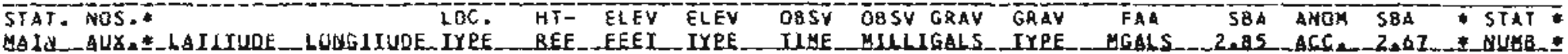

\begin{tabular}{|c|c|c|c|c|c|c|c|c|c|c|c|c|c|c|c|c|c|c|c|c|}
\hline SKGR & BASE & +50 & 27.26 & 135 & 19.98 & $A$ & * & 0 & 22 & a & * & 559 & $9 B 1774.57$ & A & * & $-104 \cdot 3$ & -105.1 & 4 & -105.0 & - $5 K G R$ \\
\hline $5 \times 6 P$ & BASE & $* 50$ & 27.40 & 135 & 19.80 & $A$ & 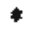 & 0 & 22 & & & 610 & 981773.74 & 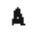 & & $-105 \cdot 3$ & -106.1 & 4 & -106.0 & - SkGP \\
\hline Wp 1 & 6-A & * 55 & 27.38 & 135 & 18.59 & 4 & * & 0 & 23 & & & 616 & 982772.98 & $c$ & & -105.9 & $=106.8$ & 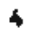 & -108.7 & - HP \\
\hline SKGR & BASE & +59 & 27.26 & 135 & 18.98 & A & - & 0 & 22 & 8 & 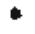 & 659 & 981774.57 & $A$ & - & -104.3 & -105.1 & 4 & -105.0 & $5 * 6 R$ \\
\hline & $B A 31$ & * 50 & 28.11 & 135 & 17.64 & A & $\star$ & 0 & 68 & & * & 741 & 981766.35 & D & * & -109.3 & $-111, B$ & 4 & -111.6 & * WP 2 \\
\hline WP 3 & 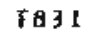 & - 59 & 28.77 & 235 & 16.82 & A & $\star$ & -6 & 131 & 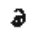 & + & 750 & 981759.00 & D & * & -111.6 & -116.4 & 4 & -116.1 & $*$ WP \\
\hline We 4 & TMP 4 & * 59 & 29.33 & 135 & 16.18 & A & * & v & 170 & & 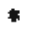 & 803 & 901754.94 & 0 & * & -112.7 & -118.9 & 4 & $-11 \mathrm{a} \cdot 5$ & * NP 4 \\
\hline & 8031 & -59 & 10.08 & 135 & 14.59 & A & - & 0 & 321 & A & * & 820 & 981750.46 & D & - & -104.0 & -115.7 & $T$ & -115.0 & * WP 5 \\
\hline$H F$ & $3-0$ & - 5 & 30.59 & 135 & 14.94 & A & - & 0 & 640 & $A$ & - & B29 & 981735.27 & D & - & -89.9 & -113.2 & $\checkmark$ & -111.7 & WP \\
\hline HP & $8-6$ & +50 & 31.33 & 135 & 13.92 & A & * & 0 & 736 & A & * & 835 & 981725.21 & 0 & 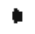 & -91.9 & -118.7 & 4 & -117.0 & - MP \\
\hline$H P B$ & & 50 & 32,17 & 135 & 12.36 & A & * & 0 & 995 & a & * & 858 & 981706.82 & D & * & -97.0 & -123.3 & 4 & -121.0 & *WP \\
\hline w9 9 & $8-F$ & * & 32.82 & 135 & 11.50 & $B$ & * & 0 & 1181 & 4 & * & 944 & 981697.57 & D & $*$ & -79.7 & -122.7 & 4 & -119.9 & * WP \\
\hline WPLO & BF 3 I & $\# 5$ & 33.67 & 135 & 10.99 & A & * & 0 & 1377 & A & - & 955 & 981689.79 & $b$ & 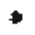 & -70.1 & -120.3 & 4 & -117.1 & - HPLO \\
\hline WP 11 & [G31] & $* 50$ & 3.3 .44 & 135 & 8.43 & $A$ & - & -6 & 3140 & $a$ & * & 1024 & 981669.66 & D & - & -55.8 & -119.2 & 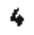 & -115.2 & WP 11 \\
\hline प्रP12 & $\mathrm{T}-\mathrm{AH}$ & +50 & 33.17 & 135 & $7.2 \mathrm{~T}$ & A & * & 0 & 1074 & $a$ & - & 1031 & 981660.19 & 0 & * & -52.4 & -120.0 & 4 & -116.3 & * $\mathrm{MP} 12$ \\
\hline WPL3 & $T-6 J$ & $* 5$ & 34.27 & 135 & 8.98 & A & * & -2 & 2258 & $a$ & * & 1044 & 981642.88 & 0 & * & -35.0 & -117.2 & 4 & -112.0 & HPLI \\
\hline WP14 & & $*$ & 35.32 & 135 & 9.01 & A & * & 0 & 2475 & $a$ & * & 1058 & 3.53 & D & + & -25.4 & -11 & 4 & 48 & 914 \\
\hline Whis & $4 d 31$ & $* 5$ & 30.10 & 135 & 0.56 & $A$ & - & $\sigma$ & 2671 & A & - & 1104 & 981623.14 & 0 & * & -18.4 & -115.6 & 4 & -10 & - WP15 \\
\hline MP 16 & $\operatorname{\theta CC} A$ & +5 & 37.49 & 135 & 8.22 & A & - & 0 & 2915 & A & - & 1117 & 981611.94 & $D$ & - & -8.5 & $-114,6$ & 4 & -107.9 & HP 10 \\
\hline WP17 & $8-14$ & $\bullet$ & 37.98 & 135 & 8.00 & $\mathrm{x}$ & - & 0 & 2890 & A & • & 1200 & 981612.43 & D & * & -12.0 & -117.2 & 9 & -110.5 & $* W P 17$ \\
\hline WP18 & $8-\times 4$ & +5 & 38.77 & 135 & 7.23 & L & * & 0 & 2913 & A & - & 1206 & 981610.39 & 0 & * & -11.9 & -117.9 & 4 & $-1 ! 1-2$ & - MPlB \\
\hline$M P 19$ & $B-J 4$ & $* 5$ & 39.55 & 135 & 6.50 & L & * & 0 & 2939 & $A$ & * & 1209 & 901609.86 & D & - & -11.0 & -118.0 & 4 & $-12 l \cdot 2$ & HP 19 \\
\hline$w \rho 20$ & $t-B 4$ & $* 5$ & 40.03 & 135 & 5.70 & $\mathbf{L}$ & * & 0 & 2933 & A & * & 1226 & 981609.55 & 0 & - & -12.5 & -119.3 & & -112.5 & - MP20 \\
\hline 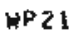 & $0-64$ & - & 40.75 & 135 & 4.65 & $k$ & - & 0 & 2931 & $A$ & - & 1303 & 981612.07 & 0 & - & $-11 \cdot 1$ & -117.8 & 4 & -111.1 & $\$ 021$ \\
\hline WP 22 & $B-C 4$ & 45 & 43.40 & 135 & 1.47 & $\mathrm{x}$ & - & 0 & 2771 & A & - & 1358 & 981622.98 & 0 & * & -18.7 & -119.6 & 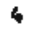 & -113.2 & w922 \\
\hline BNLB & BASE & $* 5$ & 50.80 & 134 & 59.70 & $\mathrm{k}$ & - & 0 & 2155 & $a$ & * & 1451 & 981655.99 & B & * & -53.3 & -131.6 & 4 & -126.6 & BNLE \\
\hline WP 23 & $B-\sqrt{3}$ & $* 5$ & 50.83 & 134 & 59.49 & k & $*$ & 0 & 2155 & $A$ & - & 1503 & 981655.37 & 0 & * & -56.0 & -132.4 & 4 & -127.5 & * WP23 \\
\hline WP24 & $d-w 3$ & $* 5$ & 47.20 & 135 & 0.75 & $\mathbf{L}$ & $\star$ & 0 & 2641 & A & \# & 1545 & 981636.20 & D & * & -22.7 & -118.9 & & $-112 * 0$ & * HP24 \\
\hline$M 25$ & $8-23$ & -5 & 44.73 & I 34 & 58.25 & $k$ & 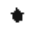 & 0 & 2833 & $A$ & * & 1609 & 981623.13 & D & * & -14.5 & -117.6 & 4 & -111.1 & HP25 \\
\hline$M P 26$ & B-va & -5 & 43.04 & 135 & 2.75 & $x$ & - & o & 2765 & A & - & 1622 & 981622.12 & D & - & -10.7 & -120.3 & & $-114,0$ & - WP26 \\
\hline SKGP & BASE & $\neq 5$ & 27.40 & 135 & 18.80 & A & \# & a & 22 & 2 & $*$ & 1050 & 981773.74 & $\downarrow$ & * & $-105+3$ & $-100,1$ & & -106.0 & $5 \times 6 P$ \\
\hline & BASE & 5 & 27.26 & 135 & 28.98 & A & $*$ & 0 & 22 & a & * & 1857 & 981774.57 & A & $*$ & -104.3 & -105.1 & 4 & -105.0 & * SKGR \\
\hline
\end{tabular}

DAI SUEA

NUMAER DF STATJUNS: 32

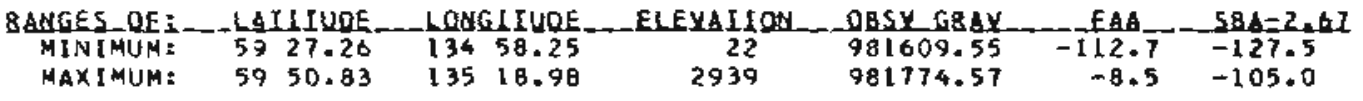


USGS GRAVITY DATA FRUM: SOUTHEAST ALASKA, TBAVERSE: AUKC BAY N PROJ CHIEF: BARNES DATUH: BARNES 19TK DATA SET: AP53

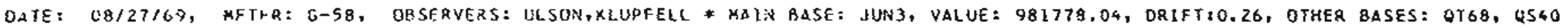

\begin{tabular}{|c|c|c|c|c|c|c|c|c|c|c|c|c|c|c|c|c|c|c|c|c|}
\hline & & & & l. 3 & 3.98 & A & \# & 0 & 18 & C & & 0804 & 981779.57 & $\mathrm{~B}$ & & $-14 \cdot 4$ & -15.1 & 4 & -15.0 & * QT5B \\
\hline N3 & AUKP & $* 58$ & 23.13 & 134 & .35 & A & * & 0 & 21 & $N$ & 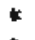 & 0810 & 981778.04 & A & * & -15.4 & -16.2 & 4 & -16.1 & * JUN3 \\
\hline 15 & & & 21.75 & 134 & 41.71 & 4 & - & 0 & -11 & $c$ & * & 0900 & $98\{78\} .38$ & D & * & -13.2 & -12.8 & 4 & -12.6 & -0515 \\
\hline 516 & & $* 50$ & 20.88 & 134 & 41.40 & 4 & * & 0 & -11 & c & * & 0907 & 981779.20 & 0 & $*$ & $-14=2$ & -13.8 & 4 & -13.9 & - 9s16 \\
\hline 17 & & \&58 & 19.84 & 134 & 43.82 & $\Delta$ & * & 0 & -11 & C & * & 0920 & 981775.17 & 0 & * & -16.9 & -16.5 & 4 & -16.5 & * osi7 \\
\hline 18 & LITE & * 58 & 20.79 & 134 & 45.02 & A & $\star$ & 13 & 23 & $\mathrm{c}$ & * & 0933 & 981776.44 & 0 & $*$ & -13.7 & -14.5 & 4 & -14.5 & $+0 \$ 18$ \\
\hline 519 & & $\begin{array}{r}-58 \\
\end{array}$ & 22.25 & L34 & 48.61 & A & * & 0 & -9 & c & * & 0951 & 981761.13 & 0 & $*$ & -16.0 & -13.6 & 4 & -13.7 & - 2519 \\
\hline 20 & & - 58 & 23.75 & 134 & 49.41 & $A$ & * & 1 & -6 & $c$ & " & 1007 & 981761.17 & 0 & 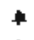 & -15.7 & -15.4 & 4 & -15.4 & $-\quad 0520$ \\
\hline$\Rightarrow$ & & $* 58$ & 24.89 & 134 & 50.26 & A & * & 0 & -7 & 5 & 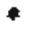 & 1016 & 981781.55 & $D$ & - & -16.9 & -16.6 & 4 & $-16+7$ & $* 0521$ \\
\hline 2 & & * 58 & 26.12 & 134 & 49.03 & $A$ & * & 0 & -5 & $\mathrm{c}$ & * & 1027 & 981788.77 & D & $*$ & -11.2 & -1.1 .0 & 4 & -21.0 & $-\operatorname{os} 22$ \\
\hline 23 & & - 58 & 27.67 & 13.4 & 49.40 & $A$ & * & 1 & -3 & $c$ & 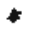 & 1044 & 981788.32 & D & $*$ & -13.5 & -13.4 & 4 & -13.4 & * Q523 \\
\hline$\$ 24$ & & * 58 & 26.43 & 134 & 54.60 & $A$ & $\bullet$ & $l$ & -2 & c & * & 1058 & 981782.34 & 0 & * & -17.7 & -17.6 & 4 & -17.6 & - $\cos 26$ \\
\hline 5 & & - 58 & 27.75 & 134 & 53.40 & A & $\#$ & 0 & -2 & c & $\bullet$ & 1111 & $98178 \mathrm{~L} .29$ & D & $*$ & -20.5 & -20 & 4 & -20 & $+9 \$ 25$ \\
\hline 6 & & $* 58$ & 29.26 & 134 & 54.93 & A & + & 1 & l & C & * & 1125 & 981781.66 & $D$ & $*$ & -21.9 & -21.9 & 4 & .9 & - 0s26 \\
\hline 527 & & +59 & 28.97 & 134 & $57.1 \%$ & $A$ & $*$ & 2 & 3 & C & 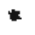 & 1137 & $9 B 1785.38$ & 0 & * & -17.6 & -17 & 4 & .7 & -0527 \\
\hline 28 & & 659 & 30.08 & 134 & 58.00 & $\Delta$ & * & t & 4 & $c$ & & 1154 & $98\} 786.04$ & 0 & $*$ & -18.4 & -18.5 & 4 & -18.5 & - QS2B \\
\hline $5>0$ & & $\neq 58$ & 30.39 & 135 & 0.11 & 4 & $*$ & 0 & 4 & E & 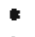 & 12 & 9817 & b & * & -15.2 & -1 & 4 & -15.4 & - 0529 \\
\hline 530 & & $\neq 58$ & 31.39 & 135 & 1.65 & $A$ & * & I & 6 & $c$ & * & 1218 & 981 & 0 & - & -27.1 & -27 & 4 & -3 & - 0530 \\
\hline 31 & LITE & $\neq 5 d$ & 32.43 & $1+5$ & 2.70 & $\Delta$ & * & 42 & 48 & c & * & 1240 & 9817 & D & * & -29.6 & -31 & 4 & -3 & $1 \cos 31$ \\
\hline$\$ 32$ & & - 58 & 32.75 & [134 & $55.2 \%$ & $A$ & * & 2 & 11 & $\varepsilon$ & * & 1338 & 981785.89 & 0 & * & $-2 I=4$ & -21 & 4 & -21.8 & -0532 \\
\hline 2533 & & * 58 & 33.36 & $1 \pm 4$ & 53.13 & $\Delta$ & * & 1 & 10 & $\mathrm{c}$ & * & 1350 & $98[794.24$ & o & + & -14.0 & -14.4 & 4 & $-14,3$ & $\cdot 0533$ \\
\hline 9168 & BASE & * 58 & 34.57 & 134 & 53.46 & $A$ & $*$ & 3 & 9 & c & 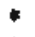 & 1401 & 4.16 & A & $*$ & -15.8 & $-16 \cdot 1$ & 4 & -16.1 & + QT68 \\
\hline 4534 & & * 59 & 34.61 & 134 & 55.61 & $\mathrm{~A}$ & $*$ & 1 & 10 & c & t & $14 L 3$ & 981798.05 & D & * & -11.9 & -12 & 4 & -1 & -0534 \\
\hline 2535 & & $\div 58$ & 35.79 & 134 & 54.40 & $A$ & * & 2 & 11 & c & & 1424 & 981800.87 & 0 & $*$ & -10.5 & -10 & 4 & -1 & $* 0$ \\
\hline 0536 & & - 58 & 36.65 & 134 & 56.30 & $\mathbf{A}$ & $*$ & 2 & 11 & $\mathrm{c}$ & 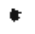 & 1442 & $98 \ 80 ! .67$ & 0 & $*$ & -10.9 & -11 & 4 & $-11 \cdot 3$ & - 0 \\
\hline 2537 & & * 58 & 37.93 & 134 & 56.65 & A & * & 1 & 10 & C & $\div$ & 1455 & 9818 & D & * & -8.3 & -8 & 4 & -8.7 & +0537 \\
\hline 0538 & & * 58 & 39.10 & $1 \$ 4$ & 58.10 & A & . & 2 & 20 & c & * & 1512 & 901799.82 & 0 & * & -16.3 & -16 & 4 & -12 & * os 30 \\
\hline 9539 & & * 58 & 40.30 & 134 & 50.08 & $A$ & $*$ & 3 & I! & $\mathrm{C}$ & * & 1527 & 981796.16 & 0 & $\bullet$ & -21.3 & -21 & 4 & $-21 . t$ & +0539 \\
\hline 0540 & BASE & - 58 & 40.78 & 134 & 56.09 & $\mathbf{A}$ & * & 15 & 22 & c & 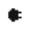 & 1546 & 981790.15 & B & $\bullet$ & -26.9 & -27 & 4 & -2 & - Q540 \\
\hline 0541 & & $* 58$ & 39.52 & 134 & 54.36 & $A$ & * & 1 & 6 & $c$ & $*$ & 1612 & 981793.04 & D & * & -23 & -24 & 4 & & - $0 \$ 41$ \\
\hline $\cos 42$ & & - 58 & 42.07 & 134 & 56.52 & A & * & 2 & 6 & $t$ & * & 1630 & 981788.27 & 0 & * & $-32 \cdot 0$ & -32.2 & 4 & $-32,2$ & $+\cos 42$ \\
\hline 3543 & & $\cdot 58$ & 43.59 & 134 & 56.46 & $A$ & 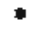 & 2 & 5 & c & * & 1645 & $9 \mathrm{at} 782.2 \mathrm{E}$ & 0 & $*$ & -40.1 & -40.3 & 4 & -40.3 & -0543 \\
\hline 0544 & & +58 & 44.93 & 134 & 55.89 & $\mathbf{A}$ & - & $\mathrm{l}$ & 4 & $c$ & - & 1657 & 961776.29 & 5 & + & -48.0 & $-4 B .2$ & 4 & & 10544 \\
\hline as 45 & & $+5 \mathrm{\theta}$ & 46.41 & 114 & 55.90 & A & 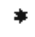 & 3 & 5 & c & * & 1709 & 981767.49 & 0 & * & -56.7 & -58.9 & 4 & -58.9 & -0545 \\
\hline 4546 & & - 54 & 47.95 & 134 & 57.93 & $A$ & * & 1 & 2 & C & * & 1726 & 981771.19 & D & - & -57.3 & -57.4 & 4 & & - 0546 \\
\hline US47 & & - 58 & 47.54 & 114 & 59.80 & $\mathbf{A}$ & & 2 & 2 & $c$ & * & 1740 & 981781.37 & 0 & 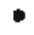 & -46.6 & -46.7 & 4 & & $+\operatorname{0s} 47$ \\
\hline 3548 & & * 5B & $7 x .47$ & 135 & 1.35 & $A$ & . & 3 & 1 & c & * & 10.24 & 981782.60 & 0 & $*$ & -44.0 & $-44=1$ & 4 & -44.1 & $-0 \$ 48$ \\
\hline 0549 & & - 56 & 45.31 & 135 & 1.06 & 4 & - & 1 & -2 & $\mathrm{c}$ & * & 1834 & 981782.37 & 0 & $*$ & -43.0 & -42.9 & 4 & -42.9 & -0549 \\
\hline Q550 & & * 58 & 44.02 & $1+5$ & $1 * 11$ & 4 & * & 1 & -3 & $\mathrm{c}$ & * & 1845 & 981781.06 & $D$ & 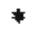 & $-42+7$ & -42 & 4 & -42.6 & -0550 \\
\hline $\cos 5 \mathrm{i}$ & & -58 & 45.86 & 135 & 3.91 & A & * & 3 & -2 & $\mathrm{c}$ & & 1907 & 981783.24 & D & * & -42.9 & -42.8 & 4 & -42.8 & * 0s51 \\
\hline $0 \$ 40$ & î & +59 & 40.79 & 154 & 56.09 & $\mathbf{A}$ & 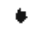 & 0 & 22 & $\mathrm{C}$ & * & 2019 & 981790.15 & B & 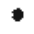 & -26.9 & -27.7 & 4 & -27.7 & - $0 \$ 40$ \\
\hline
\end{tabular}

METER G-SB HAO PROBABLE TARE OF +0.25 MGAL AT SOMETIME OLRING THE MORNING

WAIA_SLESISY

NUMHER OF $514 T$ I'INS: 41

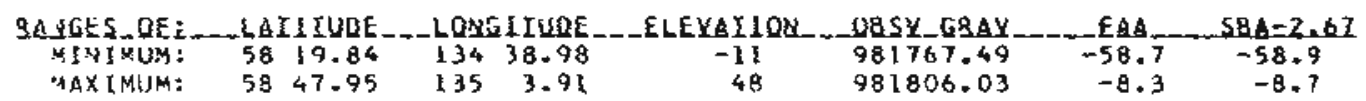


USGS GRAVIFY OATA FZUM: SUUTHEAST ALASKA. TRAYERSE: RETREAT N. PROJ CHJEF: BARNES. DATUM: BARNES I9TI DATA SET: AP54

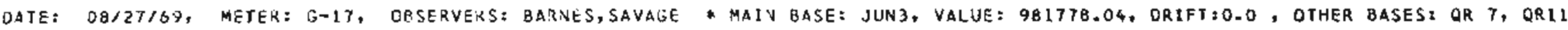

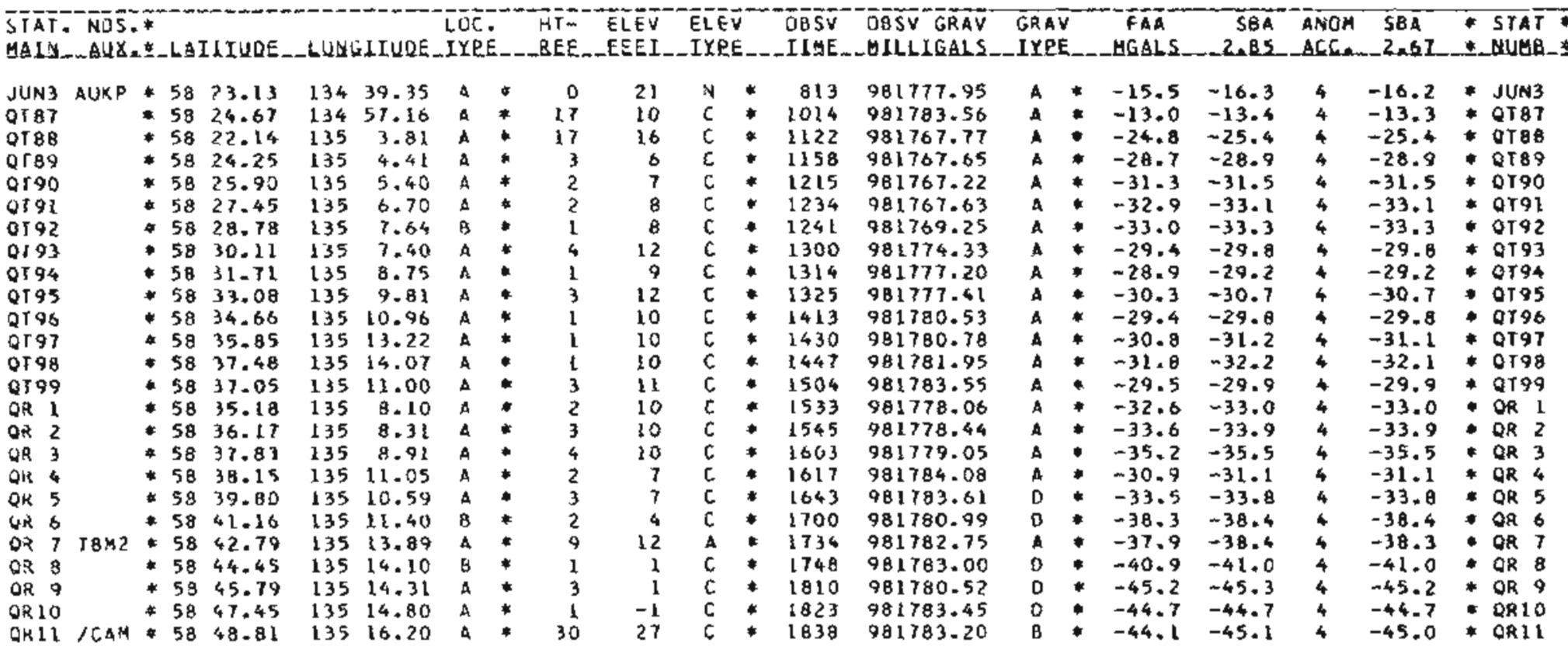

DAIA SilutaBY

NUHAER OF STATIJNS: 25

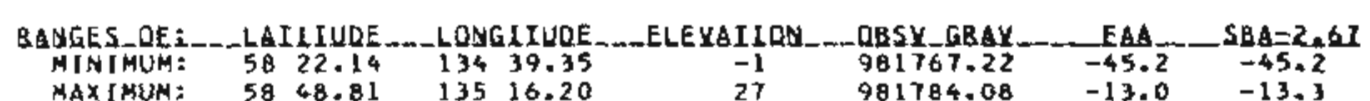




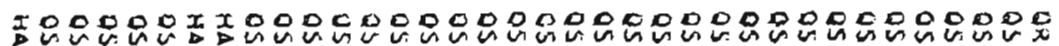

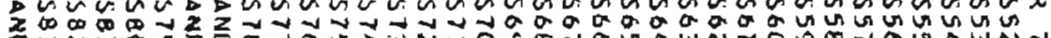

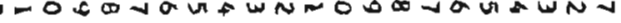

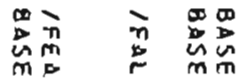

今

$i_{-1}^{i} \quad$ in

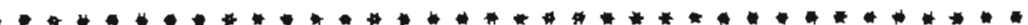

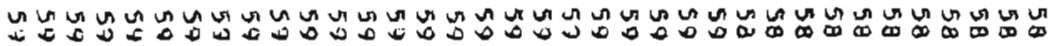

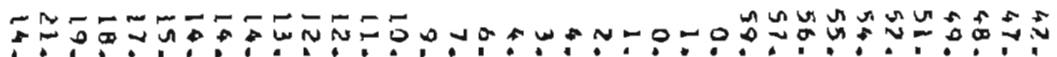

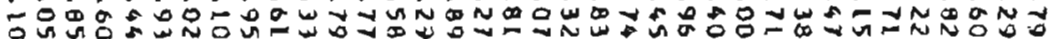

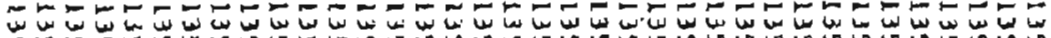

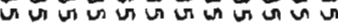

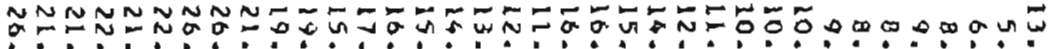

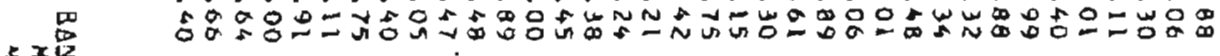

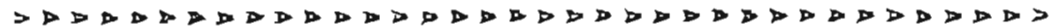

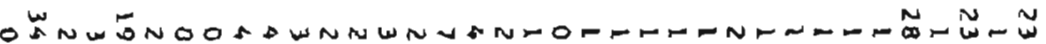

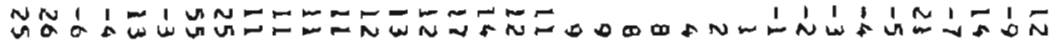

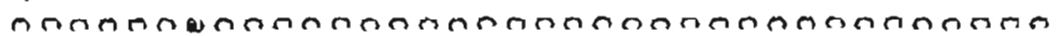

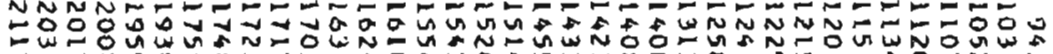

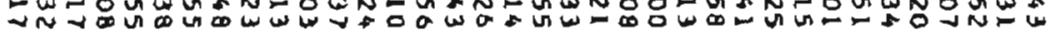

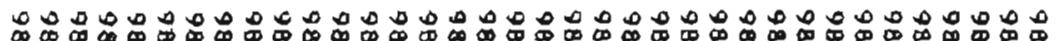

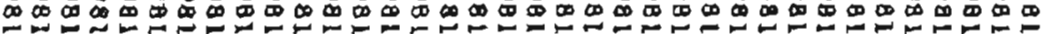

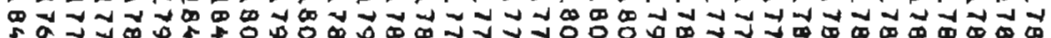

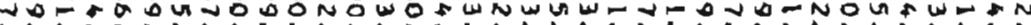

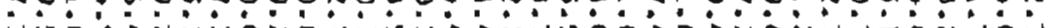

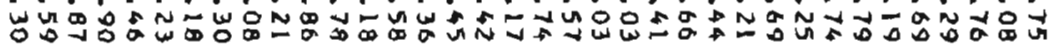

mantoso00000000000000000000000000x

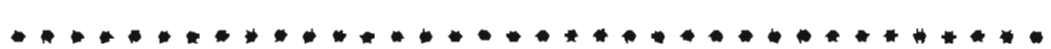

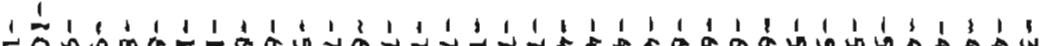

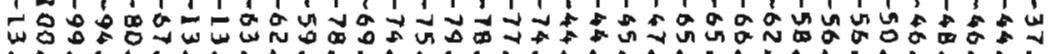

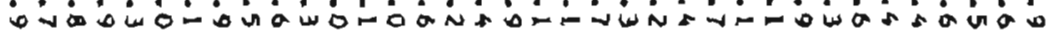
1

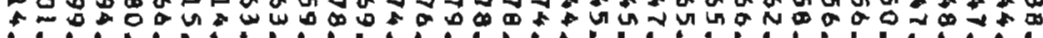

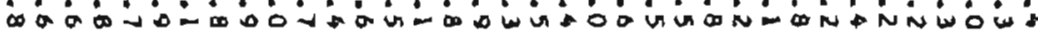

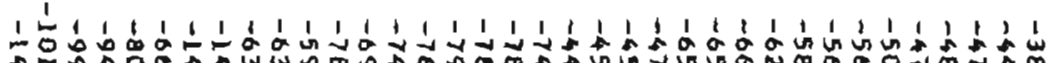

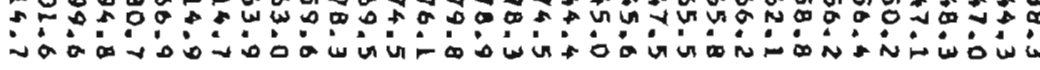

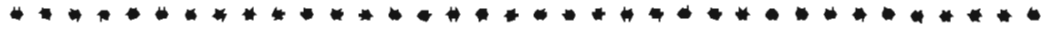

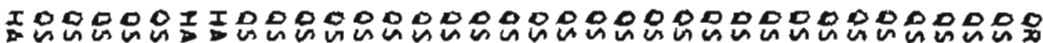

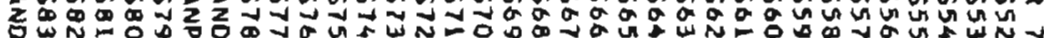




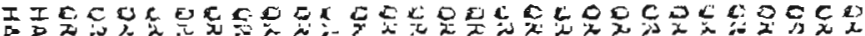

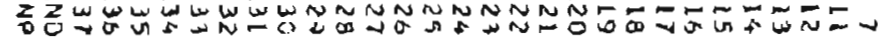

思

学

齐

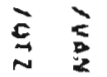

齐要

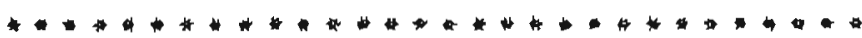

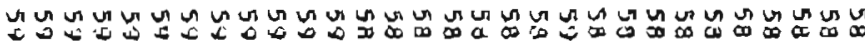

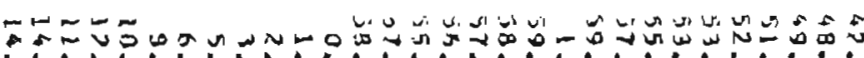

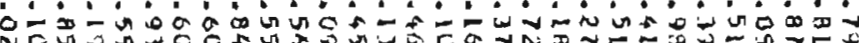

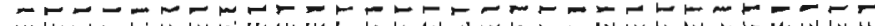

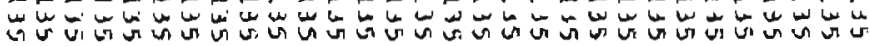
Uू 年

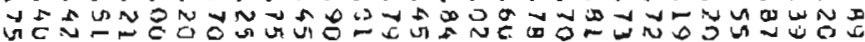
$\triangle D D D D M D D D D D D D D D D D D D D D F D D D D$

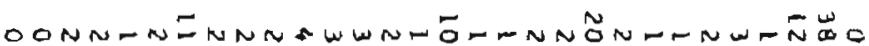

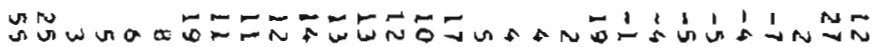

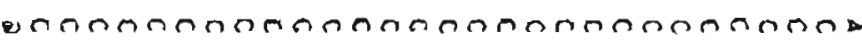

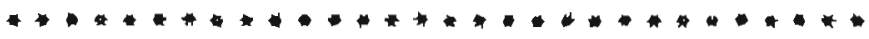

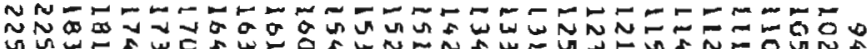

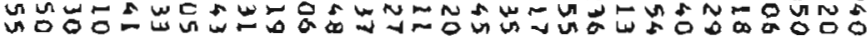

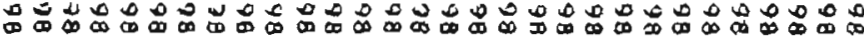

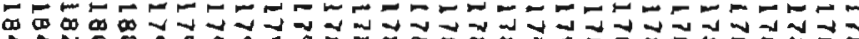

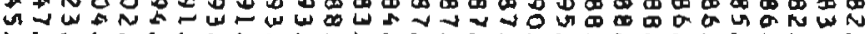

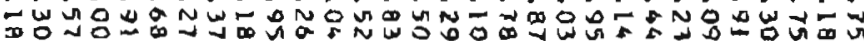
10000000000000300000000000000

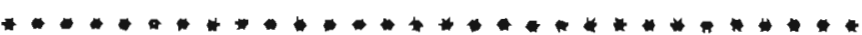

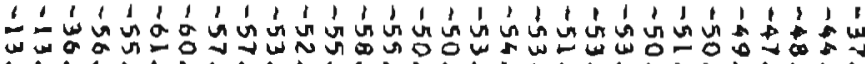

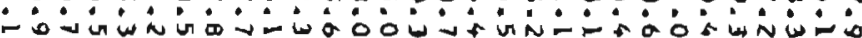

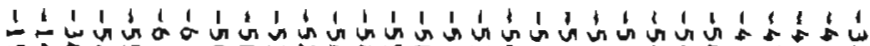

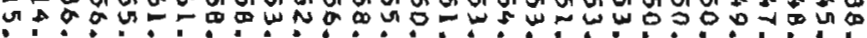
- Do vo in

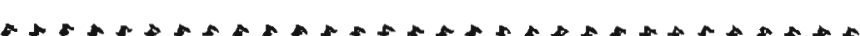

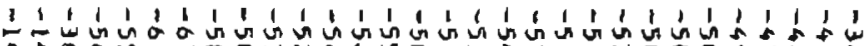

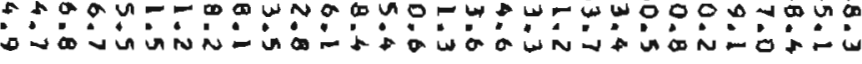

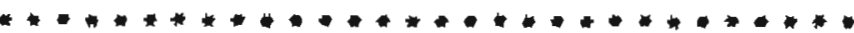

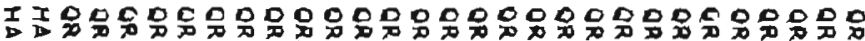

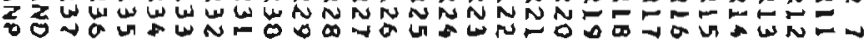




STAT. NISS."

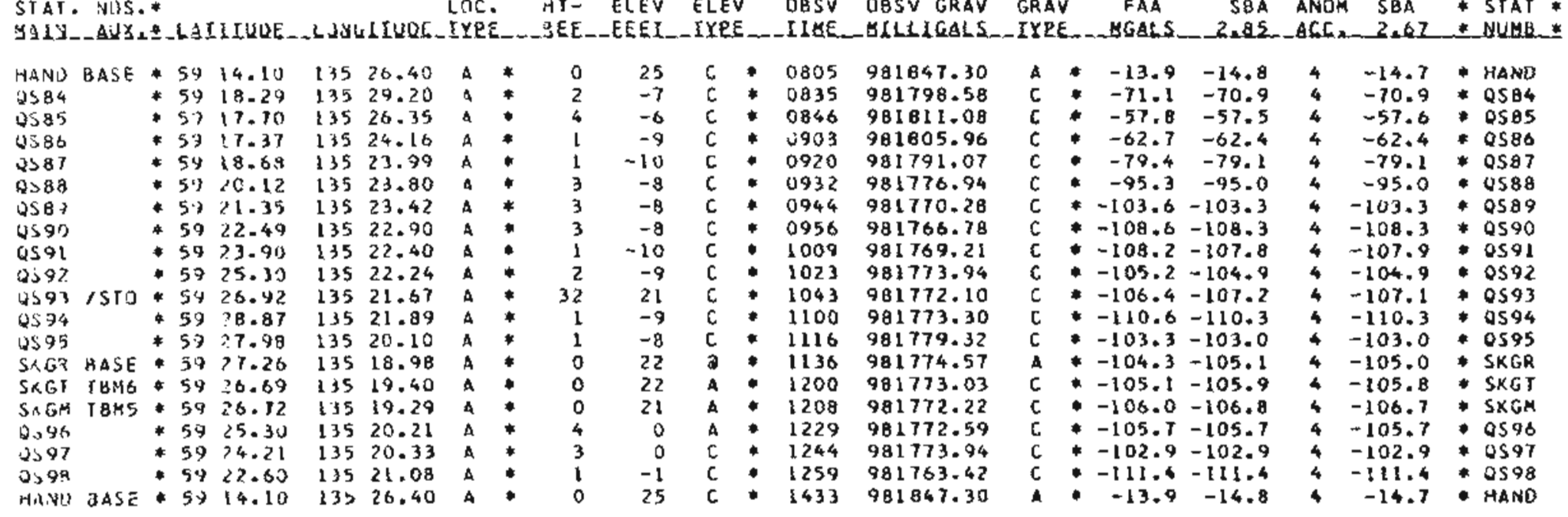

QAIA_SUGYASY

NUMEER UF STAFIYMS: 20

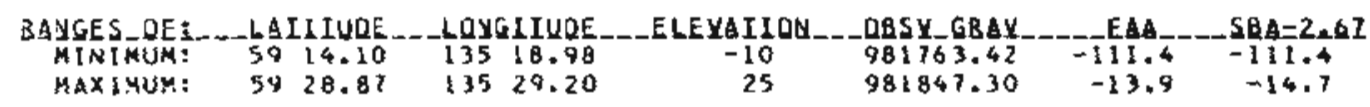

USGS GRAVITY DATA FRLM: SOUTHEAST ALASKA, TQAVERSE: MOOSE ALT PROJ CHIEF: baRNES DATUM: BARNES 1971 DATA SET: APSB

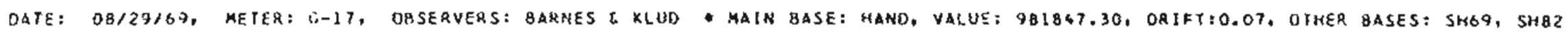

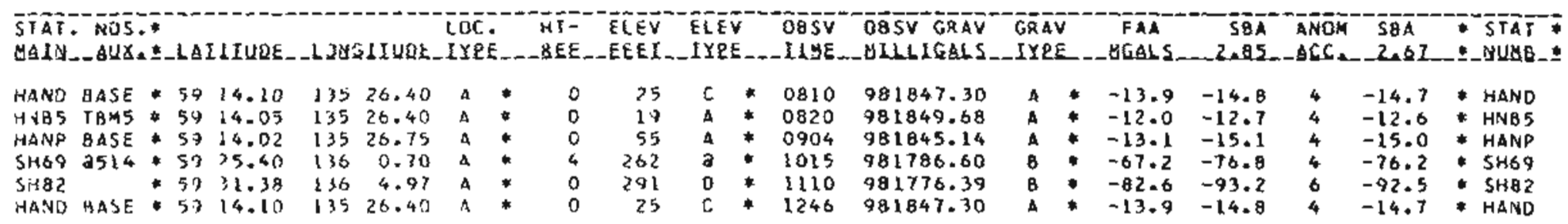

THAVEXSE USED PRIMARILY FOR ALTIMETRY ON CHILKAT RO B/L6/69 TRAVERSE

TAIA_Sigya3r

WUEER UF STATLUMS:

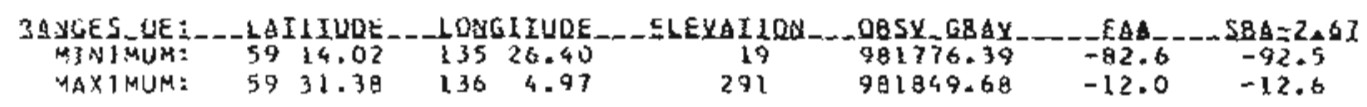




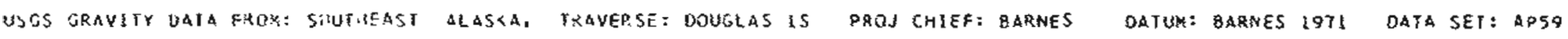

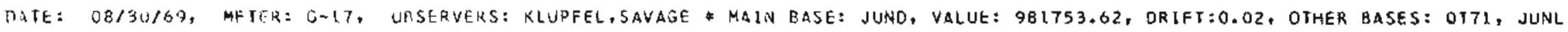

\begin{tabular}{|c|c|c|c|c|c|c|c|c|c|c|c|c|c|c|c|c|c|c|c|c|}
\hline $\begin{array}{l}5 T A T . \\
\text { dAS }\end{array}$ & $=\times 10$ & $=*$ & ruot & & 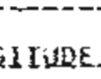 & LI! & & $1=$ & ET & & & $\begin{array}{l}\text { DESV } \\
\text { IILE. }\end{array}$ & $\begin{array}{l}085 V G R A V \\
\text { SILLIGALS }\end{array}$ & & & $\begin{array}{l}\text { FAA } \\
\text { GGALS }\end{array}$ & $\begin{array}{r}5 B A \\
2.95\end{array}$ & & $\begin{array}{l}584 \\
2.67\end{array}$ & 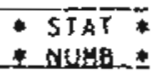 \\
\hline $\mathbb{N N L}$ & GASF & $* 5$ A & 21.63 & 134 & 34.94 & $\Delta$ & 4 & 0 & 20 & $E$ & * & 1100 & 981767.78 & A & $*$ & -23.8 & -24.5 & 4 & -24.5 & * JUNL \\
\hline UNO & DASE & * 5日 & 18.3 & 134 & 5.41 & $A$ & 中 & 0 & 17 & E & * & 7 & 3.62 & A & + & -33.7 & -3 & & -3 & JND \\
\hline 171 & BLM & D 59 & $\mid A .61$ & 134 & 3.01 & 4 & 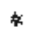 & 24 & 18 & c & $*$ & 12 & 7.53 & B & 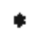 & -27.4 & -2 & 4 & & - 0T71 \\
\hline $1 \times 38$ & & $\neq 58$ & 15.62 & 134 & 21.50 & $A$ & 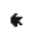 & i & -4 & $c$ & 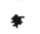 & 2228 & 901762.05 & c & * & -23.6 & -23.5 & 4 & $-23,5$ & - OR38 \\
\hline 23 & & - 58 & $14.7 \mathrm{~L}$ & 154 & .92 & $\mathbf{A}$ & 4 & 1 & -4 & c & * & 1239 & 2.53 & $c$ & * & $-21,9$ & -21.8 & 4 & -21.6 & - QR39 \\
\hline $28: 40$ & & $\Rightarrow 58$ & 13.49 & 134 & $\mathrm{I}$ & A & * & $\mathrm{t}$ & -2 & 6 & $*$ & La & 0.44 & c & $\star$ & -2 & -2 & 4 & -2 & 40 \\
\hline 41 & & 458 & 12 & 134 & 16.00 & $A$ & * & i & -2 & c & $\$$ & 1311 & 0.33 & $\mathrm{E}$ & - & -20 & $-\bar{z}$ & 4 & -2 & * QR 41 \\
\hline $2 \times 42$ & & $\begin{array}{l}-58 \\
\end{array}$ & $1 \mathrm{~L} .68$ & 134 & 17.71 & A & * & 2 & 1 & C & 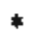 & 1327 & 2.06 & c & $\star$ & -17.8 & -1 & 4 & -1 & $+0 R 42$ \\
\hline 51743 & & * 58 & 11.99 & 134 & 20.39 & A & \# & 2 & 2 & C & * & 1342 & 1.75 & c & $*$ & -1 & & 4 & & - OR4 3 \\
\hline 0.444 & & - 58 & 12.59 & 134 & & $A$ & * & 1 & 4 & C & $*$ & 1418 & .14 & $c$ & * & -1 & -1 & 4 & -1 & - 0R44 \\
\hline 2845 & & $\Rightarrow 5 \mathrm{~B}$ & 17.17 & 134 & 24.67 & $\boldsymbol{b}$ & * & 2 & 5 & c & * & 1431 & 1.48 & c & * & -2 & -2 & 4 & -2 & - OR45 \\
\hline 0246 & & $\neq 53$ & 13.70 & 134 & 27,20 & 4 & $*$ & $i$ & 5 & c & $*$ & {$[44]$} & 2.89 & $\mathrm{C}$ & $*$ & -19.4 & & 4 & -19.5 & - OR46 \\
\hline 9847 & & * 58 & 13.10 & 134 & & A & 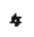 & 12 & 18 & c & * & 1458 & .87 & c & $*$ & $-i$ & -2 & 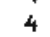 & .0 & * QR4 4 \\
\hline 9448 & & +50 & 13.46 & 134 & 37.80 & A & * & 2 & 9 & c & * & 15 & 57.44 & $c$ & * & -2 & -2 & 4 & -2 & $\because 0 R 48$ \\
\hline 0244 & & * 58 & 13.92 & 134 & 35.27 & A & * & $i$ & 4 & c & $*$ & 1525 & 901755.91 & $\mathrm{c}$ & * & -26.3 & -26 & 4 & -20 & * QR 49 \\
\hline 0850 & LIE & +55 & 14.97 & 154 & 33.62 & $\Delta$ & . & 5 & 13 & $\varepsilon$ & $*$ & $154 \mathrm{I}$ & 982759.59 & c & - & & & 4 & -24.0 & +0850 \\
\hline 6251 & & +58 & 36.44 & 134 & & $\mathbf{A}$ & $*$ & 2 & 10 & $c$ & $*$ & 10 & 3.93 & 8 & - & -2 & -21.9 & 4 & -21.9 & - Gr 51 \\
\hline Junil & BA $5 \varepsilon$ & +58 & 18.31 & 154 & 25.41 & $A$ & - & 0 & 17 & $\mathrm{c}$ & * & 1706 & 481753.62 & A & * & -33.7 & -34.4 & 4 & -34.3 & * JUNo \\
\hline
\end{tabular}

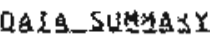

KUMAER JF STATIUNS: is

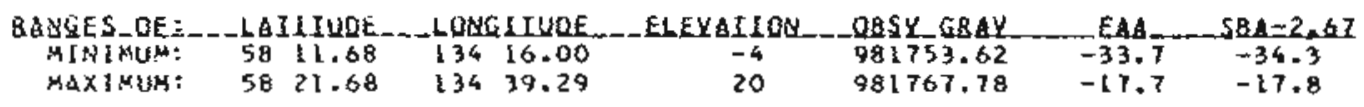




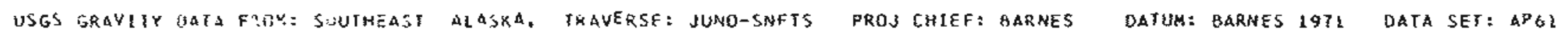

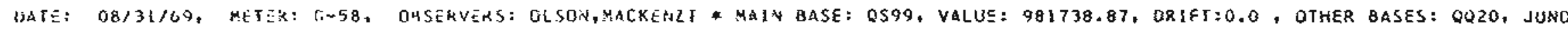

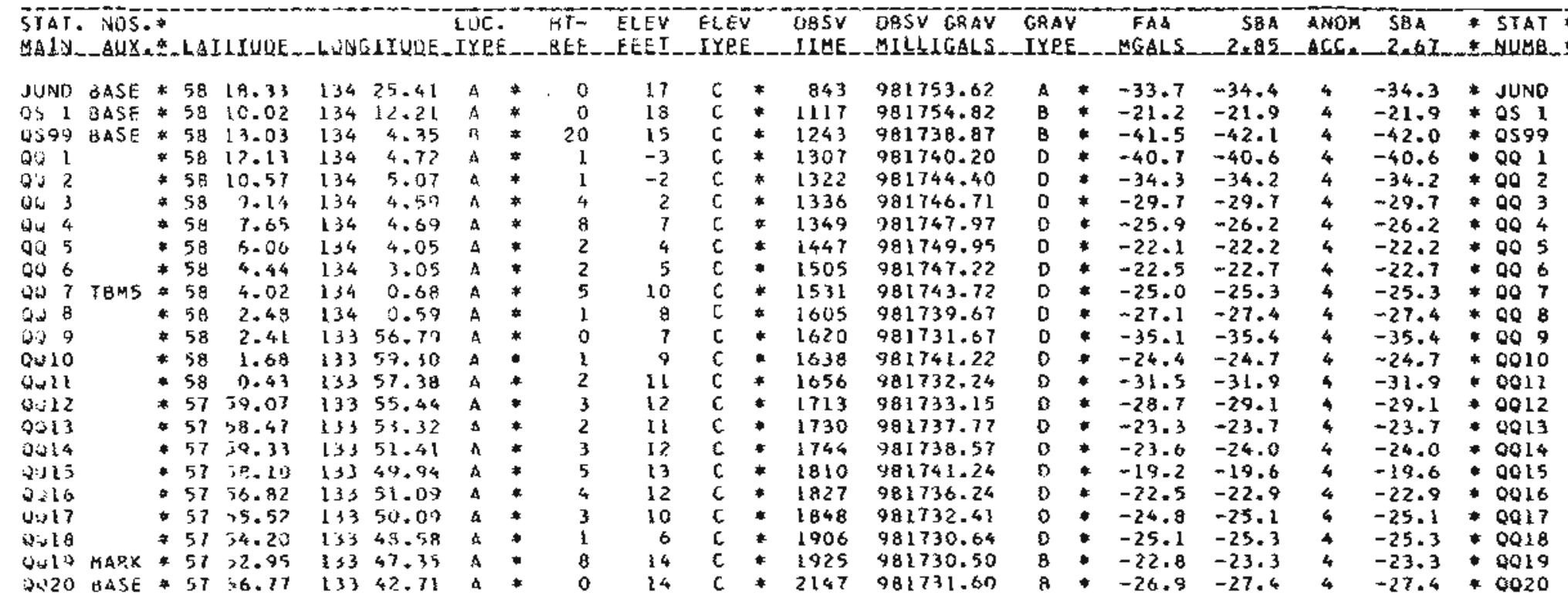

OA IA_SUSHASY

NUMMER DF STATIONS: 23

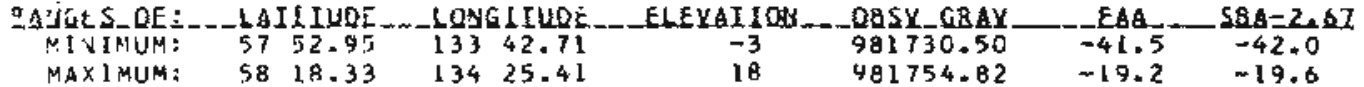




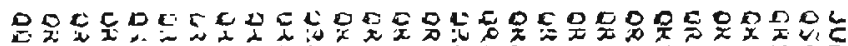

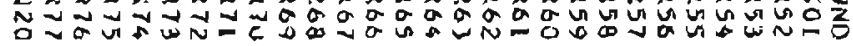
a

藏宾 $\vec{x} \quad \stackrel{i n}{c}$

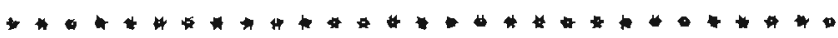

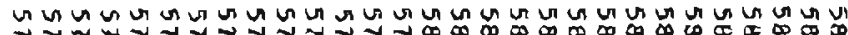

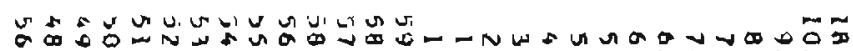

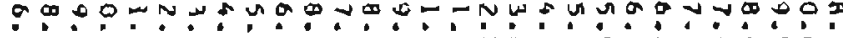

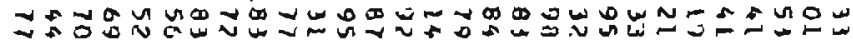

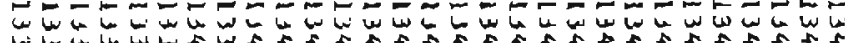

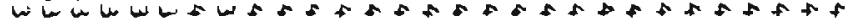

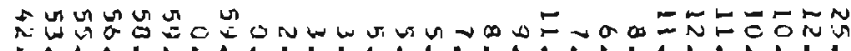

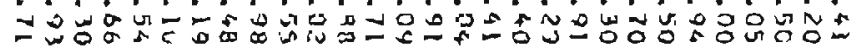

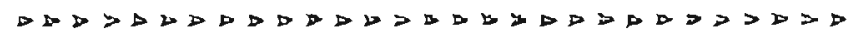

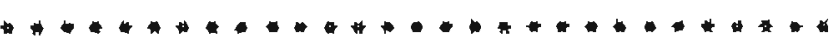

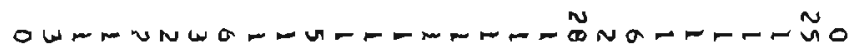

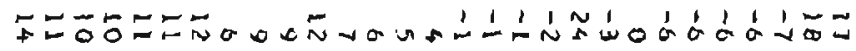

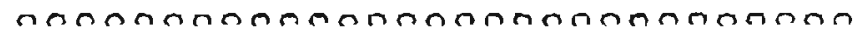

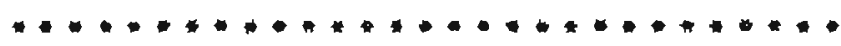

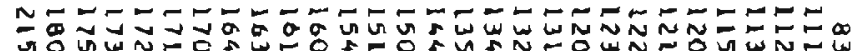

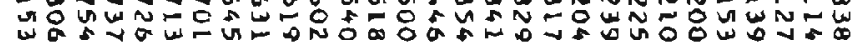

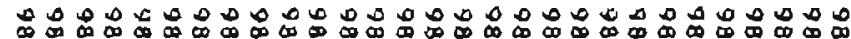

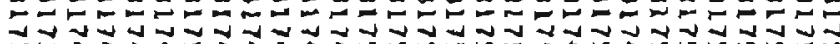
w ? ש

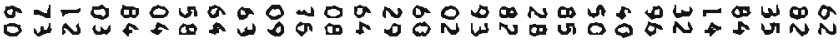

$\therefore 0000000000000000000000000 x$

$\ldots \ldots+\ldots+\ldots \ldots+\ldots \ldots+\ldots \ldots+\ldots+\ldots+\ldots$

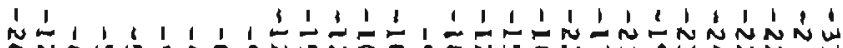

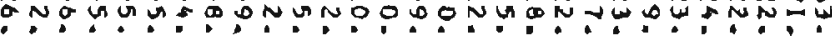

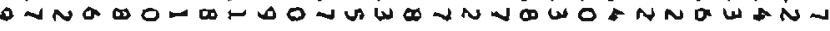

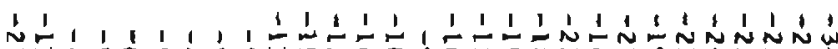

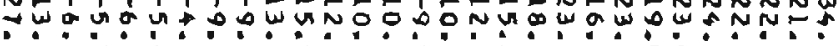

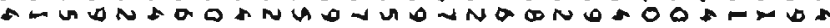

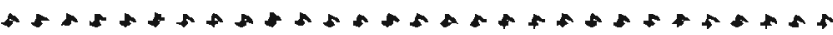

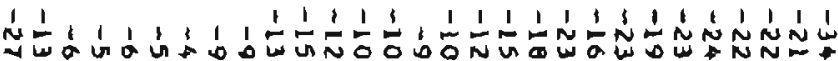

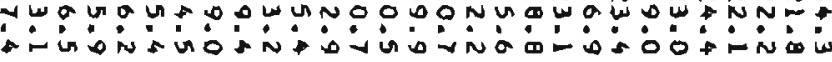

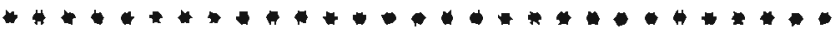

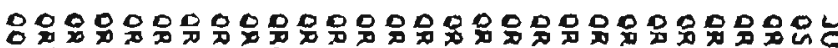

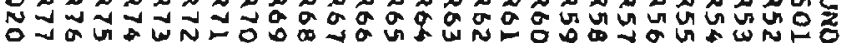




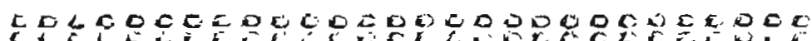

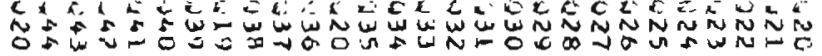
罢 索公

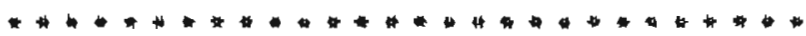

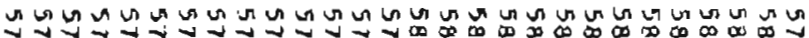

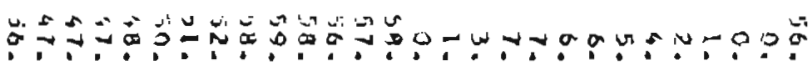
$\checkmark \Xi \cong$

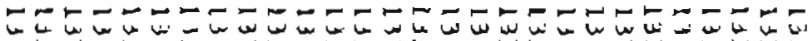

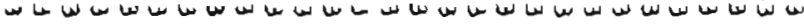

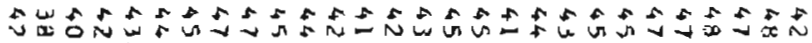

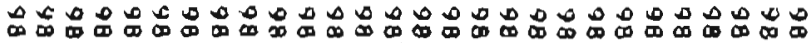

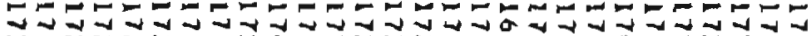
W

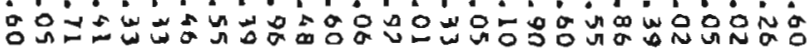

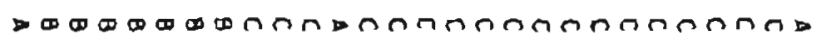

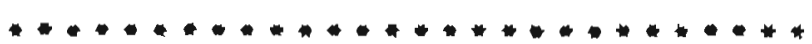

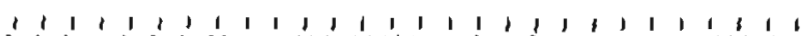

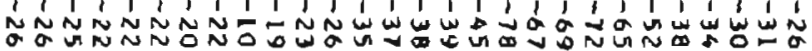
o

'́ñ

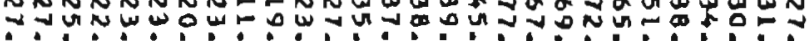

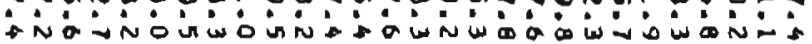

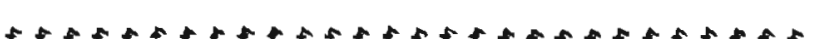

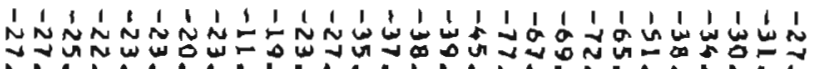
- Nir ín

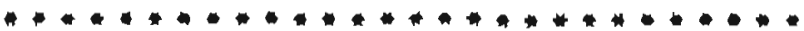

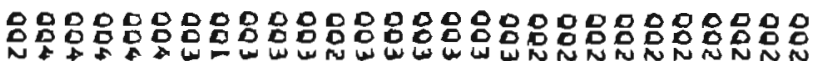

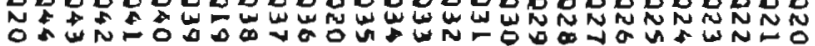




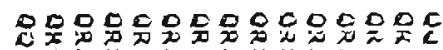

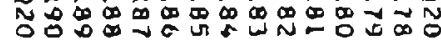

皮

$\underset{\pi}{x}$

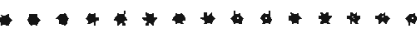

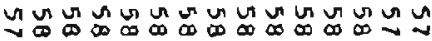

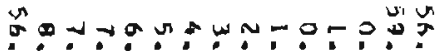

$\checkmark$ ป

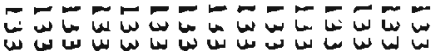
ANNNNNNWM NON N UNN zu va

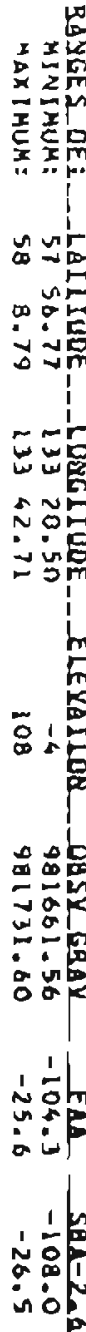

$D D D D D D D D D>D$

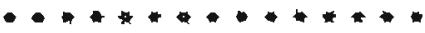

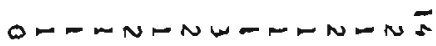

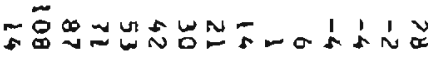

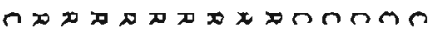
$\ldots \ldots \ldots \ldots$

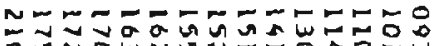

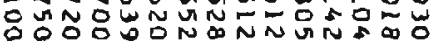

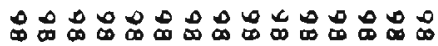

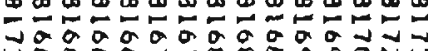
山a

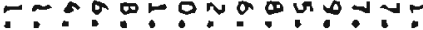

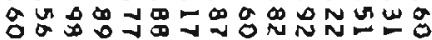
10000000000000

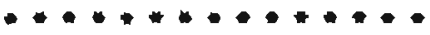

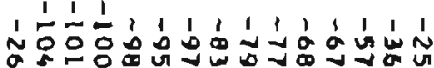

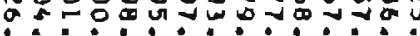
० ज

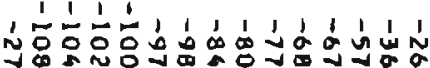
nog0000000

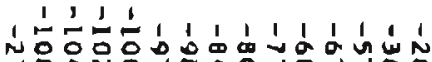

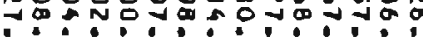
rovo:onanNonum

-

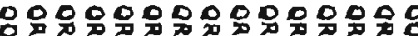

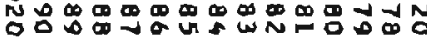

- $+\cdots+\cdots$

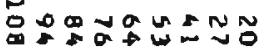

1 - isión: is 


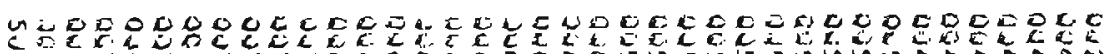

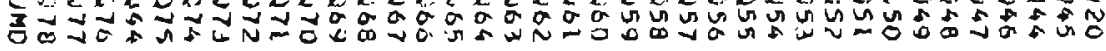
点

$$
\text { 胥 }
$$

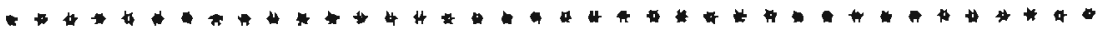

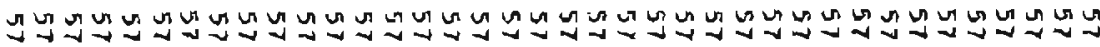

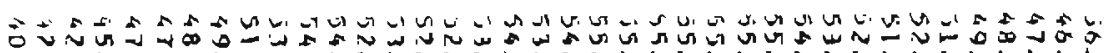

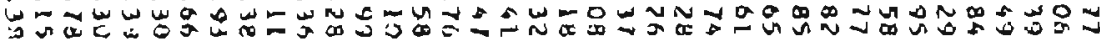

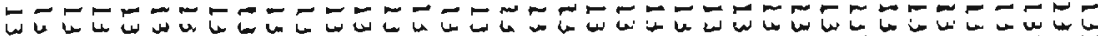

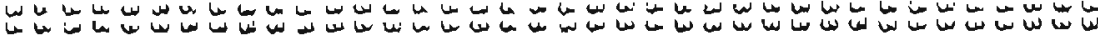

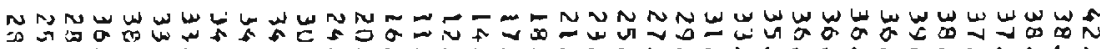

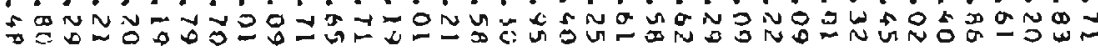

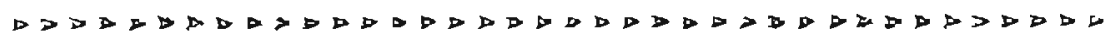

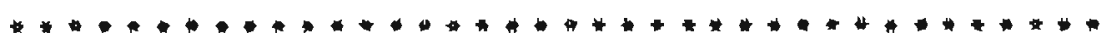

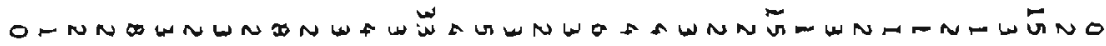
约

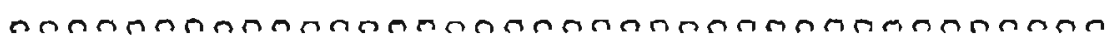

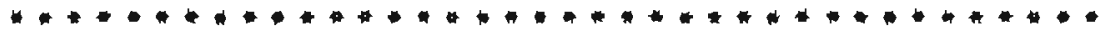

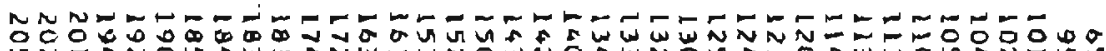

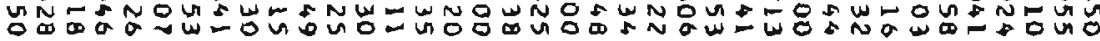

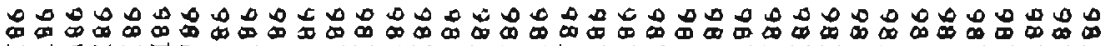

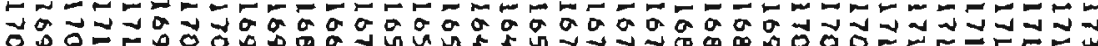

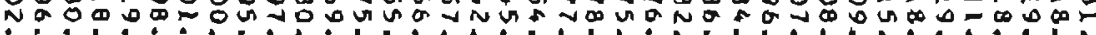

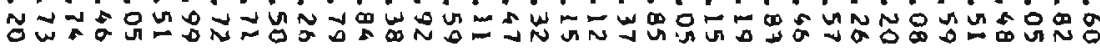

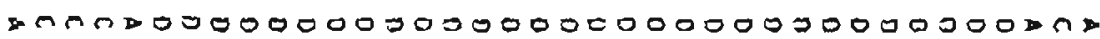
$\ldots \ldots \ldots \ldots \ldots \ldots \ldots \ldots \ldots \ldots \ldots \ldots \ldots \ldots \ldots+\ldots \ldots+\ldots \ldots$ 1 1 1 1 1 1 1 1 1 1 1 1 1 1 1 1 1 1 1 1 1 1 1 1 1 1 1 1 1 1 1 1 1 1 1 1

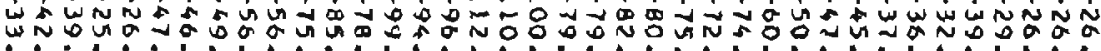

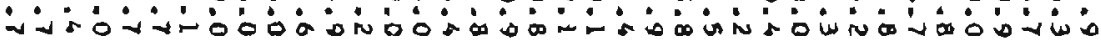

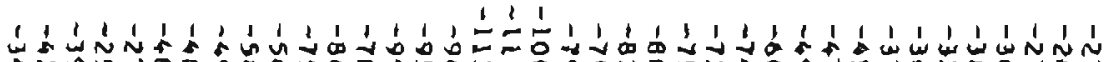

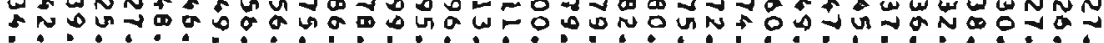

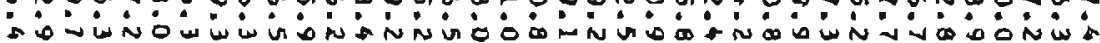

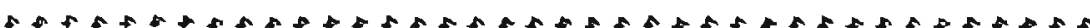

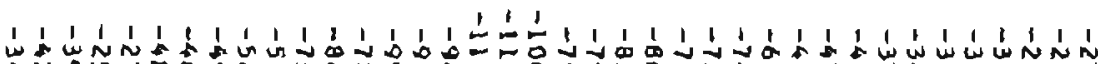
눈

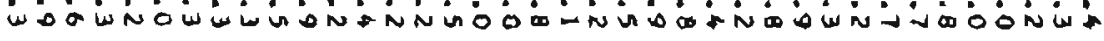

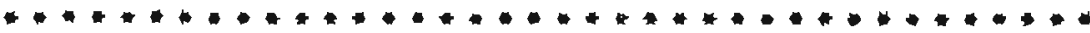

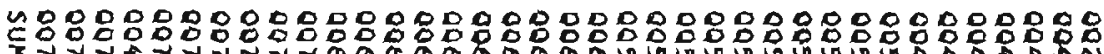

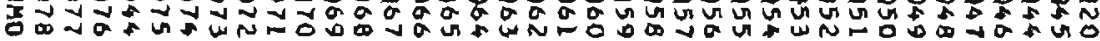




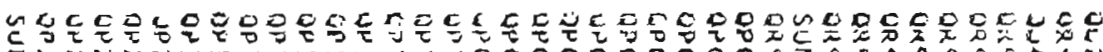

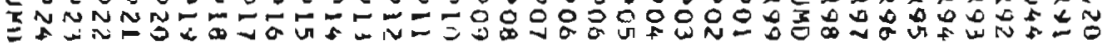
a in

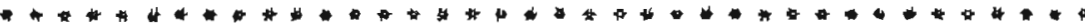

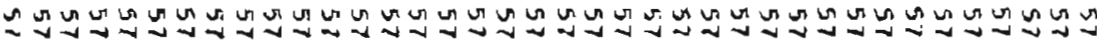

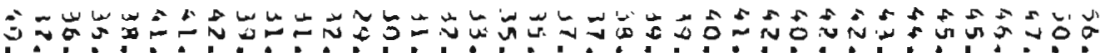

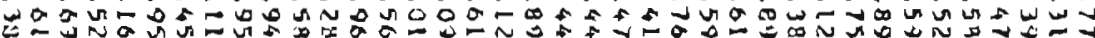

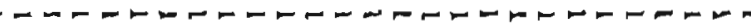

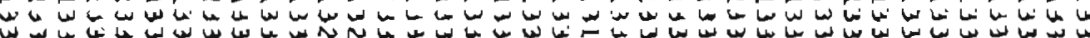

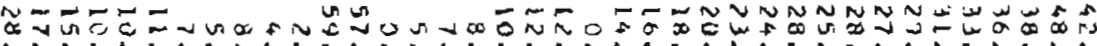

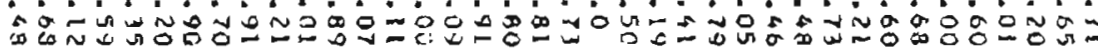

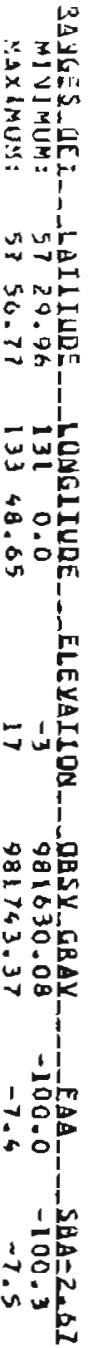
$D D \rightarrow D D D D D D D D D D D D D D D D D D D D D D D D D D D D$ $\ldots+\ldots \ldots+\ldots+\ldots \ldots+\ldots \ldots \ldots \ldots \ldots \ldots \ldots \ldots \ldots \ldots \ldots \ldots$

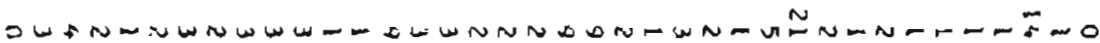

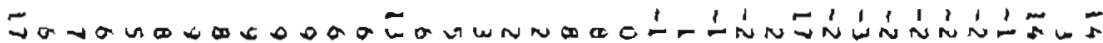

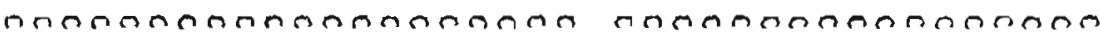

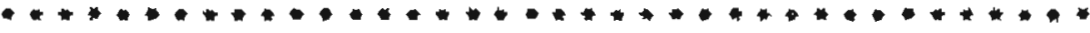

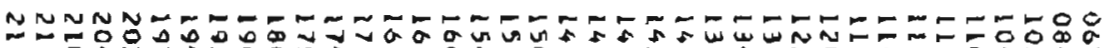

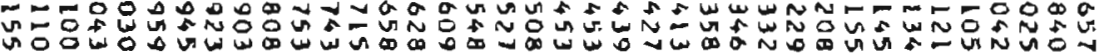

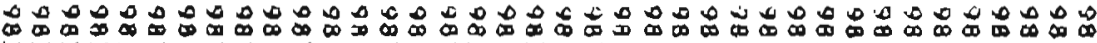

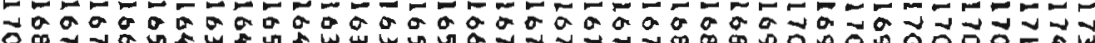

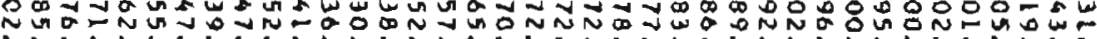

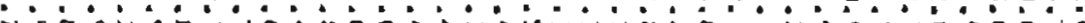

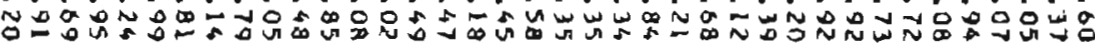

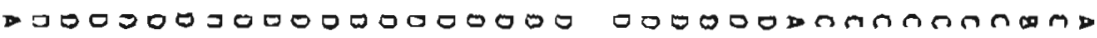

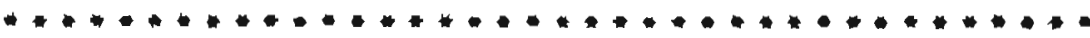

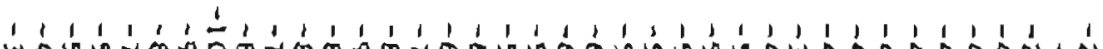

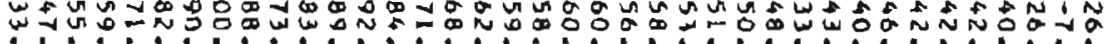

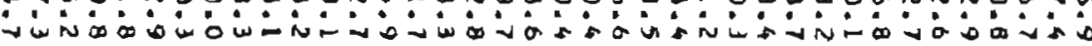

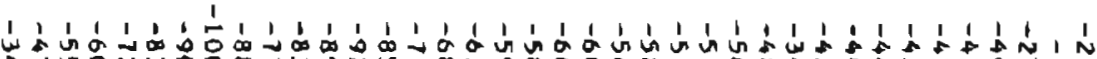

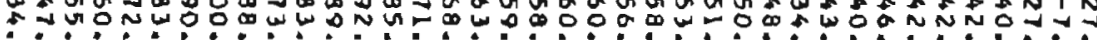

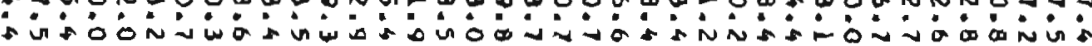
HA

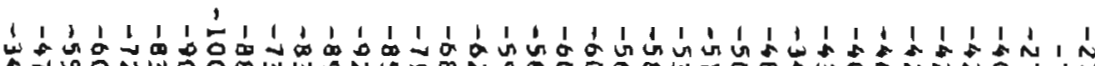
• 그유.

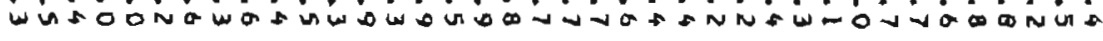

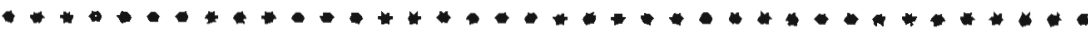

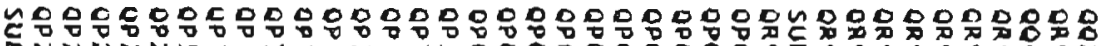

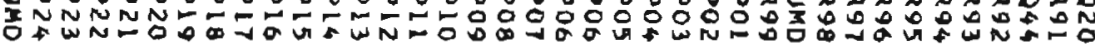




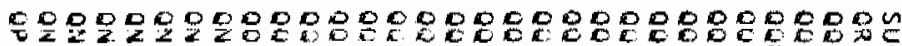

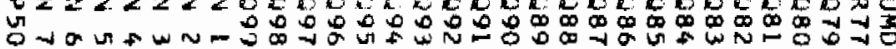
罯 $\stackrel{3}{\circ}$

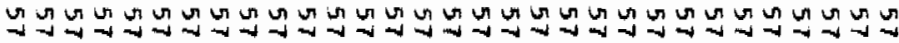
ज以UU

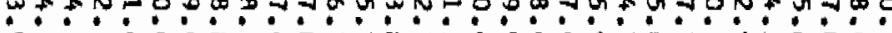

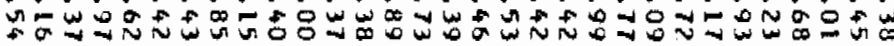

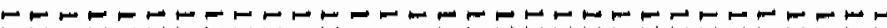

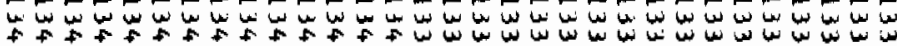
वワ

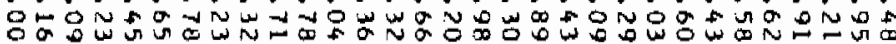

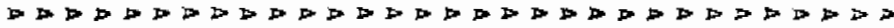

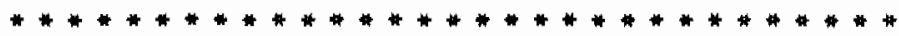

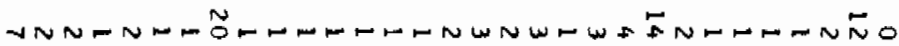

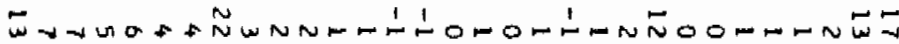

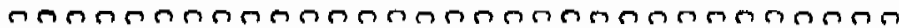

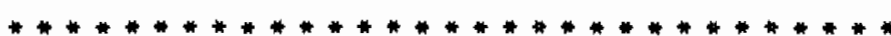

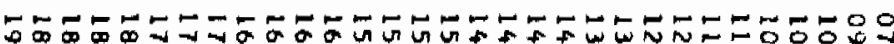

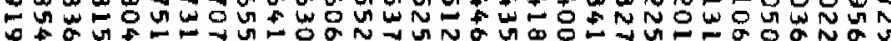

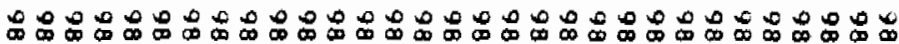

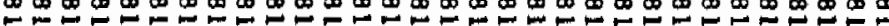
政UAN -

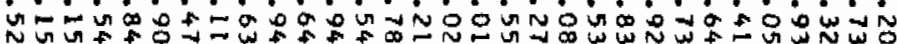
-

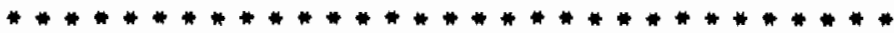

1

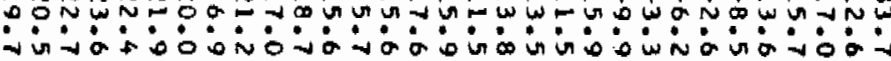

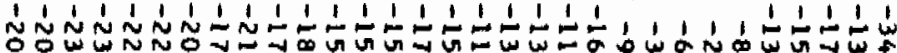

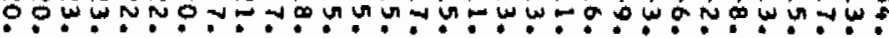
N

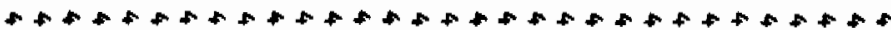

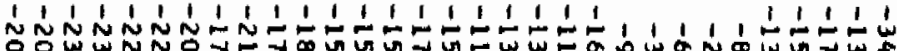

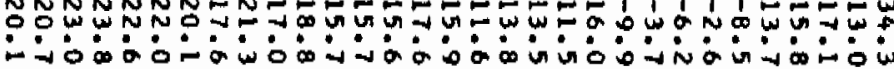

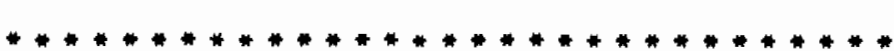

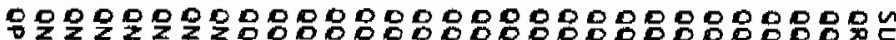
vavuwN- 


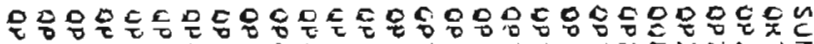

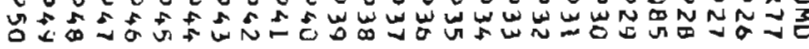

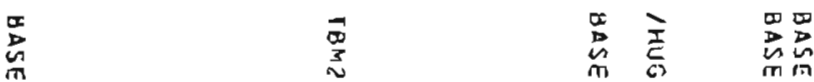

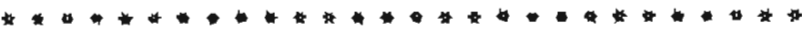

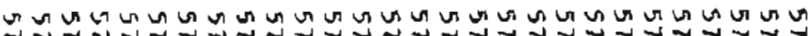
เด

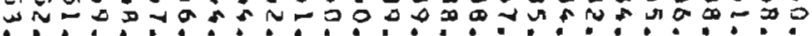

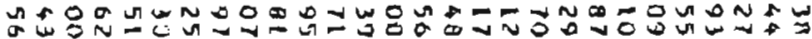

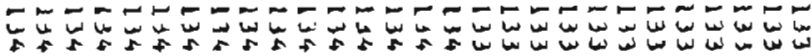

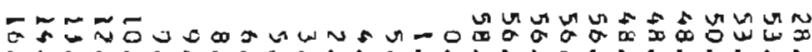

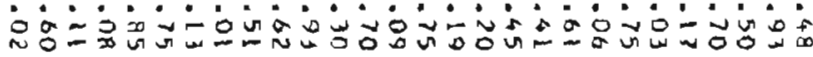

$\triangle P D D D D D D D D D D D D D D D D D D=D D$

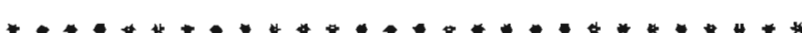

MRNNNMORNMNFMWRM-RMMONONMROO 西

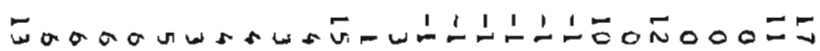

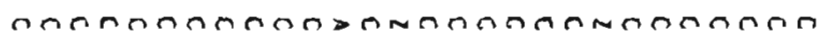

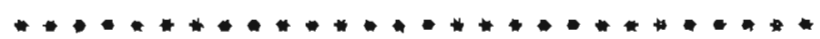

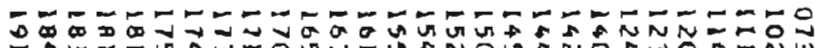

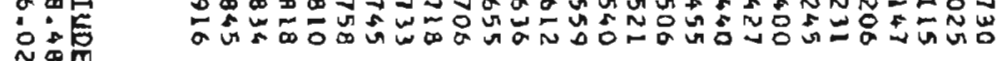

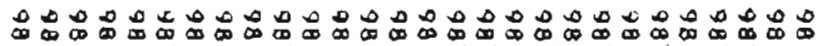

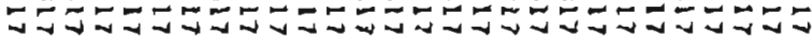

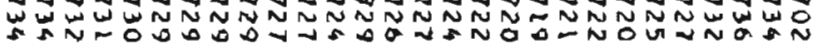

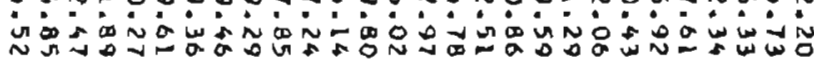

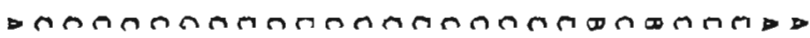

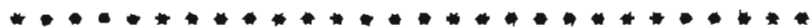

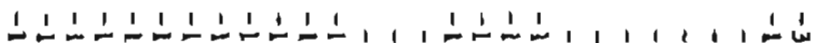

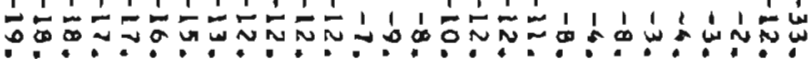

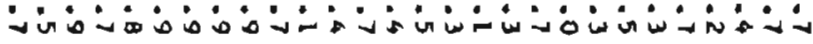

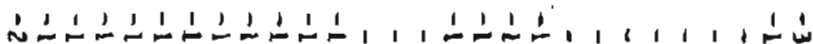

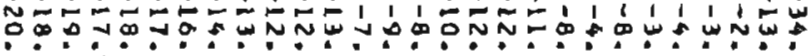

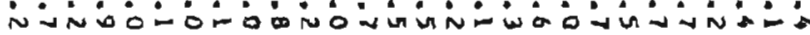

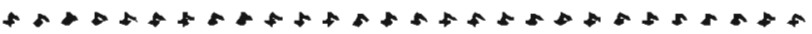

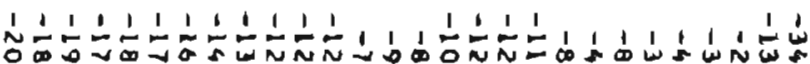

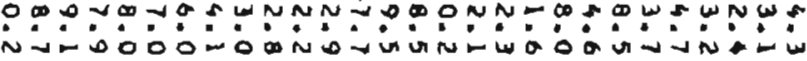

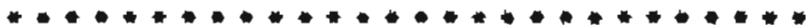

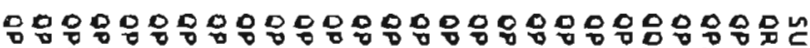

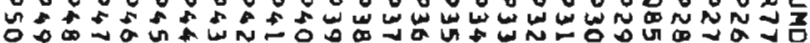


USGS GRAVITY DATA FRQY: SUUTHEAST ALASKA, YRAVERSE: SEYMOR-GAM PROJ CHIEF: BARNES DATUM: QARNES 197L DATA SET: APG9

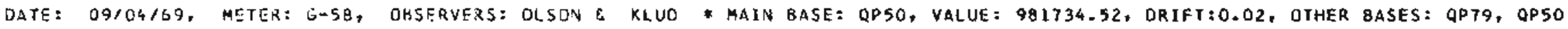

\begin{tabular}{|c|c|c|c|c|c|c|c|c|c|c|c|c|c|c|c|c|c|c|c|}
\hline $\begin{array}{l}\overline{S T A T} \\
\text { GAIS }\end{array}$ & $\begin{array}{r}n 0 \\
-40\end{array}$ & L & UD & D & 10 & $I$ & & & Ey & & $\begin{array}{l}B 5 V \\
14 E\end{array}$ & $\begin{array}{l}\text { OBSY } \\
\text { BLALIGALS }\end{array}$ & $\begin{array}{l}\text { GA } \\
\text { IX }\end{array}$ & & $\begin{array}{l}\text { FAA } \\
\text { MGALS }\end{array}$ & $\begin{array}{r}584 \\
2.85 \\
\end{array}$ & A & $\begin{array}{r}584 \\
-2.61\end{array}$ & 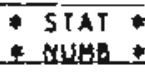 \\
\hline QP5O & BASE & 57 & 53.54 & 134 & 16.00 & $\mathbf{A}$ & $*$ & 0 & 13 & $c$ & 858 & 981734.52 & $A$ & • & -19.7 & -20.2 & 4 & -20.1 & QP50 \\
\hline 9408 & & 57 & & 134 & 9.39 & A & $r$ & 2 & 4 & $\varepsilon$ & 933 & 981735.31 & $c$ & $*$ & & & 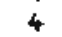 & & \\
\hline UN 9 & & -57 & 57.21 & 134 & 9.51 & A & $*$ & 2 & 4 & $\mathrm{C}$ & 948 & 981730.43 & $\mathrm{c}$ & $\bullet$ & -21.6 & -21.7 & 4 & $-21 \cdot 7$ & + ON 9 \\
\hline QNLO & & $\neq 57$ & 50.48 & 134 & 10.99 & $A$ & & 2 & 4 & $E$ & 958 & 981740.17 & C & * & -21.6 & -21.7 & 4 & -21.7 & - QN10 \\
\hline Q:411 & & +57 & 59.88 & 134 & 12.02 & A & * & 2 & 4 & c & 1011 & 781743.08 & C & * & -20.6 & -20 & 4 & -20.7 & Q QNIL \\
\hline QN & & * 58 & 0.99 & 134 & 14.33 & A & * & 2 & 4 & $c$ & 1023 & 981743.16 & C & * & -22.0 & -22.1 & 4 & -22.1 & - ON12 \\
\hline QN 1 & & $\neq 5 a$ & 2.37 & 134 & 14.98 & 4 & 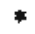 & 2 & 4 & C & 1036 & 981745.52 & r & $\star$ & -21.5 & -21 & 4 & $-21=6$ & - ON13 \\
\hline 0,14 & & $* 58$ & 1.11 & 134 & 11.39 & A & 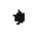 & 3 & 4 & $c$ & 10 & 901 & $c$ & - & -14.6 & -14 & 4 & -14.7 & - ONl4 \\
\hline ov & & * 58 & .26 & 134 & 12.79 & a & * & 1 & 2 & $c$ & 11 & 3 & 5 & * & $-16+8$ & -1 & 4 & -16.8 & - ONis \\
\hline $\sin 1$ & & * 58 & 3.75 & 134 & 15.00 & 4 & 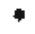 & 3 & 4 & $c$ & I I & 98 & c & $\bullet$ & & & 4 & -1 & - ga16 \\
\hline 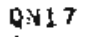 & & $* 58$ & 4.62 & 134 & 17.09 & A & * & 2 & 3 & $\boldsymbol{c}$ & 1133 & 48 & c & * & -2 & & 4 & & * 0 \\
\hline $0+18$ & & * 58 & 5.22 & 134 & 19.29 & A & 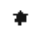 & 1 & 2 & c & 1144 & 98 & $r$ & $\star$ & -2 & -2 & 4 & -24.8 & - ONIB \\
\hline $0+19$ & & $* 5 B$ & 3.5 & 134 & 17.35 & A & $*$ & 1 & 1 & $c$ & 1200 & 981 & c & + & & & 4 & -23.5 & - ON19 \\
\hline QN20 & & 458 & .83 & 1,14 & .22 & 4 & $*$ & 2 & 2 & $c$ & 12 & 98 & [ & $\cdot$ & -2 & -2 & 4 & -2 & - ON2O \\
\hline Qㄴㄹ 21 & & $* 5 \mathrm{a}$ & 0.38 & 1.34 & 16.31 & a & * & 1 & I & c & 12 & 7.61 & $\mathrm{c}$ & $*$ & -2 & -2 & 4 & -27.0 & - ONZI \\
\hline OP 50 & BASE & $* 57$ & 53.54 & 134 & 16.00 & A & 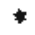 & 0 & 13 & c & 1324 & 4.52 & A & $*$ & -1 & & 4 & -20.1 & * QP 50 \\
\hline $\operatorname{ON} 22$ & & $\# 57$ & 52.61 & 134 & 11.25 & A & $*$ & 2 & 1 & C & 1449 & 981 & $c$ & $=$ & -1 & -1 & 4 & -1 & - QN22 \\
\hline $0 \times 23$ & & $\cdot 57$ & 31.50 & 136 & 9.81 & A & - & I & -1 & c & 1500 & 981 & $c$ & 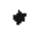 & -1 & -1 & 4 & -10 & - ON23 \\
\hline $0 \sqrt{24}$ & & * 57 & 30.38 & 134 & B. 78 & $A$ & * & 1 & -1 & $c$ & 15 & 981 & [ & - & -1 & & + & -1 & - QN24 \\
\hline Q425 & & * 57 & $49=24$ & 134 & 7.59 & a & + & 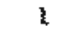 & 0 & $c$ & $*$ & 981 & $C$ & + & & & 1 & -1 & - QN2S \\
\hline ON26 & & +57 & 49.09 & 134 & 6.48 & A & $\neq$ & 2 & 0 & $c$ & 15 & 98. & c & * & -1 & & 4 & -17.4 & - ONZB \\
\hline $9 \times 27$ & & $* 57$ & 46.72 & 136 & 5.99 & A & $*$ & 2 & 0 & [ & 1546 & $98 ! 730.8 T$ & $c$ & * & -15.3 & -1 & 4 & -15.3 & - ON27 \\
\hline $\operatorname{6in} 28$ & ICHA & $\neq 57$ & 26.83 & 133 & 53.77 & 4 & * & 14 & 16 & c & 1814 & 9817 & $c$ & $*$ & -0.2 & -1 & A & -0.7 & - 9N2a \\
\hline 0129 & & $\# 57$ & 25.12 & 133 & 54.89 & 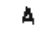 & $*$ & 4 & 6 & c & 18 & 98 & C & * & 1 & & 4 & 1.1 & - ON29 \\
\hline 0 is 30 & & $* 57$ & 26.14 & 133 & 56.43 & $A$ & * & 2 & 4 & c & 18 & 98 & C & $*$ & 2. & 2. & 4 & 2.7 & - ON3O \\
\hline QN31 & & $* 57$ & 25.01 & 131 & 57.98 & $A$ & * & 2 & 5 & c & 1857 & 981 & c & 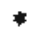 & 1. & 1. & 4 & 1.5 & * QN3I \\
\hline $0+32$ & & $* 57$ & 25.47 & 113 & 59.55 & A & $*$ & 0 & 3 & c & 1911 & 981718.43 & $c$ & * & 1.5 & 1.4 & 4 & 1.4 & - QN32 \\
\hline $9+33$ & & $* 57$ & 27.02 & 133 & 50.17 & 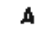 & * & 1 & 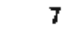 & $\mathrm{c}$ & 19 & 98172 & [ & - & 3.2 & 2.9 & 4 & 2.9 & - ON33 \\
\hline $0: 434$ & /RAN & $* 57$ & 27.78 & 133 & 59.91 & A & * & 6 & 9 & c & 1936 & 781 & 6 & - & 1. & 1.1 & 7 & 1.1 & - ON34 \\
\hline $0: 435$ & $/ 71 E$ & $* 57$ & 28.27 & 134 & 1.73 & A & - & 11 & 14 & $c$ & + & $98:$ & $c$ & * & & & 4 & & - ON35 \\
\hline 0 N36 & & -57 & 27.89 & 134 & 4.19 & $A$ & $*$ & 3 & 7 & c & * & 981718.23 & $\tau$ & $\bullet$ & -1.6 & -1.9 & 4 & -1.8 & - ON36 \\
\hline 01237 & & $* 57$ & 28.41 & 134 & 3.12 & 4 & 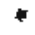 & 1 & 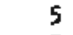 & c & 2008 & 981719.87 & $C$ & - & -0.9 & -1 & 4 & -1.0 & - ON37 \\
\hline $0: 38$ & & $* 57$ & 29.31 & 134 & 3.72 & A & * & 1 & 5 & C & 2018 & 981724.19 & c & - & & & 4 & $2 \cdot 1$ & $-0 \times 38$ \\
\hline Q 79 & TEHS & $* 57$ & 29.01 & 135 & 57.69 & $A$ & $*$ & 0 & 11 & ᄃ & 2050 & 981323.05 & a & + & 2.1 & 1.7 & 4 & 1.7 & - 9079 \\
\hline
\end{tabular}

METEP G-58 PRDBABLY HAD A +0.3 MGAL DRIFT OR TARE DURING THE AFTERNDON

DAIA_SUGYAXY

NUMGER JF STATIONS: 34

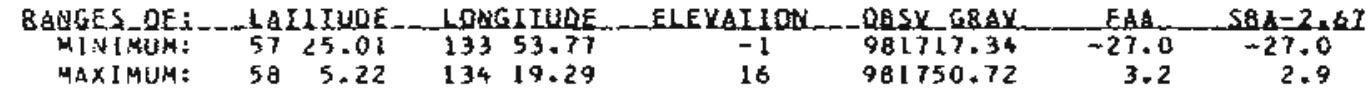




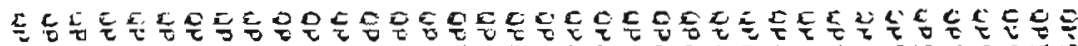

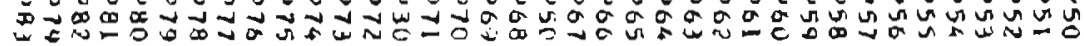

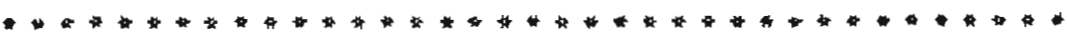

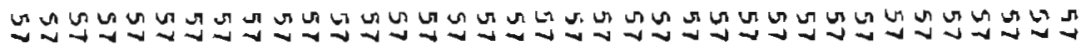

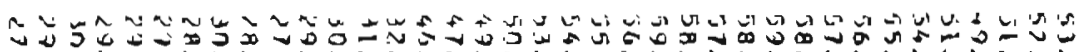

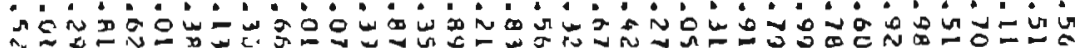

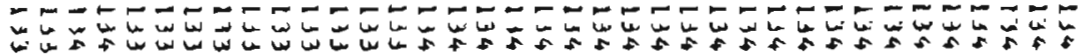
MU

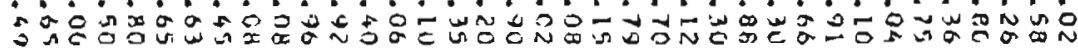

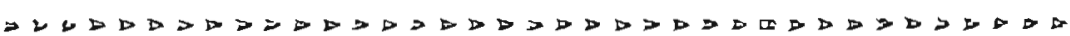

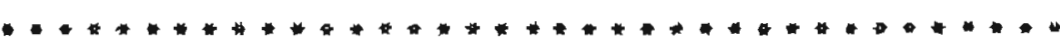

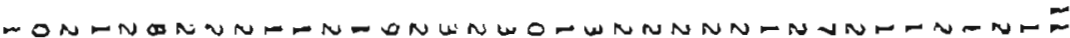

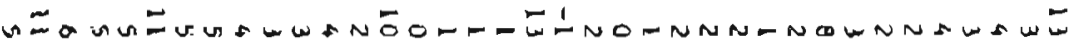

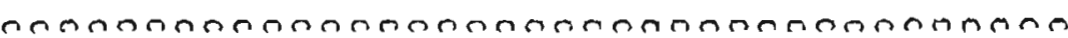

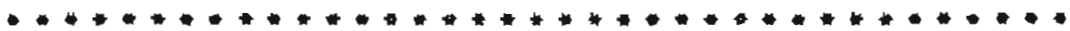

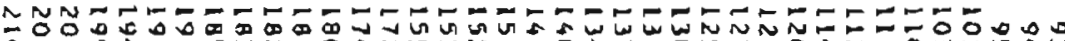

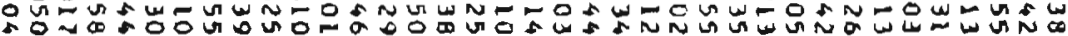

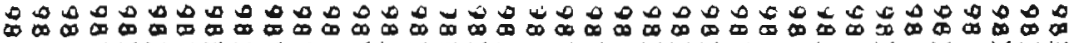

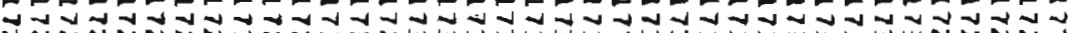
OW

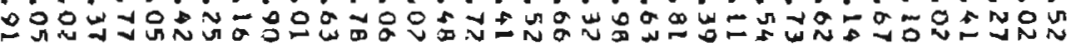

טnח

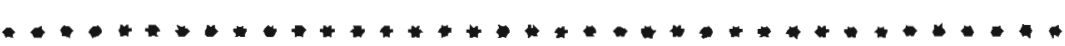

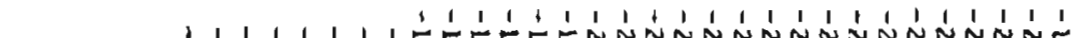
- NN N N - 更

ina un

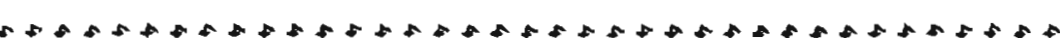

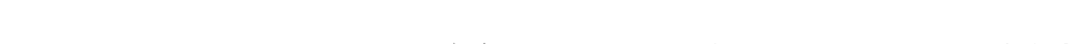

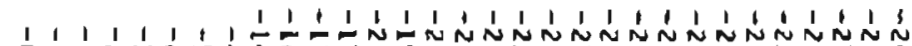

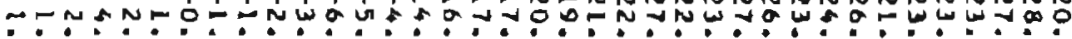
NTán-

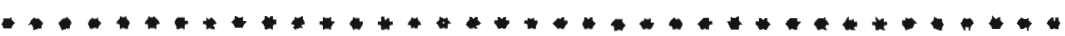

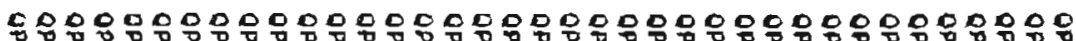

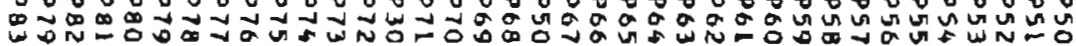



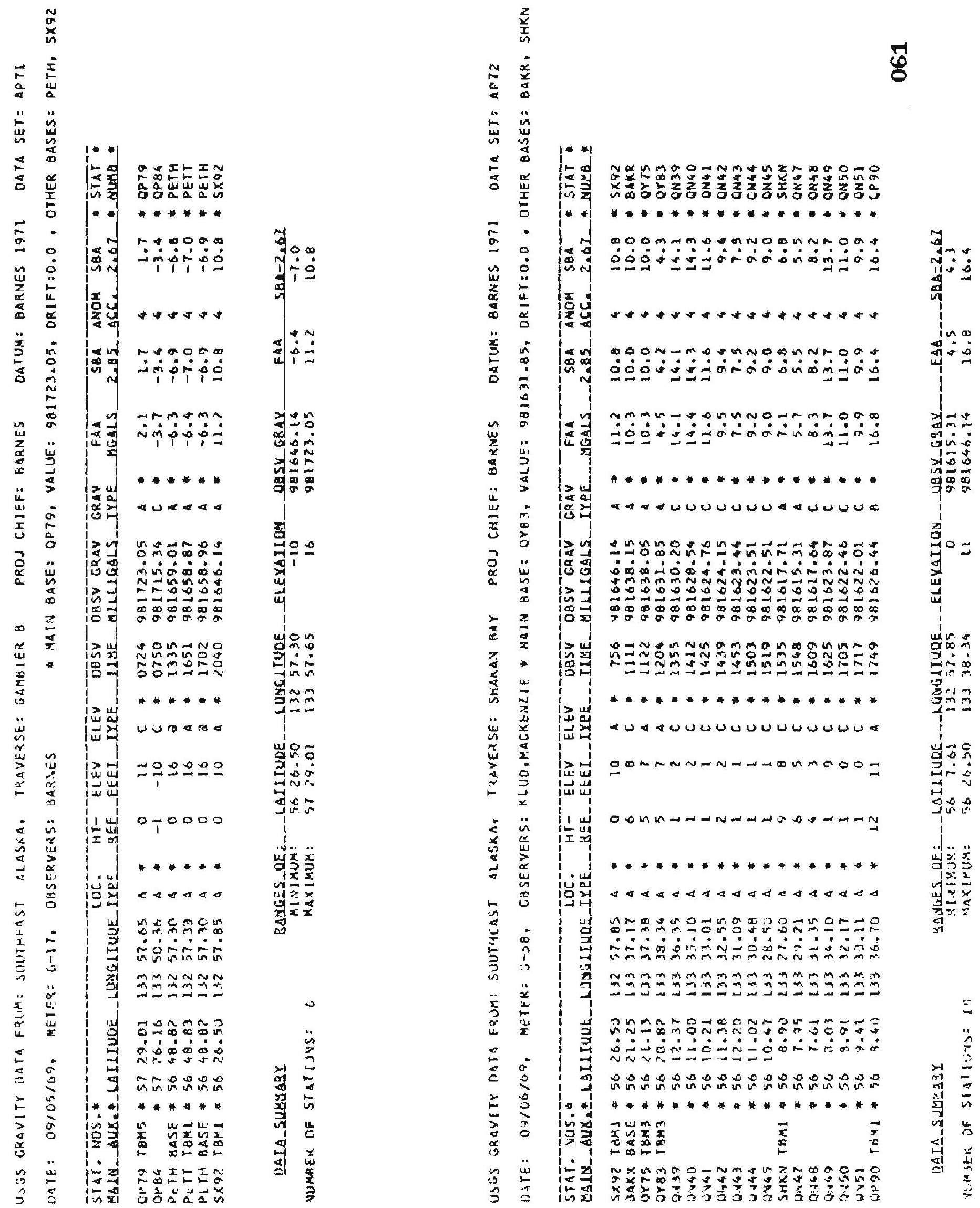


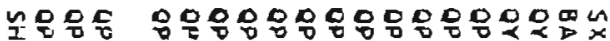

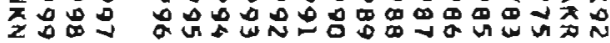

$\stackrel{\vec{\Phi}}{\underline{x}}$ $\underset{0}{2}$

$\underset{m}{\mathbf{x}}$

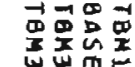

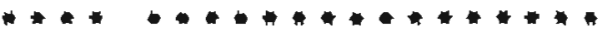

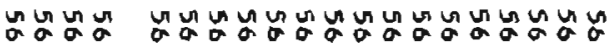

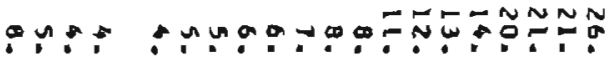

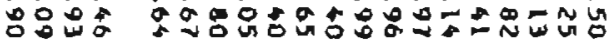

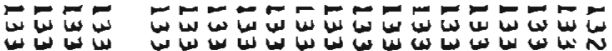

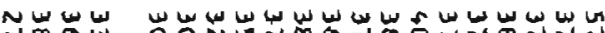

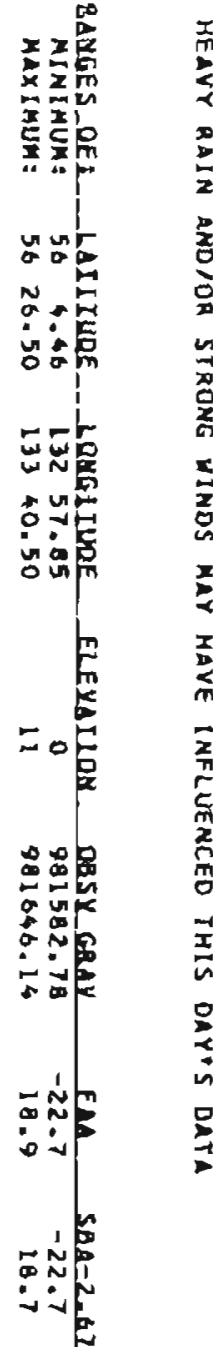

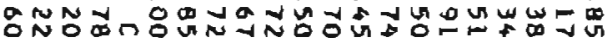
0

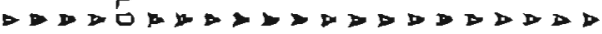

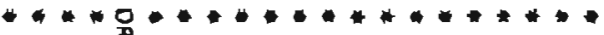

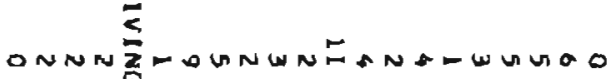

$$
\text { D }
$$

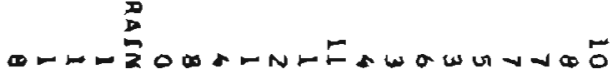

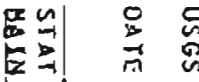

焉

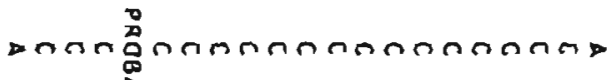
$\cdots+$ 思

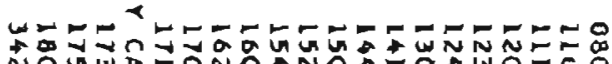
운

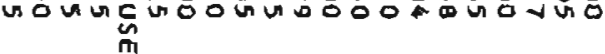

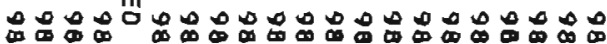

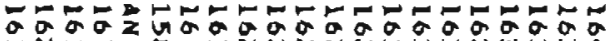
Z050

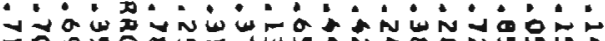

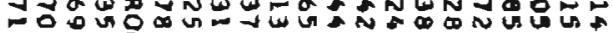

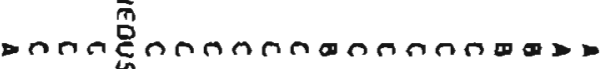

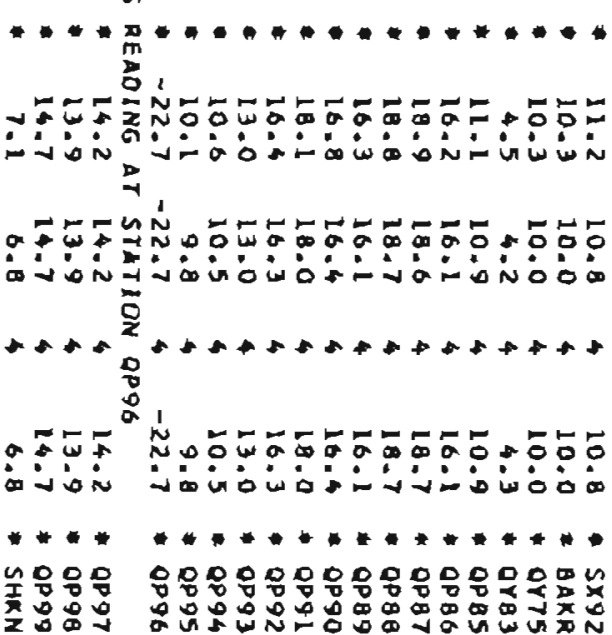




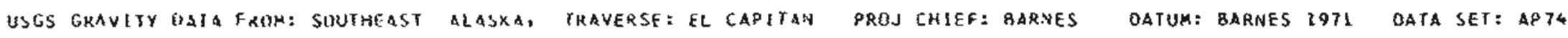

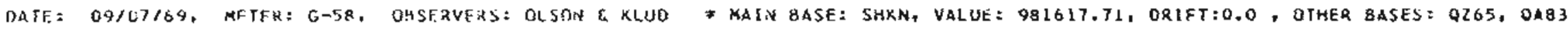

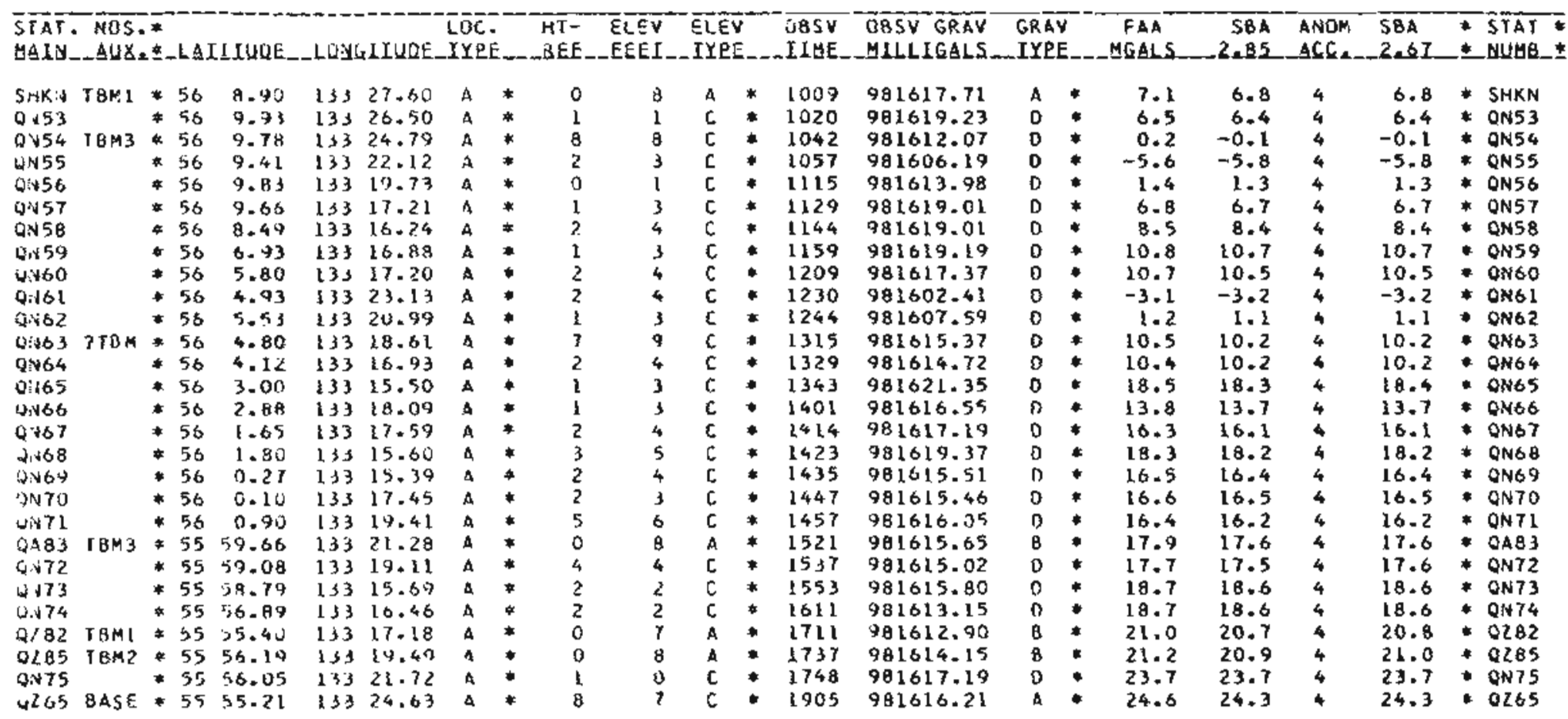

OAIA SUEMASY

WUMPER DF STAIIINS: 28

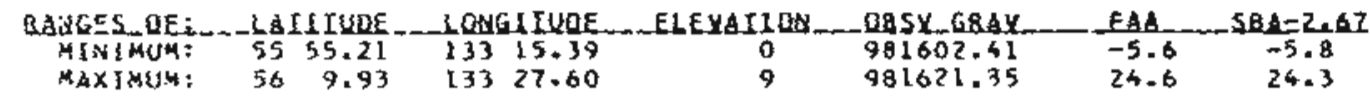




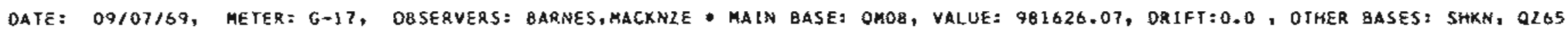

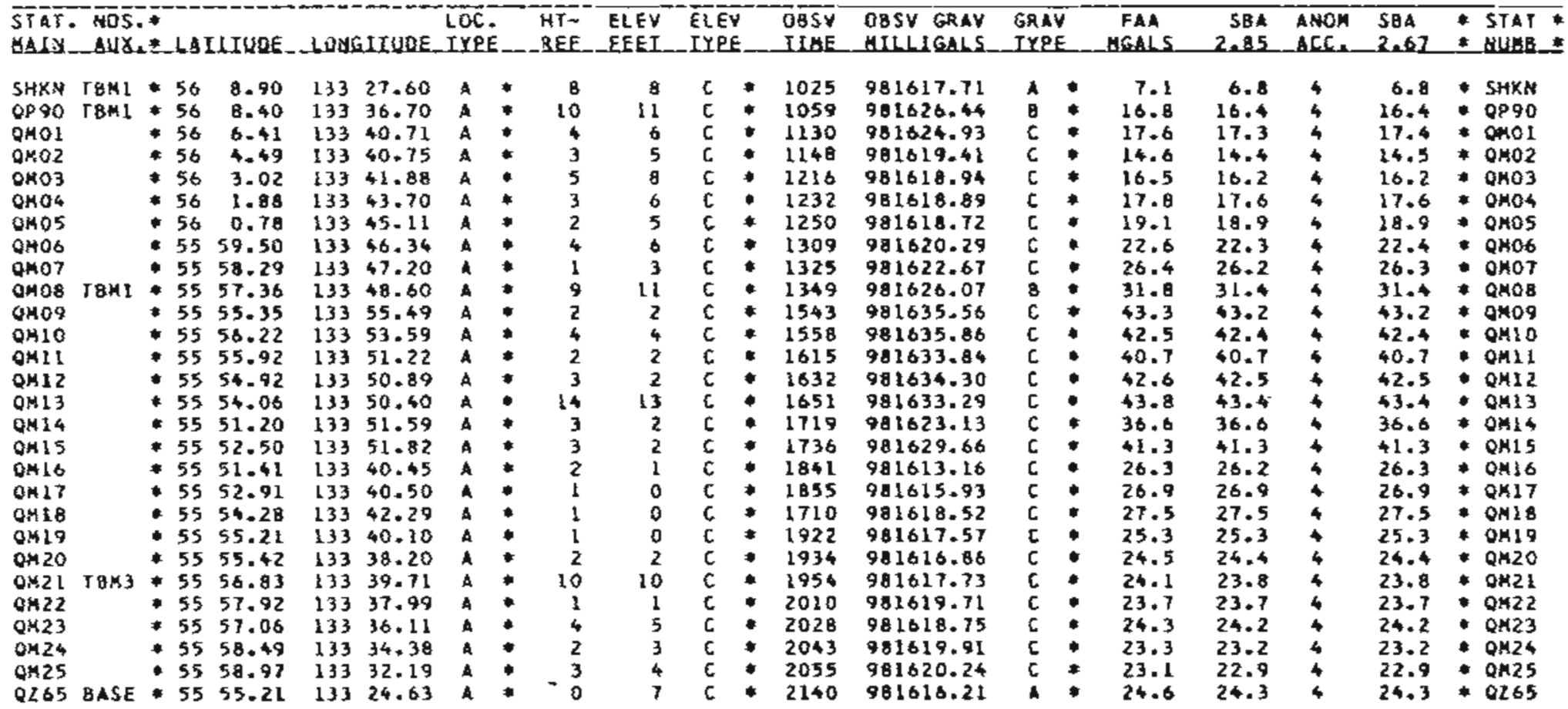

OAIA SHHEABY

NUMBER OF STATEOAS: $2 B$

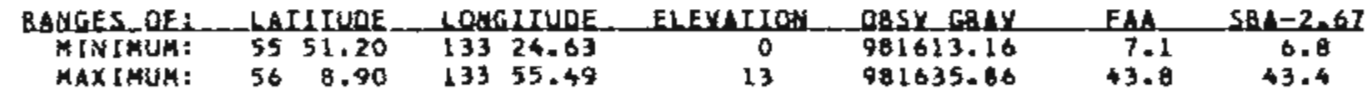


USGS GAAVITY DATA FROH: SOUTHEAST ALASXA, TRAVERSE CYRUS-AFFL PRGJ CHIEF: BARHES OATUA: BARHES I971 OATA 5ET: APTS

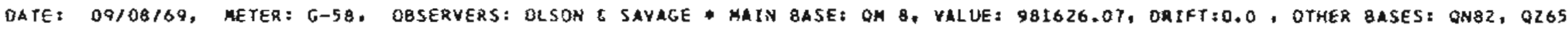

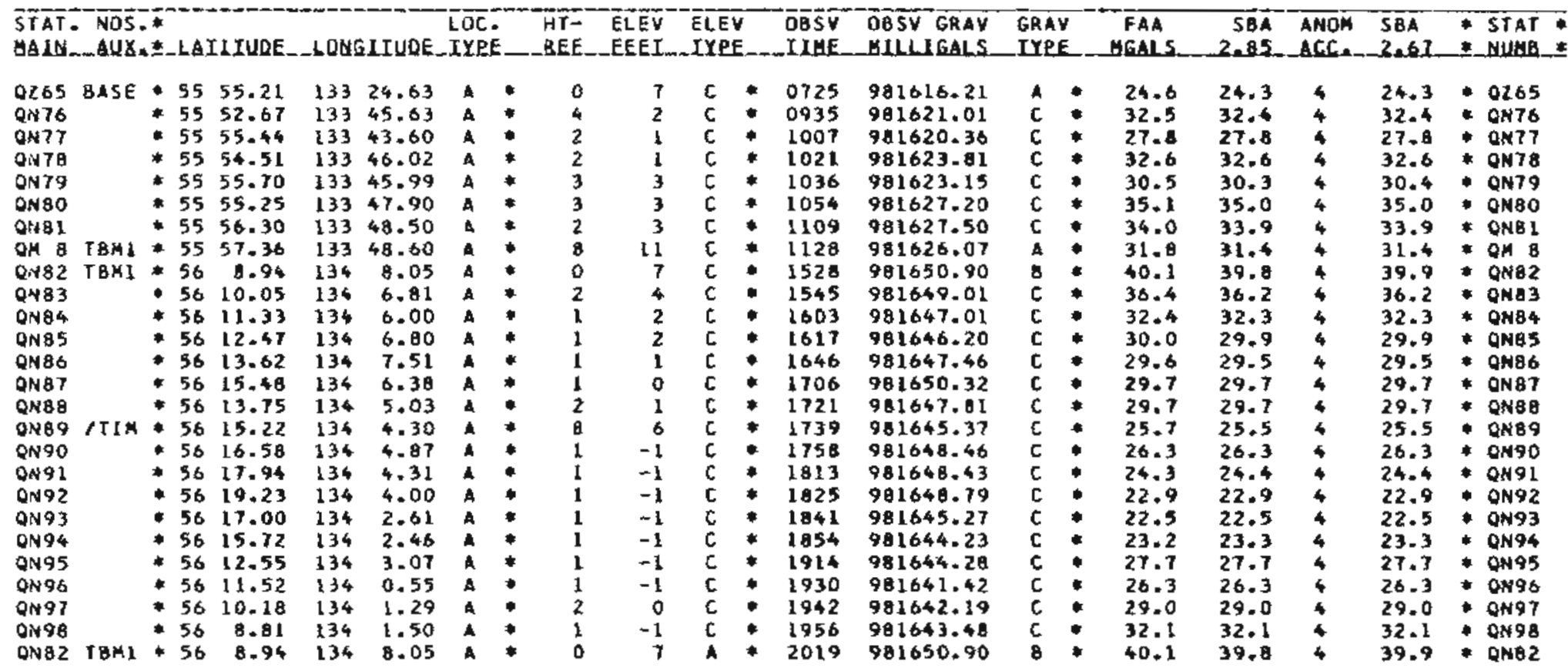

\section{QAIA_SU4SARY}

MUHBEA OF STATIOHS: 26

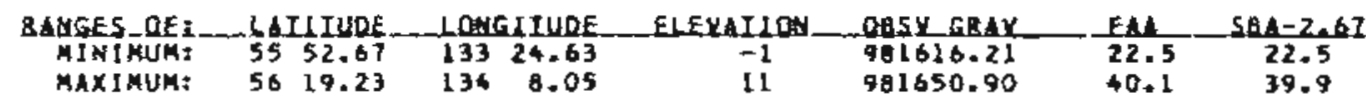


USGS GRAVITY DATA FROM: \$OJTHEAST ALASKA, TRAVERSE: AFLECK CAN

PRO」 CHIEF: BARNES

DATUH: BARNES 1971

DATA SET I APTT

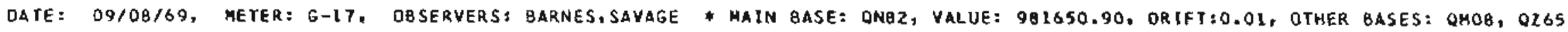

\begin{tabular}{|c|c|c|c|c|c|c|c|c|c|c|c|c|c|c|c|c|c|c|c|}
\hline 1 & & . & & & UD & & & & $\begin{array}{l}\text { EUEV } \\
\text { EEEI }\end{array}$ & $E L$ & & $\begin{array}{r}085 \% \\
\text { - ILEE. }\end{array}$ & $\begin{array}{l}\text { OBSY GRAV } \\
\text {-ULLIGALS }\end{array}$ & $\begin{array}{l}\text { GRAY } \\
\text {-IYPE }\end{array}$ & $\begin{array}{c}\text { FAA } \\
\text { HGALS }\end{array}$ & $\begin{array}{r}\text { SBA } \\
2 \mathrm{E} 5 \\
\end{array}$ & $\begin{array}{l}\text { AROH } \\
\text { ACC. }\end{array}$ & $\begin{array}{l}\text { SBA } \\
2.67\end{array}$ & $\begin{array}{l}\text { - STar } \\
\text { - MUHa }\end{array}$ \\
\hline 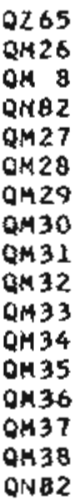 & $\begin{array}{l}\text { BASE } \\
\text { TER1 } \\
\text { TBN1 }\end{array}$ & $\begin{array}{l}* 55 \\
* 55 \\
* 55 \\
* 56 \\
* 56 \\
* 56 \\
* 56 \\
* 56 \\
* 56 \\
* 56 \\
* 56 \\
* 56 \\
* 56 \\
* 56 \\
* 56 \\
* 56\end{array}$ & $\begin{array}{r}55.21 \\
53.13 \\
57.36 \\
8.94 \\
10.11 \\
11.41 \\
10.23 \\
8.83 \\
7.41 \\
9.30 \\
6.93 \\
5.95 \\
5.00 \\
6.00 \\
7.01 \\
8.43 \\
8.94\end{array}$ & $\begin{array}{l}133 \\
133 \\
133 \\
134 \\
134 \\
134 \\
134 \\
134 \\
134 \\
134 \\
134 \\
134 \\
139 \\
134 \\
134 \\
134 \\
134\end{array}$ & $\begin{array}{r}24.63 \\
55.54 \\
48.60 \\
8.05 \\
8.70 \\
11.61 \\
11.69 \\
10.21 \\
10.37 \\
5.81 \\
6.70 \\
5.35 \\
59.79 \\
1.59 \\
2.51 \\
1.70 \\
8.05\end{array}$ & $\begin{array}{l}\mathbf{A} \\
\mathbf{A} \\
\mathbf{A} \\
\mathbf{A} \\
\mathbf{A} \\
\mathbf{A} \\
\mathrm{A} \\
\mathrm{A} \\
\mathbf{A} \\
\mathbf{A} \\
\mathbf{A} \\
\mathrm{A} \\
\mathbf{A} \\
\mathbf{A}\end{array}$ & $\begin{array}{l}* \\
* \\
* \\
* \\
* \\
* \\
* \\
* \\
* \\
* \\
*\end{array}$ & $\begin{array}{l}0 \\
1 \\
6 \\
5 \\
3 \\
1 \\
2 \\
3 \\
3 \\
3 \\
5 \\
7 \\
3 \\
3 \\
2 \\
2 \\
0\end{array}$ & $\begin{array}{r}7 \\
1 \\
11 \\
7 \\
5 \\
2 \\
2 \\
3 \\
2 \\
2 \\
3 \\
5 \\
1 \\
1 \\
0 \\
0 \\
7\end{array}$ & 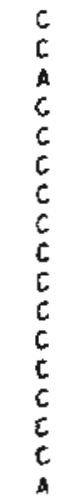 & : & $\begin{array}{l}0720 \\
1105 \\
1233 \\
1530 \\
1545 \\
1609 \\
1637 \\
1655 \\
1710 \\
1735 \\
1754 \\
1105 \\
1842 \\
1900 \\
1910 \\
1725 \\
1945\end{array}$ & $\begin{array}{l}981816.21 \\
981533.18 \\
981626.07 \\
981650.90 \\
981650.73 \\
981651.29 \\
981652.14 \\
981651.85 \\
981646.63 \\
981649.13 \\
981648.95 \\
981647.86 \\
981643.24 \\
981643.99 \\
981644.51 \\
981643.88 \\
981650.90\end{array}$ & $\begin{array}{l}1 \\
c \\
0 \\
c \\
c \\
c \\
c \\
c \\
c \\
c \\
c \\
c \\
c \\
c \\
c \\
c \\
0\end{array}$ & $\begin{array}{l}24.6 \\
43.9 \\
31.8 \\
40.1 \\
38.1 \\
36.6 \\
39.1 \\
40.8 \\
37.5 \\
38.7 \\
40.6 \\
41.0 \\
37.4 \\
36.7 \\
35.7 \\
33.1 \\
40.1\end{array}$ & $\begin{array}{l}24.3 \\
43.9 \\
31.4 \\
39.8 \\
37.9 \\
36.5 \\
39.0 \\
40.7 \\
37.4 \\
38.7 \\
40.5 \\
40.8 \\
37.3 \\
36.7 \\
35.7 \\
33.1 \\
39.8\end{array}$ & $\begin{array}{l}4 \\
4 \\
4 \\
4 \\
4 \\
4 \\
4 \\
4 \\
4 \\
4 \\
4 \\
4 \\
4 \\
4 \\
4\end{array}$ & $\begin{array}{l}24.3 \\
43.9 \\
31.4 \\
39.9 \\
37.9 \\
36.5 \\
39.0 \\
40.7 \\
37.4 \\
38.7 \\
40.5 \\
40.9 \\
37.9 \\
36.7 \\
35.7 \\
33.1 \\
39.9\end{array}$ & 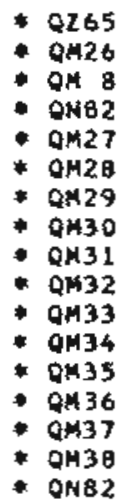 \\
\hline
\end{tabular}

DAIA_SUGHAY

NUMBER OF STATIONS: 17

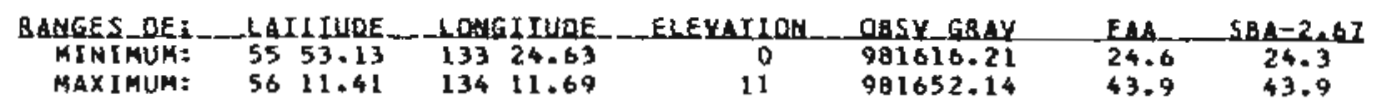


USGS GRAVITY OATA FROH SOUTHEAST ALASKA, TRAVERSE: N MALMSBAY PROJ CHIEF: GARNES DATUM: BARNES L971 DATA SEI a AP7B

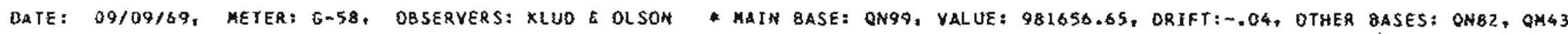

\begin{tabular}{|c|c|c|c|c|c|c|c|c|c|c|c|c|c|c|c|c|c|c|c|}
\hline $\begin{array}{l}\bar{S} \overline{F T}- \\
\text { SALE }\end{array}$ & $\begin{array}{r}\mathrm{M0} \\
-\mathrm{B}\end{array}$ & 1 & TUDE & (7) & 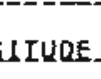 & {$[00$} & & $\overline{\mathrm{BT}}=$ & $\begin{array}{l}\text { ELEV } \\
\text { EEEI }\end{array}$ & $\begin{array}{l}\text { ELEV } \\
\text { IYEEE }\end{array}$ & $\begin{array}{l}085 V \\
\text { IIUE }\end{array}$ & $\begin{array}{l}\text { OBSV GRAV } \\
\text { HLLIGALS }\end{array}$ & $\begin{array}{l}\text { GRAY } \\
\text { IYPE }\end{array}$ & & $\begin{array}{c}\text { FAA } \\
\text { HGALS }\end{array}$ & $\begin{array}{r}584 \\
2.45\end{array}$ & $\begin{array}{l}\text { AMON } \\
\text { ACE. }\end{array}$ & $\begin{array}{l}5 \mathrm{BA} \\
2.47\end{array}$ & $\begin{array}{l}\text { STAT } \\
+ \text { SUMB }\end{array}$ \\
\hline 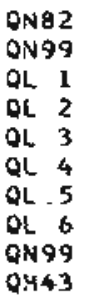 & $\begin{array}{l}\text { TEM1 } \\
\text { TEML }\end{array}$ & $\begin{array}{l}* 56 \\
* 56 \\
* 56 \\
* 56 \\
* 56 \\
* 56 \\
* 56 \\
* 56 \\
* 56 \\
* 56\end{array}$ & $\begin{array}{r}8.94 \\
18.39 \\
19.41 \\
19.60 \\
17.24 \\
17.90 \\
17.07 \\
15.90 \\
18.39 \\
25.04\end{array}$ & $\begin{array}{l}134 \\
134 \\
134 \\
134 \\
134 \\
134 \\
134 \\
134 \\
134 \\
136\end{array}$ & $\begin{array}{r}9.05 \\
14.25 \\
12.44 \\
10.01 \\
9.20 \\
10.95 \\
12.87 \\
13.65 \\
14.25 \\
1.89\end{array}$ & $\begin{array}{l}\text { A } \\
\text { A } \\
\text { A } \\
\text { A } \\
\text { A } \\
\text { A } \\
\text { A }\end{array}$ & $\begin{array}{l}* \\
* \\
* \\
* \\
* \\
* \\
*\end{array}$ & $\begin{array}{r}11 \\
3 \\
3 \\
2 \\
1 \\
2 \\
1 \\
3 \\
0 \\
19\end{array}$ & $\begin{array}{r}7 \\
7 \\
7 \\
6 \\
4 \\
5 \\
4 \\
6 \\
7 \\
10\end{array}$ & $\begin{array}{l}\mathbf{A} \\
\mathbf{A} \\
\mathbf{C} \\
\mathbf{C} \\
\mathrm{C} \\
\mathrm{C} \\
\mathrm{C} \\
\mathrm{C} \\
\mathbf{A} \\
\mathrm{A}\end{array}$ & $\begin{array}{l}0911 \\
* \quad 1354 \\
* \quad 1407 \\
+\quad 1420 \\
+\quad 1441 \\
1453 \\
+\quad 1514 \\
1532 \\
+\quad 1555 \\
1925\end{array}$ & $\begin{array}{l}981650.90 \\
981656.65 \\
901654.19 \\
901654.33 \\
981648.05 \\
981652.49 \\
981655.55 \\
981652.98 \\
981656.65 \\
981652.70\end{array}$ & $\begin{array}{l}B \\
B \\
C \\
c \\
C \\
C \\
C \\
C \\
0 \\
B\end{array}$ & $\begin{array}{l}* \\
* \\
* \\
* \\
* \\
* \\
* \\
*\end{array}$ & $\begin{array}{l}40.1 \\
32.7 \\
28.8 \\
28.6 \\
25.4 \\
29.0 \\
33.1 \\
32.4 \\
32.7 \\
19.8\end{array}$ & $\begin{array}{l}39.8 \\
32.4 \\
28.5 \\
28.4 \\
25.3 \\
28.8 \\
33.0 \\
32.2 \\
32.4 \\
19.4\end{array}$ & $\begin{array}{l}4 \\
4 \\
4 \\
4 \\
4 \\
4 \\
4 \\
4 \\
4\end{array}$ & $\begin{array}{l}39.9 \\
32.4 \\
28.6 \\
28.4 \\
25.3 \\
28.8 \\
33.0 \\
32.2 \\
32.4 \\
19.4\end{array}$ & $\begin{array}{l}\text { ONO2 } \\
\text { - QN99 } \\
\text { OL } 1 \\
\text { OL } 2 \\
\text { OL } 3 \\
\text { OL } 4 \\
\text { OL } 5 \\
\text { OL } 6 \\
\text { ON99 } \\
\text { OH43 }\end{array}$ \\
\hline
\end{tabular}

HEAUY RAIN AND/OR STROMG HINDS MAY HAVE INFLUENCED THIS DAY'S DATA

DAIA_SLUEARY

NUMGER DF STATIOAS: 10

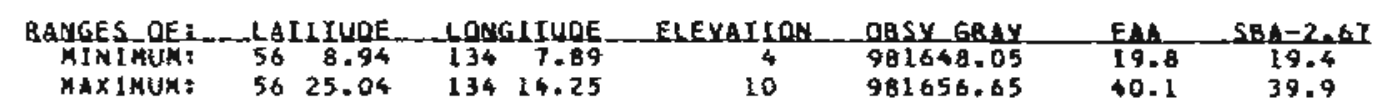

USGS GRAVITY DATA FRUM: SOUTHEAST ALASKA. TRAYERSEI S MALMSBRY PROJ CHIEFE BARNES DATUMI GARNES 197I DATA SET: APTO

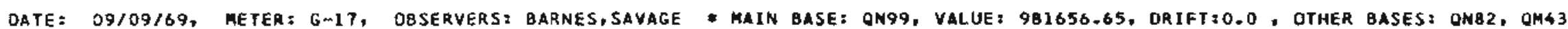

\begin{tabular}{|c|c|c|c|c|c|c|c|c|c|c|c|c|c|c|c|c|c|c|}
\hline †4 & & & 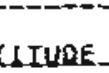 & & & LOC. & & $\begin{array}{l}\text { MT- } \\
\text { CREE }\end{array}$ & $\begin{array}{l}\text { ELEY } \\
\text { EEEI }\end{array}$ & $\begin{array}{l}\text { ELEV } \\
\text { IYPE }\end{array}$ & $\begin{array}{l}\text { Dasy } \\
\text { IlleE }\end{array}$ & $\begin{array}{l}\text { DOSV GRAV } \\
\text { OLLIGLS }\end{array}$ & $\begin{array}{l}\text { GRAY } \\
\text { IYPE }\end{array}$ & $\begin{array}{c}\text { Fin } \\
\text { MGLS }\end{array}$ & $\begin{array}{r}584 \\
2.15 \\
\end{array}$ & $\begin{array}{l}\text { Mon } \\
\text { Act. }\end{array}$ & $\begin{array}{l}584 \\
2,42\end{array}$ & 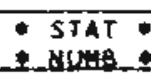 \\
\hline $\begin{array}{l}482 \\
499 \\
2439 \\
440 \\
441 \\
442 \\
443\end{array}$ & $\begin{array}{l}8 \times 1 \\
8 \times 1\end{array}$ & 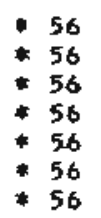 & $\begin{array}{r}7.94 \\
18.39 \\
17.23 \\
16.02 \\
15.00 \\
14.35 \\
25.04\end{array}$ & $\begin{array}{l}134 \\
134 \\
134 \\
134 \\
134 \\
134 \\
134\end{array}$ & $\begin{array}{r}9.05 \\
14.25 \\
16.85 \\
16.10 \\
13.98 \\
12.35 \\
7.89\end{array}$ & $\begin{array}{l}A \\
A \\
A \\
A \\
A \\
A \\
A\end{array}$ & : & $\begin{array}{r}12 \\
3 \\
2 \\
2 \\
1 \\
1 \\
13\end{array}$ & $\begin{array}{r}7 \\
7 \\
6 \\
5 \\
4 \\
4 \\
10\end{array}$ & $\begin{array}{l}c \\
c \\
c \\
c \\
c \\
c \\
c\end{array}$ & $\begin{array}{l}0905 \\
1355 \\
1425 \\
1440 \\
1510 \\
1525 \\
1920\end{array}$ & $\begin{array}{l}981650.90 \\
901656.65 \\
981657.65 \\
901655.28 \\
981650.91 \\
981649.79 \\
981652.70\end{array}$ & 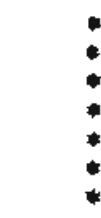 & $\begin{array}{l}32.7 \\
35.2 \\
34.4 \\
31.4 \\
31.2 \\
19.8\end{array}$ & & $\begin{array}{l}0 \\
0 \\
0 \\
0 \\
0\end{array}$ & $\begin{array}{l}39.9 \\
32.4 \\
35.0 \\
34.3 \\
31.2 \\
31.0 \\
19.4\end{array}$ & 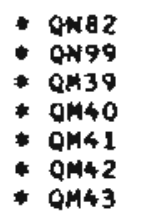 \\
\hline
\end{tabular}

HEAVY RAIN ALD/GR STRONG WIMOS MAY HAVE IHFLUENCEO THIS DAY"S OATA

TATA_SLHBSA

NUMBEA DF STATIONS: 7

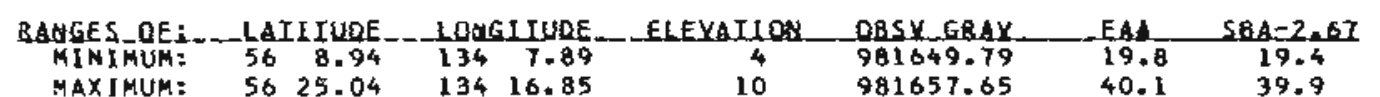




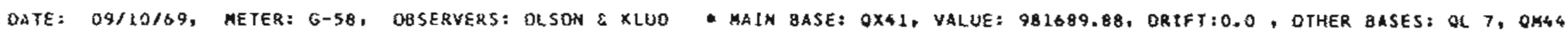

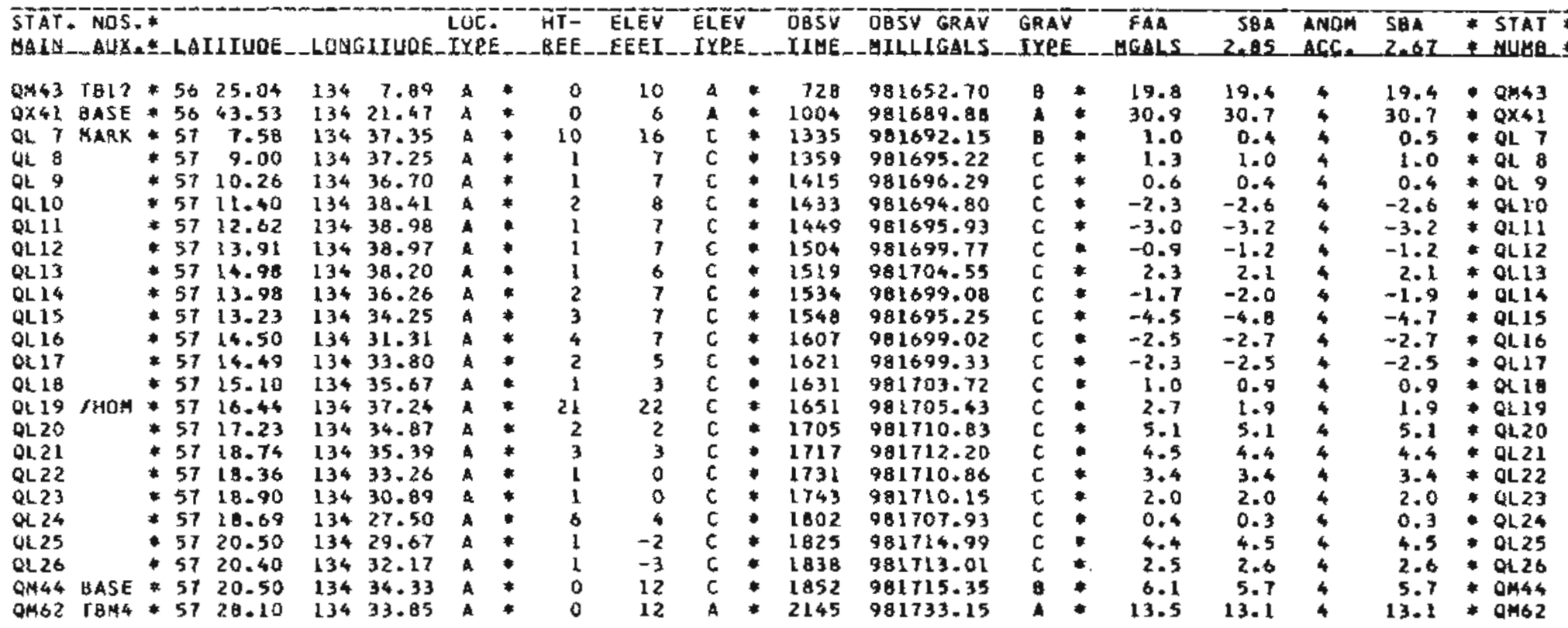

OAIA SHBHagr

NUMBER OF STATIONSI 24

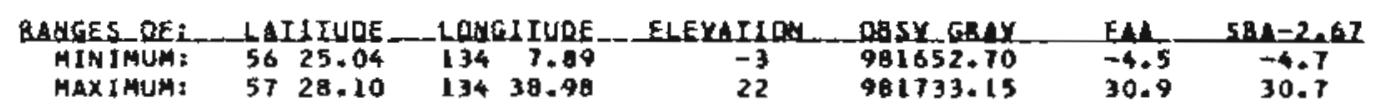




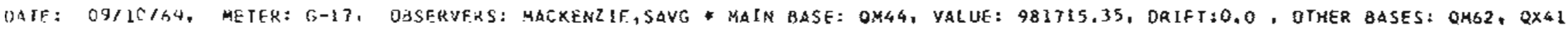

\begin{tabular}{|c|c|c|c|c|c|c|c|c|c|c|c|c|c|c|c|c|c|c|c|c|c|}
\hline $\begin{array}{l}\text { STAR } \\
\text { MALIV }\end{array}$ & $\begin{array}{l}\text { Nos } \\
\text { SuYS }\end{array}$ & & & Uus & & ILID & 1 & & $1=$ & $\begin{array}{l}\overline{L E V} \\
\overrightarrow{E E} E\end{array}$ & $\begin{array}{l}\text { EL } \\
\text { IY }\end{array}$ & & $\begin{array}{l}\text { UBSV } \\
\text { ILUE. }\end{array}$ & $\begin{array}{l}\text { DESY GAAY } \\
\text { ULLLGALS }\end{array}$ & $\bar{G} \bar{P}$ & & GGALS & $\begin{array}{r}58 A \\
2.85\end{array}$ & & $\begin{array}{l}564 \\
2.61\end{array}$ & $\begin{array}{l}57 \text { AT } \\
\text { *NUHB }\end{array}$ \\
\hline $0 M 43$ & JBL? & * & 56 & 25.04 & 134 & $7 . \mathrm{AO}$ & A & * & 0 & 10 & A & 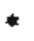 & 320 & 981652.70 & $B$ & * & 19.8 & 19.4 & 4 & 19.4 & + 0.443 \\
\hline $9 x+1$ & d $45 F$. & . & 56 & 47.53 & 234 & $21.4 \pi$ & A & 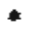 & 10 & 6 & C & * & 1005 & 981689.88 & A & * & 30.9 & 30.7 & 4 & 30.7 & $=0 \times 41$ \\
\hline Q.444 & & t. & 57 & $20=50$ & 134 & $34 \cdot 33$ & A & 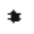 & 6 & 12 & $c$ & - & 1516 & 781715.35 & 8 & * & 6.1 & 5.7 & 4 & 5.7 & - OMn44 \\
\hline $0 \times 45$ & & * & 57 & 21.90 & 154 & 34.05 & $\Delta$ & * & 2 & 6 & c & * & 1602 & 981715.44 & c & + & 3.7 & 3.5 & 4 & 3.5 & -01445 \\
\hline $\sin 46$ & & $\%$ & 53 & 23.32 & 134 & 34.27 & A & * & 2 & 5 & C & * & 1625 & $9: 1722.34$ & $c$ & * & $\theta .6$ & 8.4 & 4 & 6.4 & - Q446 \\
\hline $\operatorname{Jim} 47$ & & * & 57 & 26.44 & 134 & 33.30 & 4 & * & 2 & 4 & $c$ & - & 1645 & 981722.30 & $\varepsilon$ & - & $6 \cdot 8$ & 6.7 & 4 & 6.7 & * QM4T \\
\hline $9 \times 49$ & & * & 57 & 23.34 & 134 & 31.00 & A & * & 1 & 2 & C & * & 1709 & 981720.96 & c & $*$ & 6.8 & 6.8 & 4 & 6.8 & - OHAB \\
\hline DM 49 & & * & 57 & 27.75 & 134 & 29.82 & A & * & 1 & 2 & c & $*$ & 1724 & 981717.17 & $\mathrm{C}$ & * & 3.9 & $3 . a$ & 4 & 3.8 & * QM49 \\
\hline OM 50 & & * & 57 & $22=26$ & 134 & 27.30 & A & $*$ & 1 & $\mathbf{l}$ & c & * & $1 \geqslant 40$ & 981713.84 & $c$ & * & 1.1 & 1.1 & 4 & 1.1 & - 0.450 \\
\hline ans 1 & & * & 57 & 22.30 & 134 & 24.55 & A & * & 1 & 0 & $c$ & * & 1755 & 981713.42 & c & * & 0.0 & 0.6 & 4 & 0.6 & - OM51 \\
\hline Ges2 & & $*$ & 57 & 20.47 & 134 & 21.32 & A & * & 2 & 0 & c & * & 1日 13 & 981710.87 & $c$ & * & 0.5 & 0.5 & 4 & 0.5 & - Q452 \\
\hline UN53 & & $\neq$ & 57 & 20.31 & 154 & 19.40 & a & 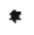 & 3 & 0 & C & * & 1832 & 981709.58 & [ & * & -0.5 & -0.5 & 4 & -0.5 & - OMS3 \\
\hline $0+54$ & & $*$ & 5.7 & 21.59 & 134 & 22.21 & A & $*$ & 3 & 0 & $c$ & * & 1848 & 991709.85 & $\mathrm{c}$ & $*$ & $-2 \cdot 0$ & -2.0 & 4 & -2.0 & - QM54 \\
\hline Q455 & & * & 57 & 23.07 & 134 & 22.05 & A & $\neq$ & 5 & 1 & C & - & 1909 & 991713.31 & c & - & -0.5 & -0.5 & 4 & -0.5 & - Q455 \\
\hline g45o & & * & 57 & 27.68 & 134 & 20.39 & 4 & & 1 & -4 & $\mathrm{c}$ & * & 1922 & 981714.93 & c & * & -0.2 & -0.0 & 4 & -0.0 & - QM56 \\
\hline QM 57 & & * & 51 & $23+0 u$ & 134 & 25.54 & 4. & $*$ & 1 & -4 & E & * & 1945 & 981712.75 & c & $\bullet$ & $-1 \cdot 5$ & -1.4 & 4 & -1.4 & - QMST \\
\hline J45B & & * & 57 & 2.3 .62 & 154 & 27.99 & 4 & * & 1 & -5 & C & * & 2005 & 981712.96 & $c$ & - & -2.2 & -2.0 & 4 & $-2,0$ & - oks8 \\
\hline dis 57 & & * & 51 & 24.87 & 134 & 29.46 & $\mathrm{~A}$ & 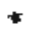 & I & -5 & c & * & 2022 & 981718.43 & $c$ & * & 1.7 & 1.9 & 4 & 1.0 & - 0458 \\
\hline $9 \times 64$ & & - & 57 & 25.65 & 136 & 32.02 & $A$ & * & 5 & 0 & c & * & 2037 & 981724.53 & $c$ & * & 7.1 & $t-i$ & 4 & 7.3 & - 9460 \\
\hline UMG & & $\#$ & 57 & 77.06 & 134 & 32.35 & A & $*$ & 2 & -3 & C & - & 2054 & 981729.12 & C & $*$ & 9.5 & 9.6 & 4 & 9.6 & - 0461 \\
\hline QM6? & IBM4 & * & 57 & 20.10 & 134 & 33.85 & A & * & 16 & 12 & A & 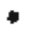 & 2120 & 981733.15 & A & $\star$ & 13.5 & {$[3.1$} & 4 & 13.1 & $+0 M 62$ \\
\hline
\end{tabular}

vaIa_SUMugY

WUAHE OF STATLUNS: 26

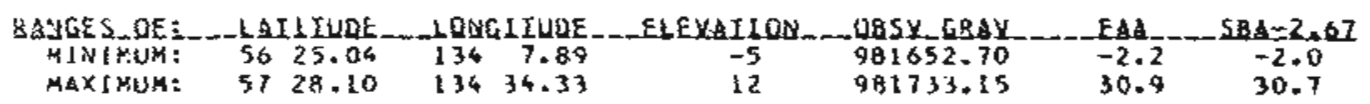


USGS GRAVITY DATA FROK: 5OUTHEAST ALASXA, TRAVERSE F KOOTZNAHOO

PROJ EHIEF: QARES

DATUM: GARNES 1971

DATA SET: APB 3

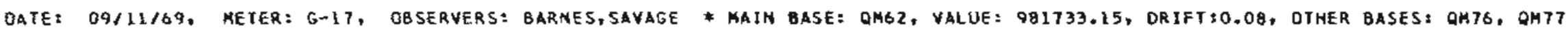

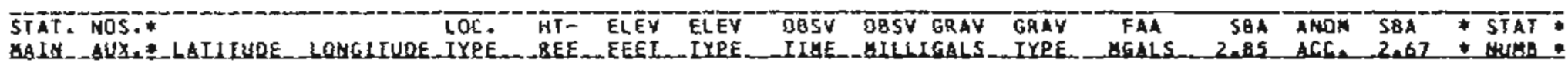

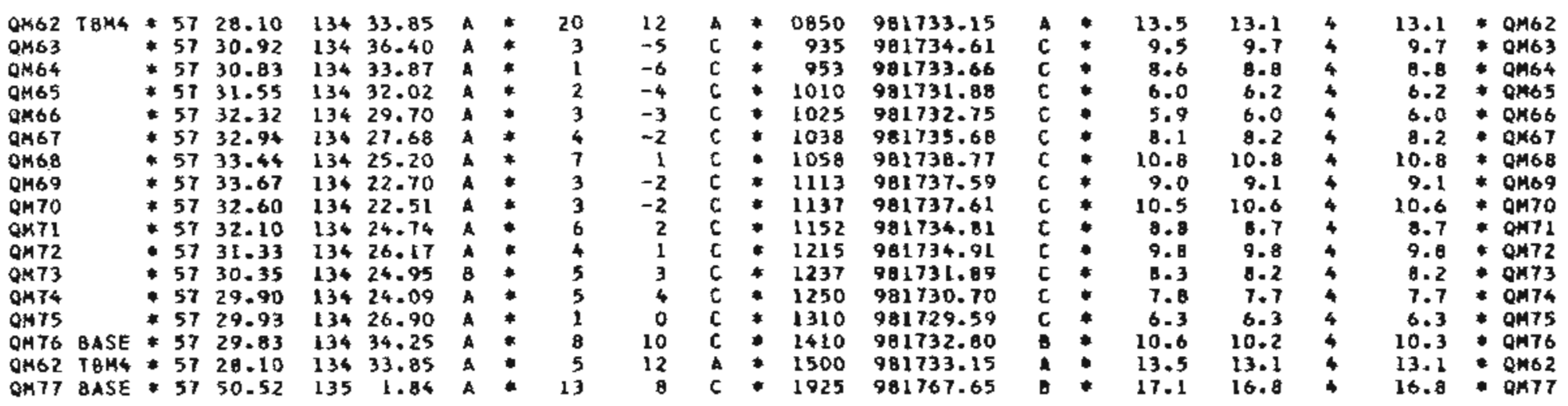

DaI A SuUtaBY

HUHBER OF STATIONS: 17

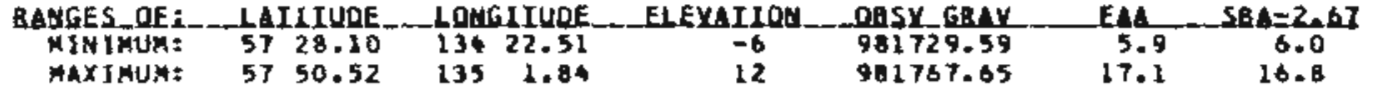




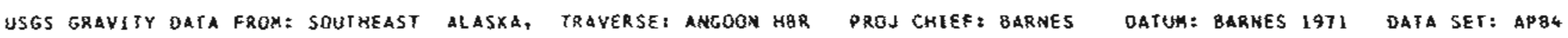

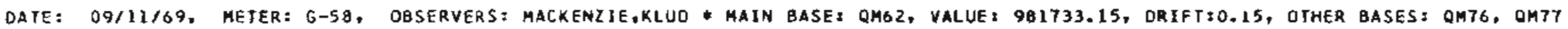

\begin{tabular}{|c|c|c|c|c|c|c|c|c|c|c|c|c|c|c|c|c|c|c|c|}
\hline TAIE & 14 & & & & cup & & & I- & ELEY & & & $\begin{array}{l}\text { ORSY } \\
\text { ILME }\end{array}$ & $\begin{array}{l}\text { DBSY GRAV } \\
\text { ULLLIGALS }\end{array}$ & YAPE & $\begin{array}{l}\text { FAA } \\
\text { - GGALS }\end{array}$ & $\begin{array}{r}581 \\
2.05\end{array}$ & ACO & $\begin{array}{l}584 \\
2,67\end{array}$ & $\begin{array}{l}\text { STAT } \\
\text { SNULA_F }\end{array}$ \\
\hline 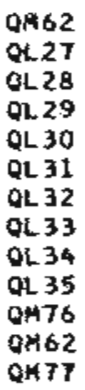 & $\begin{array}{l}\text { BASE } \\
\text { TBM4 } \\
\text { BASE }\end{array}$ & $\begin{array}{l}57 \\
* 57 \\
* 57 \\
* 57 \\
* 57 \\
* 57 \\
+57 \\
+57 \\
+57 \\
* 57 \\
* 57 \\
+57 \\
57\end{array}$ & $\begin{array}{l}28.10 \\
28.54 \\
29.75 \\
28.11 \\
29.03 \\
30.74 \\
30.73 \\
31.90 \\
30.86 \\
29.98 \\
29.83 \\
28.10 \\
50.52\end{array}$ & $\begin{array}{l}134 \\
134 \\
134 \\
134 \\
134 \\
134 \\
134 \\
134 \\
134 \\
134 \\
134 \\
134 \\
135\end{array}$ & $\begin{array}{r}33.85 \\
36.10 \\
35.10 \\
30.57 \\
32.50 \\
32.71 \\
30.70 \\
28.70 \\
28.56 \\
27.82 \\
34.25 \\
33.05 \\
1.84\end{array}$ & $\begin{array}{l}A \\
A \\
A \\
A \\
A \\
A \\
A \\
A \\
A \\
A \\
A\end{array}$ & $\begin{array}{l}* \\
* \\
* \\
* \\
*\end{array}$ & $\begin{array}{l}0 \\
1 \\
1 \\
1 \\
2 \\
1 \\
2 \\
2 \\
3 \\
2 \\
6 \\
5 \\
13\end{array}$ & $\begin{array}{r}12 \\
-7 \\
-6 \\
-5 \\
-3 \\
-2 \\
0 \\
-2 \\
0 \\
0 \\
14 \\
12 \\
8\end{array}$ & $\begin{array}{l}A \\
E \\
C \\
C \\
E \\
C \\
C \\
C \\
C \\
C \\
C \\
A \\
C\end{array}$ & * & $\begin{array}{l}0055 \\
0925 \\
0953 \\
1030 \\
1040 \\
1103 \\
1115 \\
1145 \\
1225 \\
1246 \\
1414 \\
1502 \\
1932\end{array}$ & $\begin{array}{l}981733.15 \\
981734.57 \\
981733.62 \\
981726.04 \\
981730.79 \\
981732.16 \\
981728.81 \\
941720.99 \\
981723.40 \\
981728.19 \\
981732.80 \\
981733.15 \\
981767.65\end{array}$ & $\begin{array}{l}A \\
C \\
C \\
C \\
C \\
C \\
C \\
C \\
C \\
C \\
D \\
A\end{array}$ & $\begin{array}{r}13.5 \\
12.5 \\
10.0 \\
5.6 \\
8.5 \\
7.6 \\
4.4 \\
-5.2 \\
-1.1 \\
4.8 \\
11.0 \\
13.5 \\
17.1\end{array}$ & $\begin{array}{r}13.1 \\
12.8 \\
10.2 \\
5.8 \\
8.6 \\
7.7 \\
4.4 \\
-5.1 \\
-1.1 \\
4.8 \\
10.5 \\
13.1 \\
16.0\end{array}$ & $\begin{array}{l}4 \\
4 \\
4 \\
4 \\
4 \\
4 \\
4 \\
4 \\
4 \\
4 \\
4\end{array}$ & $\begin{array}{r}13.1 \\
12.8 \\
10.2 \\
5.8 \\
0.6 \\
7.7 \\
4.1 \\
-5.1 \\
-1.1 \\
4.8 \\
10.5 \\
13.1 \\
16.8\end{array}$ & 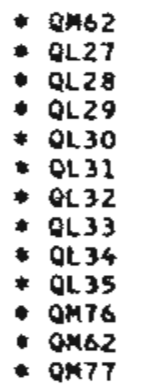 \\
\hline
\end{tabular}

\section{DAIA_sugBa8Y}

NUMAER DF STATIOHSI I3

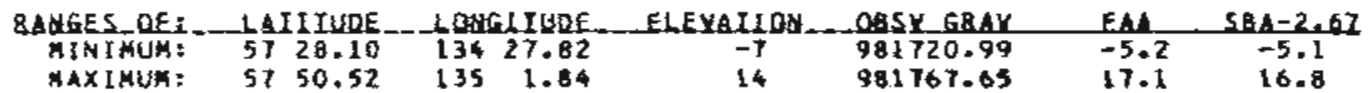


空

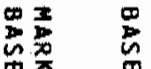

******************

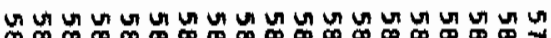

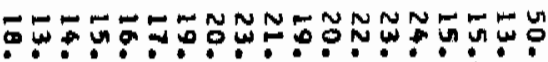

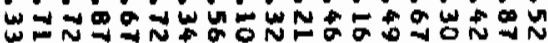

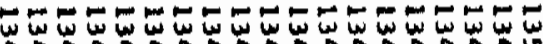

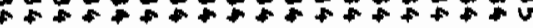

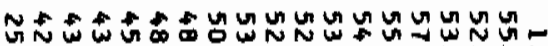

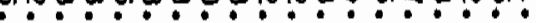

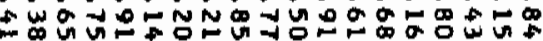

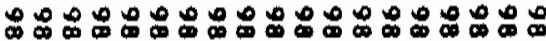

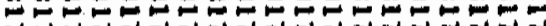

ज讨

U:NONON

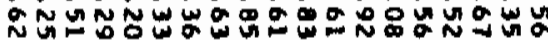

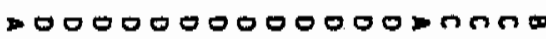

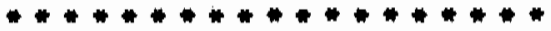

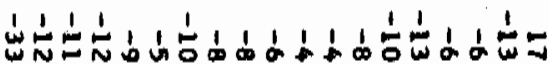
舟?

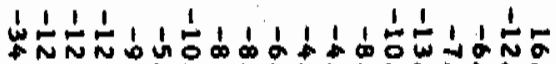

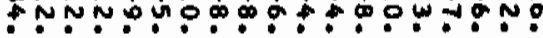

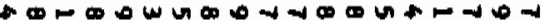

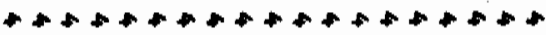

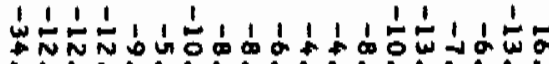

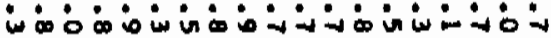

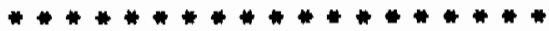

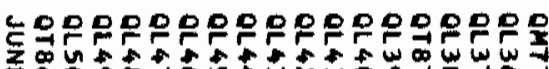




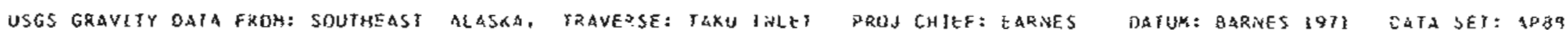

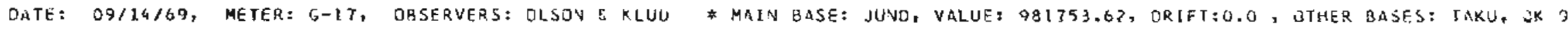

\begin{tabular}{|c|c|c|c|c|c|c|c|c|c|c|c|c|c|c|c|c|c|c|}
\hline $\begin{array}{l}5 T A Y \\
\text { YALS }\end{array}$ & NO & & & & 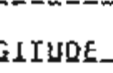 & & & $1-$ & & & $\begin{array}{l}\mathrm{BSV} \\
\mathrm{LEE}\end{array}$ & $\begin{array}{l}\text { UQSW GRAV } \\
\text { SLLLGALS }\end{array}$ & IY & FAA & $\begin{array}{r}50 \\
285\end{array}$ & $\mathrm{MUt}$ & $\begin{array}{l}S B A \\
2.47\end{array}$ & $\begin{array}{r}5 T A T \\
\text { HUSB }\end{array}$ \\
\hline JUND & BA5E & 59 & 18.33 & 134 & 25.41 & A & \# & 0 & 17 & $\varepsilon$ & 913 & 981753.62 & $A$ & -33.7 & -34.4 & 4 & -34.3 & * JUV 0 \\
\hline Mo5 & & 48 & 13.73 & 134 & 15.70 & A & 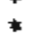 & 2 & -6 & 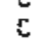 & 1027 & 796.04 & 0 & -2 & & 4 & & - OHB5 \\
\hline 1486 & /ENT & - 58 & 2.65 & 134 & 13.80 & $A$ & . & 22 & 14 & c & 1045 & 981753.39 & D & -26.6 & -27.1 & 4 & -27.1 & - OHé \\
\hline 407 & & +5B & & 134 & & A & * & I & -6 & $E$ & 1052 & 981753.51 & $\mathrm{D}$ & -27.9 & -2 & $\because$ & & - Q4.487 \\
\hline QM 89 & & - $5 \mathrm{a}$ & {$[2,51$} & 134 & $1-20$ & $a$ & 4 & 3 & -4 & $c$ & 1101 & 481754.86 & i) & -26.6 & $-2 b+5$ & 4 & -26.5 & - Gtos \\
\hline $0 \div 89$ & & +58 & 12.37 & 134 & 10.04 & A & $*$ & 2 & -5 & $c$ & $\log$ & 301753,24 & $\mathrm{n}$ & $-28,2$ & -2 & 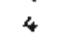 & $-2 \pi .0$ & - Gasey \\
\hline 0490 & & - 58 & 12.20 & 134 & 8.73 & A & - & 1 & -5 & $\mathrm{c}$ & 1120 & y. & n & $-3 i$ & -3 & 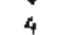 & .5 & 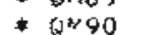 \\
\hline QM91 & & $* 50$ & 13.02 & 134 & 8.49 & A & * & 1 & -5 & C & $\operatorname{ll} 30$ & 42,81 & D & -39.5 & -3 & 4 & -39.3 & - 0MO1 \\
\hline DN 92 & & $* 58$ & 33 & 134 & 3 & $A$ & $*$ & $i$ & -5 & $c$ & $1] 38$ & $9 B 1+42.02$ & 0 & -41.1 & -48 & $\therefore$ & .0 & - 0.492 \\
\hline $0+93$ & & * 58 & 13 & 13.4 & 7.1 & A & * & i & -4 & c & 1147 & 981 & T? & -41.9 & -41.8 & s & $-4 i, 9$ & - OM93 \\
\hline 0494 & & * 50 & $14+27$ & 134 & 6.03 & $A$ & \# & $i$ & -3 & $t$ & . & 4.06 & D & -39.7 & -39.6 & 4 & -39.6 & - OM94 \\
\hline 0495 & & - 58 & 24. & 134 & 0 & a & * & $i$ & -3 & $c$ & 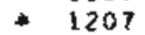 & 42.07 & $\mathrm{p}$ & -42.5 & -4 & 4 & .4 & - Q405 \\
\hline omgs & & * 58 & 15.52 & L34 & 6.80 & $A$ & $*$ & $\mathrm{i}$ & -2 & $c$ & 1218 & 35.83 & D & $-49 \cdot 5$ & -49.5 & 4 & -4 & * 01448 \\
\hline 0497 & & +58 & 16.41 & 134 & & A & - & i & -1 & $c$ & 1231 & 98 & 1) & -41 & -4 & 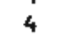 & -4 & - OMэ7 \\
\hline $0 \times 98$ & & -50 & 7 & 34 & 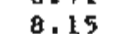 & 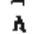 & 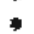 & 1 & 0 & c & - 1245 & 981 & 0 & -51.4 & -5 & 4 & .4 & - 0498 \\
\hline 0499 & $1 C A 5$ & - 58 & 18.84 & 134 & 0.10 & A & $*$ & L 8 & 18 & $\bar{c}$ & * & 98 & $n$ & -5 & -5 & 4 & -5 & - 04999 \\
\hline QK 1 & & +50 & 19.80 & 134 & $3+94$ & 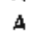 & * & 1 & 1 & 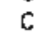 & $*$ & & $\Gamma$ & -6 & & 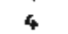 & & - Qk 1 \\
\hline $0 \times 2$ & & +58 & $21.2 k$ & 136 & 3.35 & $A$ & * & $i$ & 2 & $\mathrm{c}$ & 1327 & 98 & 0 & -7 & -7 & 4 & -7 & $+0 \times 2$ \\
\hline OK 3 & & * 58 & 22.78 & 114 & 3.33 & $A$ & - & $i$ & 3 & $\varepsilon$ & 1344 & 981710.74 & n & -83.9 & -8 & 4 & -84.0 & $+0 \times 1$ \\
\hline OK 4 & & $\neq 58$ & 24.22 & 194 & 2.49 & A & * & I & 4 & C & 1403 & 98170 & n] & -8 & -35 & 4 & & * OK 4 \\
\hline & & -58 & & 134 & 0.04 & $A$ & * & 4 & 8 & $c$ & + & B & 0 & -9 & -1 & 4 & & $-4 x$ \\
\hline $0 \times 6$ & & - 58 & 25.97 & 133 & 58.91 & A & - & 2 & 7 & $c$ & 1436 & 301708.58 & $\Rightarrow$ & -90.0 & -4 & 4 & -4 & 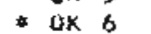 \\
\hline & & $\neq 58$ & & 133 & & A & * & $?$ & a & c & 1450 & 981 & D & & -8 & 4 & & + wK 7 \\
\hline UK $A$ & & - $5 \theta$ & 28.22 & 133 & 50.61 & A & * & $t$ & $\mathrm{~A}$ & c & 1507 & 991714.33 & 0 & $-a$ & -87.5 & 4 & $-\mathrm{t}$ & $-0 k 8$ \\
\hline ox $\$$ & & $=58$ & 29.49 & 133 & 56.40 & A & 6 & 31 & 39 & c & 1535 & $981308-17$ & a & -92.2 & -95.6 & 4 & .5 & $=0 \times 9$ \\
\hline T\&KU & BASF. & -58 & $\angle 9.51$ & 433 & 56.35 & A & $\cdot$ & 29 & 38 & c & 1637 & 981703.21 & $\mathrm{~B}$ & -42.2 & -93.6 & 4 & $=43.5$ & * TAKU \\
\hline
\end{tabular}

QAIA_SUEMASY

HUHBER DF STATIONS: 26

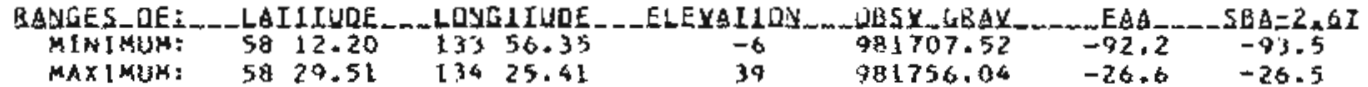




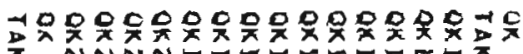

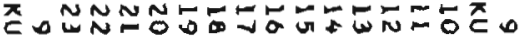

罗品

总怘

罢品

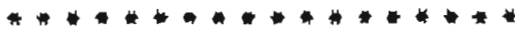

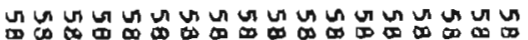

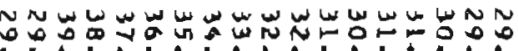

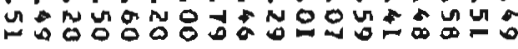

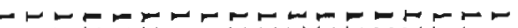

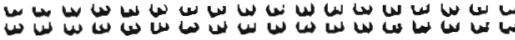

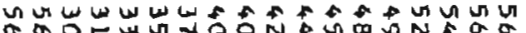

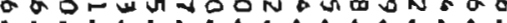

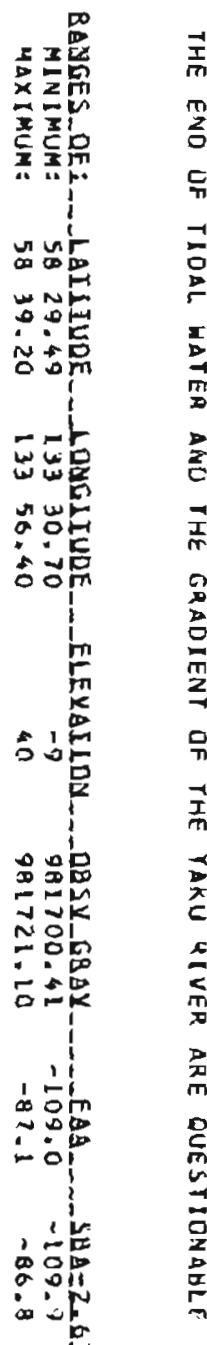

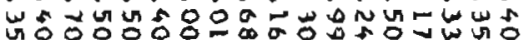

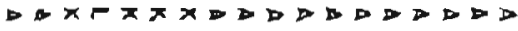

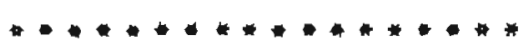

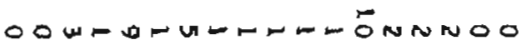

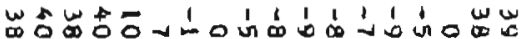

necectectedectinn

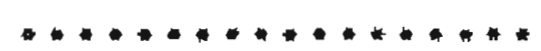

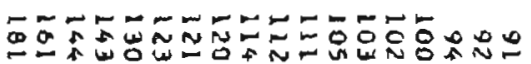

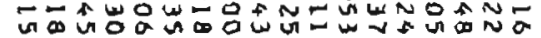

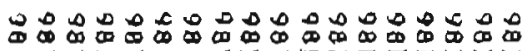

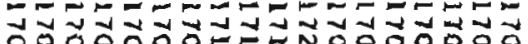

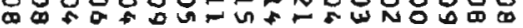

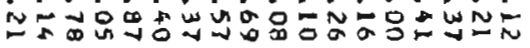

$\therefore \infty 0000030000000000$

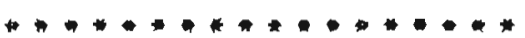

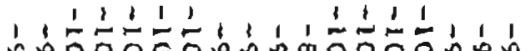

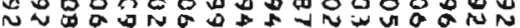

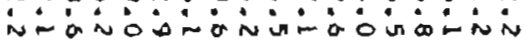

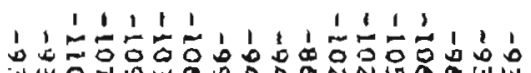

w。

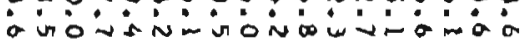

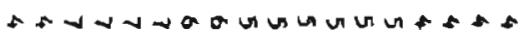

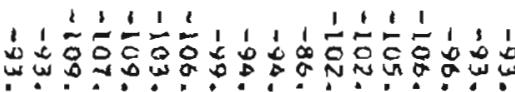

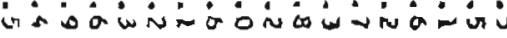

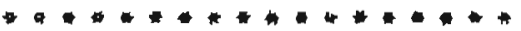

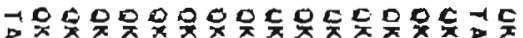

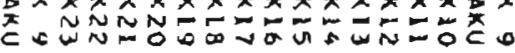

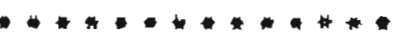

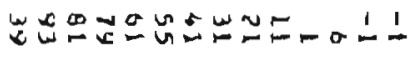

nocosedockonon

ง

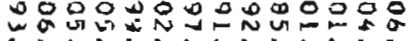

u

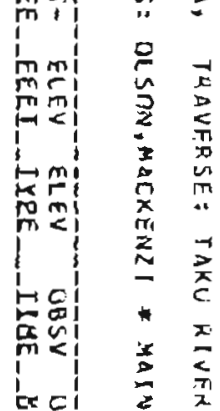

票 
USGS GRAVITY DATA FROM: SOUTHEAST ALASXA, TRAVERSE = TAXU SOUTH

PROJ CHIEFI BARNES

DATUH: BARNES 1971

OATA SET: AP 90

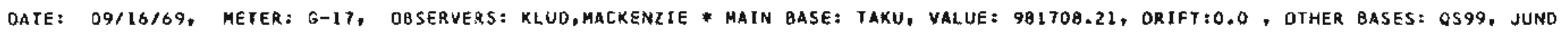

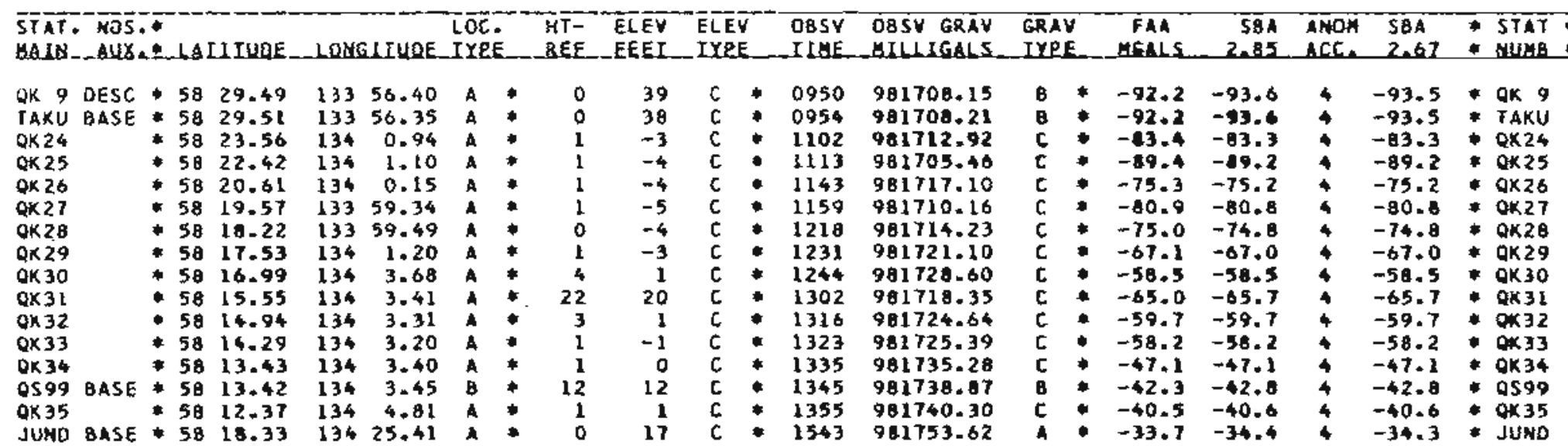

RAIA_SLHGABY

NUMBER DF STATIONS: 16

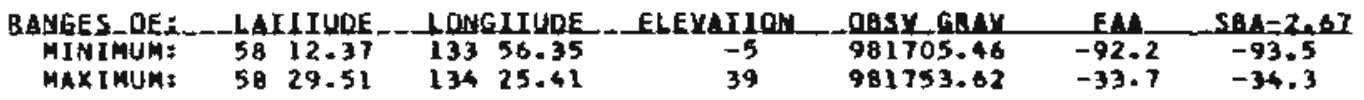

